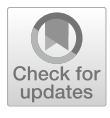

Cite as

Nano-Micro Lett.

(2022) 14:68

Received: 14 November 2021 Accepted: 14 January 2022 Published online: 26 February 2022 (C) The Author(s) 2022

\section{State of the Art and Prospects in Metal-Organic Framework-Derived Microwave Absorption Materials}

\author{
Shuning Ren ${ }^{1}$, Haojie $\mathrm{Yu}^{1}{ }^{凶}$, Li Wang $^{1}$, Zhikun Huang ${ }^{1}$, Tengfei Lin ${ }^{1}$, Yudi Huang ${ }^{1}$, \\ Jian Yang ${ }^{1}$, Yichuan Hong ${ }^{1}$, Jinyi Liu ${ }^{1}$
}

\title{
HIGHLIGHTS
}

- The metal organic frameworks derived microwave absorption materials (MOF derived MAMs) were systematically reviewed.

- The design principles, preparation methods and effect of microstructures and composites of MOF derived MAMs were discussed.

- The challenges and further research directions of MOF derived MAMs were presented

ABSTRACT Microwave has been widely used in many fields, including communication, medical treatment and military industry; however, the corresponding generated radiations have been novel hazardous sources of pollution threating human's daily life. Therefore, designing high-performance microwave absorption materials (MAMs) has become an indispensable requirement. Recently, metal-organic frameworks (MOFs) have been considered as one of the most ideal precursor candidates of MAMs because of their tunable structure, high porosity and large specific surface area. Usually, MOF-derived MAMs exhibit excellent electrical conductivity, good magnetism and sufficient defects and interfaces, providing obvious merits in both impedance matching and microwave loss. In this review, the recent research progresses on MOF-derived MAMs were profoundly reviewed, including the categories of MOFs and MOF composites precursors, design principles, preparation methods and the relationship between mechanisms

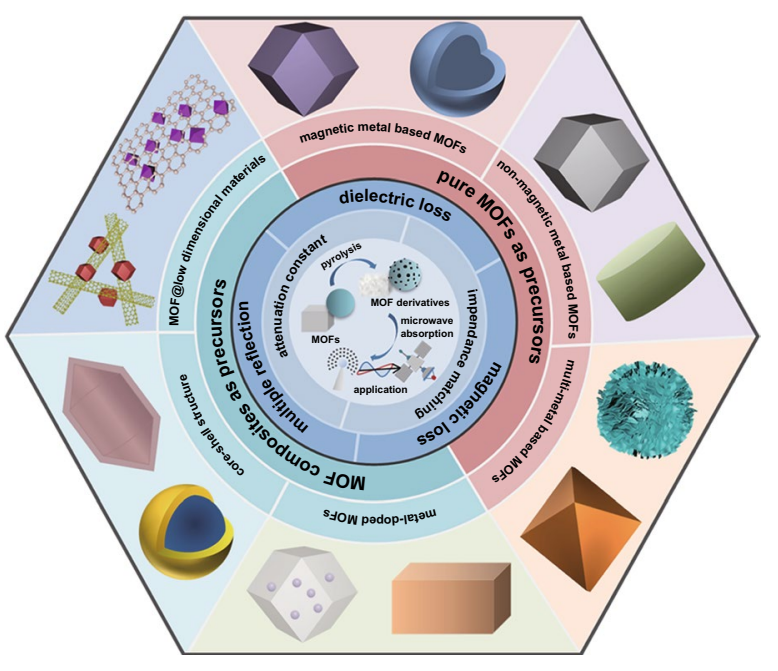
of microwave absorption and microstructures of MAMs. Finally, the current challenges and prospects for future opportunities of MOFderived MAMs are also discussed.

KEYWORDS Microwave absorption materials; Metal-organic frameworks; Preparation methods; Mechanisms of microwave absorption

1 State Key Laboratory of Chemical Engineering, College of Chemical and Biological Engineering, Zhejiang University, Hangzhou 310027, People's Republic of China 


\section{Introduction}

Microwave technology has been utilized in both civil and military fields, such as wireless communication, medical treatment, scientific research and especially military industry, which are related to national security and social development [1, 2]. Nevertheless, the signals of precision instruments are seriously affected by surrounding microwave radiations [3-5]. Moreover, the excessive microwave emission has been considered as a novel hazardous source of pollution deeply, significantly threating human health $[6,7]$. Therefore, designing high-performance microwave absorption materials (MAMs) has become an urgent and indispensable demand [8-10]. Usually, the incident microwave on the MAM surface will be divided into three parts: absorbed, reflected and transmitted waves. To shield microwaves as much as possible, the ideal MAMs should possess good absorption, wide absorption range, light weight and thin thickness. In past decades, many MAMs were designed and investigated, which can be roughly containing three categories: conductive polymers [10-13] (such as polyaniline (PAIN) and polypyrrole (PPy)), conductive carbon matrices [14-16] (such as grapheme (GO) and carbon nanotube (CNT)) and magnetic materials $[17,18]$ (such as $\mathrm{Fe}_{3} \mathrm{O}_{4}$ and barium ferrite). Although they have many advantages such as cheap, easily available and simple preparation, the mismatched impedance caused by single component severely limited their practical application. The combination of conductive materials and magnetic materials has been proved as an effective method to solve above issue [19], whereas the traditional preparation methods such as electrochemical plating [20], ball-milling [21, 22] and impregnation [23] are only processed at molecular level, which cannot get the MAMs with uniformly component distribution and high porosity. Therefore, designing MAMs with excellent microwave absorption (MA) performance using the effective method is highly desired.

Metal-organic frameworks (MOFs), consisting of the metal ions/cluster centers and organic ligands through coordination interactions, exhibit unique merits including high porosity, large specific surface area and tunable structure [24-26], enabling them huge promising application in various areas such as heterogeneous catalysts[27], energy storage and conversion [28, 29], gas separation [30, 31], sensing [32, 33] and biomedicine [34]. In 2015, the first MOF-derived MAM sample was reported by Liu and coworkers [35]. The ZIF-67 was selected as a precursor to obtain porous $\mathrm{Co} / \mathrm{C}$ nanocomposites after heat treatment; the prepared $\mathrm{Co} / \mathrm{C}$ nanocomposites exhibited favorable $\mathrm{MA}$ performance. Since then, many MOF-derived MAMs with (a)

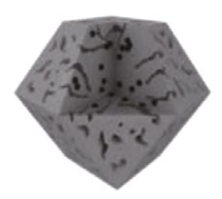

(b)

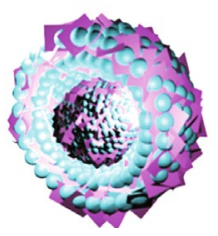

(d)

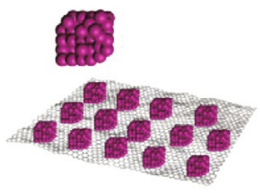

porous

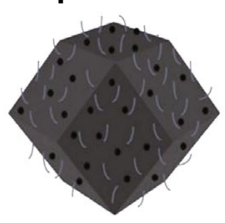

core-shell

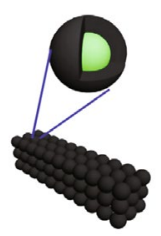

electric network

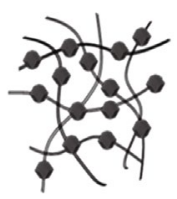

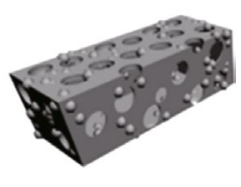

(c) hollow
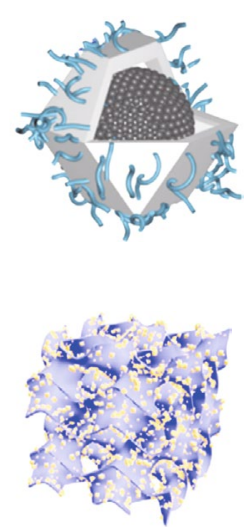

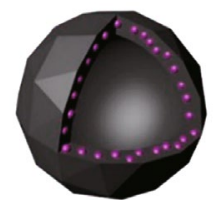

(e) multilayer

\section{(c) hollow}
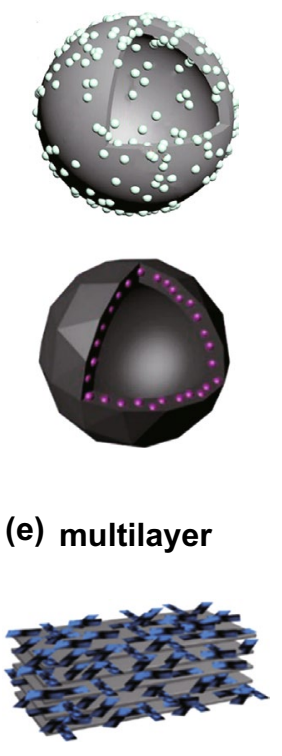

Fig. 1 MOF-derived MAMs with different microstructures: a porous structure, b core-shell structure, $\mathbf{c}$ hollow structure, d electric network, and e multilayer structure. Reprinted with permission from Refs. [36-47] 


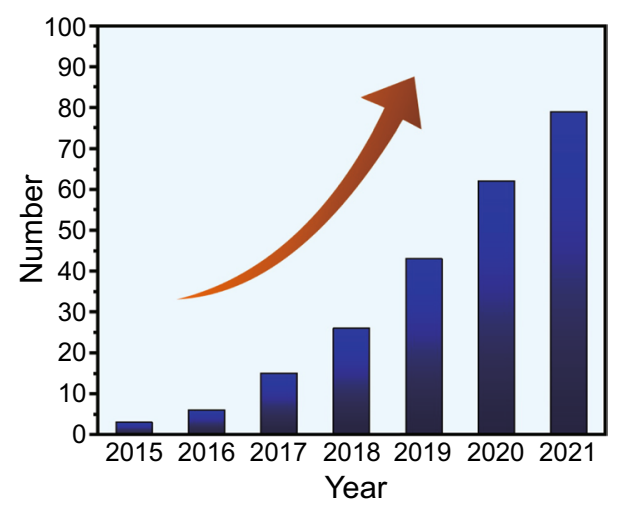

Fig. 2 Number of published papers of MOF-based MAMs from 2015 to 2021(Web of science)

various microstructures have been designed and reported (Fig. 1) [36-47]. Compared with other microwave absorbers, MOF-derived MAMs can maintain the original porous framework and uniform pore distribution and can obtain enhanced magnetic property after appropriate high temperature pyrolysis, promoting the improvement of MA performance [48-50].

Previous works suggested that the pyrolysis temperature, atmosphere and soaking time can have the effect on the composition and structure of MAMs [47, 51]. The pyrolysis temperature mainly affects the graphitization degree of carbon matrix and microstructure of MAMs. The graphitization degree is positively correlated with pyrolysis temperature; higher graphitization degree can provide greater electrical conductivity, which is in favor of the conductivity loss to incident microwave. However, excessive conductivity has negative effects on the MA properties, which can stimulate the induced current when microwave incident was on the surface of MAMs, leading to the enhancement of the microwave reflection and weakening the absorption, resulting in worse MA properties, which is called "skin effect." On the other hand, surplus conductivity can interact with the incident microwave to form an induced magnetic field in the high-frequency magnetic field, resulting in magnetic energy radiation and negative magnetic loss. Besides, ultrahigh pyrolysis temperature might lead to the collapse of $\mathrm{MOF}$ skeletons, resulting in the destruction of porous structures, which is bad for multiple reflection and electromagnetic synergy. For instance, Wang used ZIF-67 with hierarchically porous structure as sacrificial template to obtain porous carbon matrix at three temperatures of 500,700 , and $900{ }^{\circ} \mathrm{C}$ [37]. The results showed that the higher temperature could improve the crystallinity, graphitization degree and magnetism of carbon matrix, while the structure was collapsed. Besides, the chemical reaction during the carbonization and chemical constitution of MAMs are also influenced by the pyrolysis atmosphere including argon gas (Ar), nitrogen gas $\left(\mathrm{N}_{2}\right)$, air and mixed gas $\left(\mathrm{Ar} / \mathrm{H}_{2}, \mathrm{~N}_{2} / \mathrm{H}_{2}\right)$. Ar and $\mathrm{N}_{2}$ provide the stable condition for the formation of metal nanoparticles. In the case of preparing metal oxides, high-temperature heat (a)

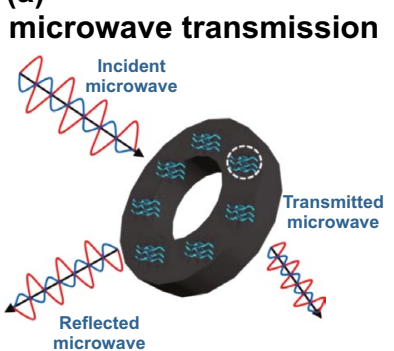

(b)

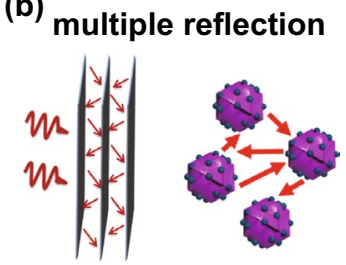

(c)
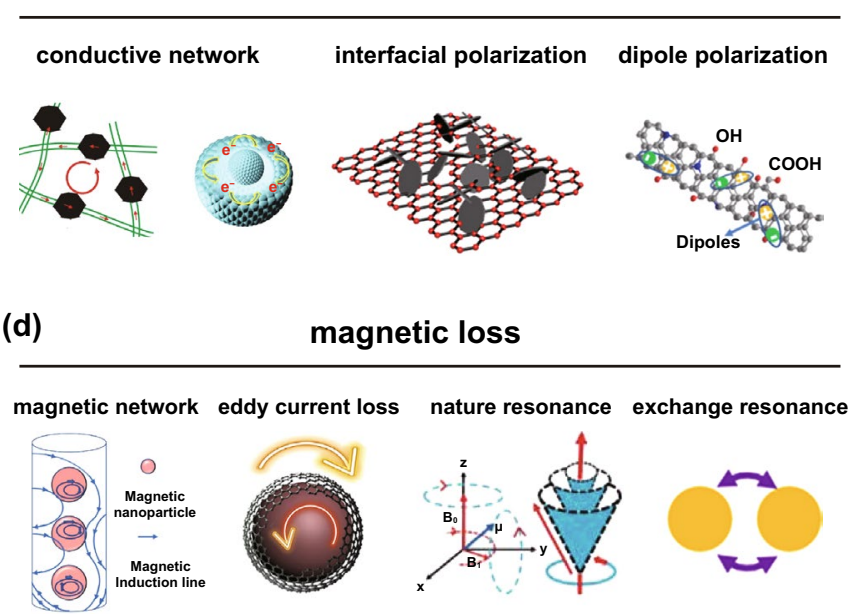

Fig. 3 a Illustration of the microwave absorption mechanism, including microwave transmission path, b multiple reflection inside the MAMs, $\mathbf{c}$ dielectric loss (conductive network, interfacial polarization and dipole polarization) and $\mathbf{d}$ magnetic loss (magnetic coupling network, eddy current loss, nature resonance and exchange resonance). Reprinted with permission from Refs. [40, 59-68] 
treatment is usually carried out directly in air. Besides, the thermal decomposition rate of the material in air is higher than that in $\mathrm{Ar}$ and $\mathrm{N}_{2}$. As a reducing gas, $\mathrm{H}_{2}$ could promote the reduction of metal ions to metal nanoparticles. Especially in the presence of transition metals, the graphitization of carbon can be accelerated effectively, so that $\mathrm{Ar} / \mathrm{H}_{2}$ and $\mathrm{N}_{2} / \mathrm{H}_{2}$ are also commonly used in some pyrolysis processes.

MOF-derived MAMs have attracted tremendous research attentions, especially in the last three years, over 150 papers about MOF-derived MAMs have been published (Fig. 2). However, compared with other MAMs materials such as polymer nanocomposites [52, 53], graphene foams [54, 55] and MXenes [56-58], the investigation of MOF-derived MAMs was still in its infant stage; the intrinsic principles of MOFderived MAMs were still rarely summarized and reviewed. Herein, the recent progress on MOF-derived MAMs is profoundly reviewed. In the initial part of this review, the mechanism of microwaves absorption is introduced. Then, according to the magnetic property of mental center/cluster and number of metallic elements in each center note of MOFs, the MA performance of various MAMs derived from bare MOFs and MOF composites is systematically analyzed to deeply understand the relationship between internal mechanisms and microstructure of MAMs. Moreover, the influences of some crucial parameters in the synthesis of MOF and high-temperature pyrolysis on the whole MA performance are also discussed. In the end, the current challenges are pointed out, and future prospects of MOF-derived MAMs are also given.

\section{Mechanism of Microwave Absorption}

When the microwave reaches the surface of absorber, it will experience three routes: absorption, reflection and transmission routes (Fig. 3a) [59]. The microwave will be reflected by the inner surfaces of the MAMs when microwave enters the inside of MAMs to optimize the internal microwave loss capability of MAMs to absorb microwave (Fig. 3b). However, the reflected and transmitted microwaves could bring secondary pollution. Therefore, one basic principle of designing MAMs is improving the absorption of incident wave to weaken the reflected and transmitted microwave.

Based on the electromagnetic energy conversion principle, there are two main ways to dissipate the microwave, namely dielectric loss and magnetic loss, respectively. The parameters used to represent dielectric loss and magnetic loss are permittivity $(\varepsilon)$ and permeability $(\mu)$, respectively. Both of them consist of real parts $\left(\varepsilon^{\prime}, \mu\right)$ and imaginary parts $\left(\varepsilon^{\prime \prime}, \mu^{\prime \prime}\right)$ : the real parts represent the ability of storing electrical or magnetic energy, while the imaginary parts represent the ability of energy dissipation (Eqs. 1 and 2) [69, 70].

$\varepsilon_{r}=\varepsilon^{\prime}-j \varepsilon^{\prime \prime}$

$\mu_{r}=\mu^{\prime}-j \mu^{\prime \prime}$

The dielectric loss tangent $\left(\tan \delta_{\mathrm{E}}\right)$ and magnetic loss tangent $\left(\tan \delta_{M}\right)$ are used to describe the dielectric loss capacity and magnetic loss capacity of the MAM, which can be expressed as Eqs. 3 and 4 [71, 72]:

$\tan \delta_{E}=\varepsilon^{\prime \prime} / \varepsilon^{\prime}$

$\tan \delta_{M}=\mu^{\prime \prime} / \mu^{\prime}$

The dielectric loss depends on the conductive loss and polarization loss (Fig. 3c). The former is caused by the induced current formed by the migration of free electrons inside the material under electromagnetic field. The MAMs will produce Joule effect when the induced current passed, so that the energy of electromagnetic wave is consumed in the form of heat energy. According to the free electron theory, the $\varepsilon$ " shows positive correlation with electrical conductivity ( $\sigma$ ) (Eq. 5) [73]. The latter is caused by the electromagnetic energy conversion during the polarization relaxation, which mainly includes the interfacial polarization caused by heterointerfaces and dipole polarization resulted from the surface charges, polar molecules and functional groups. According to Debye's law, the relationship between $\varepsilon^{\prime}$ and $\varepsilon^{\prime \prime}$ can be expressed by Eq. $6[74,75]$. The obtained $\varepsilon^{\prime}-\varepsilon^{\prime \prime}$ relationship curve is called Cole-Cole curve; the semicircles and upward tail in the curves represent the process of polarization relaxation and conductivity, respectively.

$\varepsilon^{\prime \prime}=\frac{\sigma}{2 \pi \varepsilon_{0} f}$

$\left(\varepsilon^{\prime}-\frac{\varepsilon_{s}+\varepsilon_{\infty}}{2}\right)^{2}+\left(\varepsilon^{\prime \prime}\right)^{2}=\left(\frac{\varepsilon_{s}-\varepsilon_{\infty}}{2}\right)^{2}$

where $\varepsilon_{s}$ is the static dielectric constant, $\varepsilon_{\infty}$ is the dielectric constant at infinite frequency, $\varepsilon_{0}$ is the dielectric constant in vacuum and $\sigma$ is the conductivity. 
Magnetic loss is the process of magnetization and reversal magnetization of magnetic materials in electromagnetic field, which converts electromagnetic energy into heat energy. In the frequency range of $2-18 \mathrm{GHz}$, the magnetic loss mainly includes hysteresis loss, eddy current loss, natural resonance and exchange resonance (Fig. 3d) [67, 73]. Hysteresis loss is caused by the displacement and rotation of the domain walls during magnetization, while it can be ignored in the weak magnetic field [67]. The eddy current loss can be evaluated by Eq. 7 [47, 66]. It can be proved the magnetic loss is mainly caused by eddy current loss if the value of $\mu^{\prime \prime}\left(\mu^{\prime}\right)^{-2} f^{-1}$ does not vary with $f$. Natural resonance is generated by the intrinsic anisotropic field of the MAM. The natural resonance linewidth is related to damping coefficient $[67,75,76]$. The natural resonance frequency is represented by Eq. 8, so that the natural resonance frequency can be adjusted by regulating the anisotropy constant of the MAM [67]. The effect of exchange resonance needs to be considered when the particles are submicron or nanometer scale, and the exchange formant frequency moves toward high frequency as the particle size decreases [75].

$f_{r}=\frac{2 \gamma K_{\text {eff }}}{M_{s}}$

$\mu^{\prime \prime}=\frac{2}{3} \pi \mu_{0}\left(\mu^{\prime}\right)^{2} \sigma d^{2} f$

where $\gamma, K_{e f f}$ and $M_{s}$ refer to gyromagnetic ratio, effective anisotropy constant and saturation magnetization, respectively. $\mu_{0}$ and $d$ are corresponding to the permeability of vacuum and thickness, respectively.

The MA property of MAM is determined by two factors: impedance matching and attenuation constant. The former determines the amount of microwaves that can enter the absorbers, while the latter is response for the dissipative ability to the incident microwave of the MAMs. Both of them are related to permittivity $(\varepsilon)$ and permeability $(\mu)$. When the input impedance $\left(Z_{i n}\right)$ of the MAM is equal to that of the free space $\left(Z_{0}\right)$, all of the microwaves arriving at the surface will enter into the MAM, and this phenomenon is called impedance matching. The calculation formulas are as follows:

$Z_{\text {in }}=Z_{0} \sqrt{\frac{\mu_{r}}{\varepsilon_{r}}} \tanh \left(\frac{2 \pi j f d}{c} \sqrt{\mu_{r} \varepsilon_{r}}\right)$
$|Z|=\left|\frac{Z_{\text {in }}}{Z_{0}}\right|$

In addition, the impedance matching can be characterized by a trigonometric function method as well, which is calculated by the following formulas $[42,71,77]$ :

$|\Delta|=\left|\sinh ^{2}(K f d)-M\right|$

$K=\frac{4 \pi \sqrt{\mu_{r} \varepsilon_{r}} \sin \frac{\delta_{E}+\delta_{M}}{2}}{c \cdot \cos \delta_{E} \cos \delta_{M}}$

$M=\frac{4 \mu^{\prime} \cos \delta_{E} \varepsilon^{\prime} \cos \delta_{M}}{\left(\mu^{\prime} \cos \delta_{E}-\varepsilon^{\prime} \cos \delta_{M}\right)^{2}+\left[\tan \left(\frac{\delta_{M}}{2}-\frac{\delta_{E}}{2}\right)\right]^{2}\left(\mu^{\prime} \cos \delta_{E}+\varepsilon^{\prime} \cos \delta_{M}\right)^{2}}$

The $\Delta$ function represents the impedance matching degree, and the ideal impedance matching will be achieved when the value of $\Delta$ is close to zero $(|\Delta| \leq 0.4)$.

The attenuation constant $(\alpha)$ is used to measure the microwave dissipation ability within the MAMs, which relies on the dielectric loss and magnetic loss capacity. Equation 14 is the calculation formula of attenuation constant [75-79]. It can be discovered that the attenuation constant was enlarged with the enhancement of dielectric loss and magnetic loss.

$\alpha=\sqrt{2} \frac{\pi f}{c} \times \sqrt{\left(\mu^{\prime \prime} \varepsilon^{\prime \prime}-\mu^{\prime} \varepsilon^{\prime}\right)+\sqrt{\left(\mu^{\prime \prime} \varepsilon^{\prime \prime}-\mu^{\prime} \varepsilon^{\prime}\right)^{2}+\left(\mu^{\prime} \varepsilon^{\prime \prime}+\mu^{\prime \prime} \varepsilon^{\prime}\right)^{2}}}$

To sum up, the index to evaluate the MA property is the reflection loss (RL) and the effective absorption bandwidth (EAB, RL $<-10 \mathrm{~dB}$ ); $90 \%, 99 \%$ and $99.9 \%$ of the electromagnetic wave are lost when the RL is less than $-10,-20$, and $-30 \mathrm{~dB}$, respectively. The RL can be calculated by Eq. $15[62,80,81]$ :

$R L=20\left|\log \frac{Z_{\text {in }}-1}{Z_{\text {in }}+1}\right|$

\section{MAMs Derived from MOFs}

According to the number of metallic elements in the center note, a variety of MOFs can be roughly divided in two categories: single-metal-based MOFs and multimetal-based MOFs. Moreover, the magnetic properties of metallic elements play a crucial role in the MA system. In this section, 


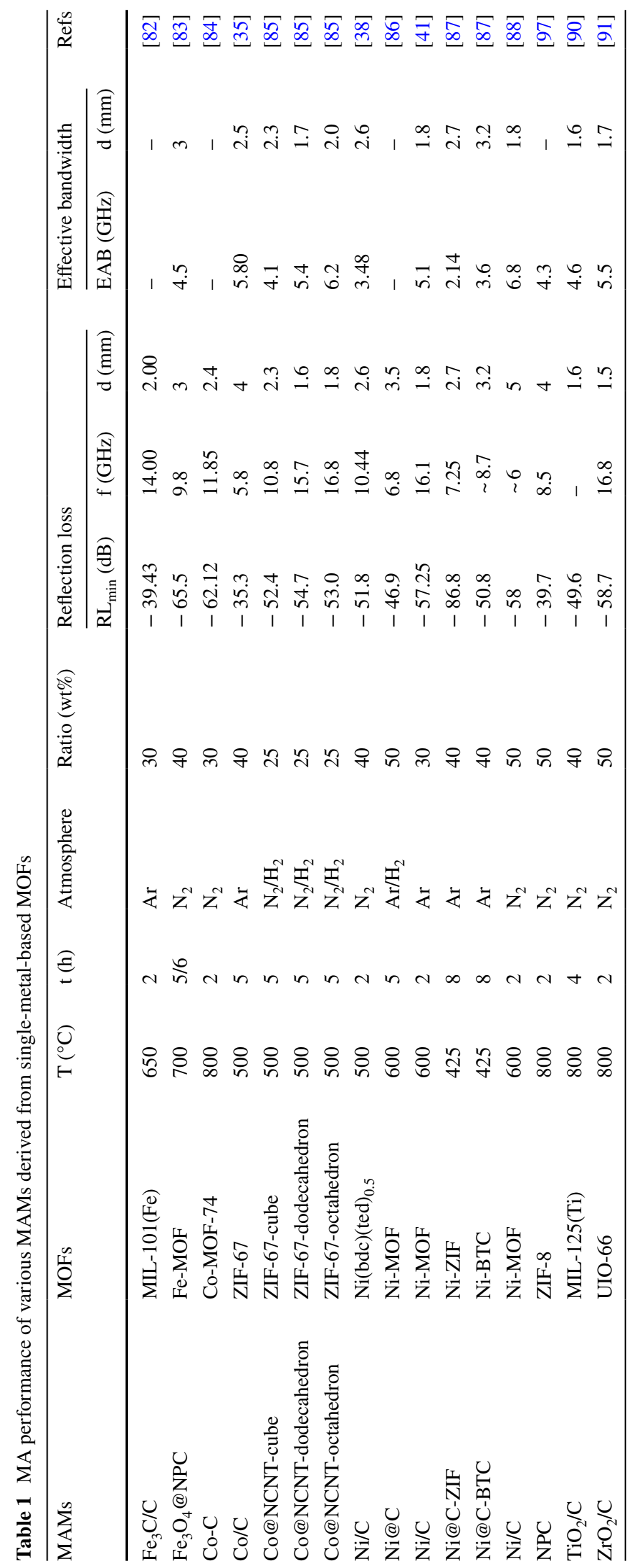



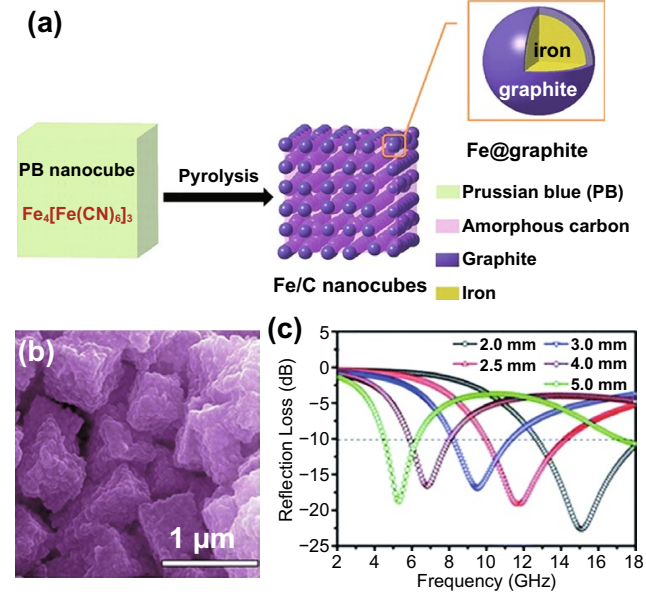

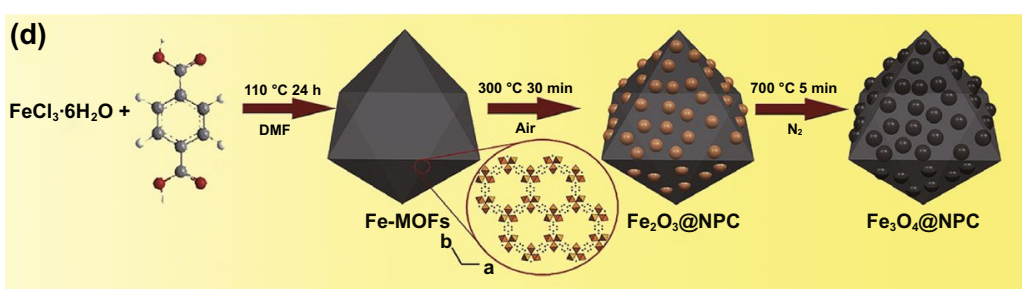

(e)

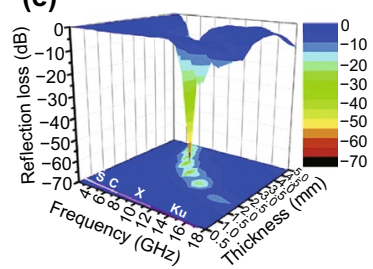

(f)

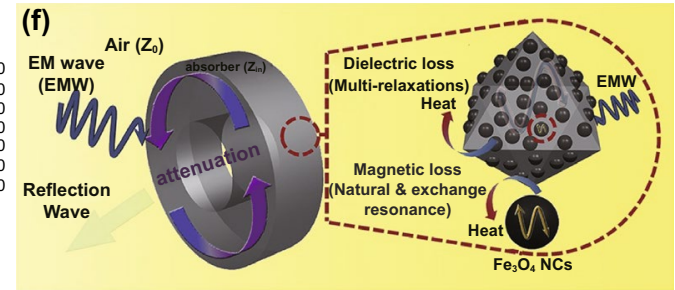

(g)

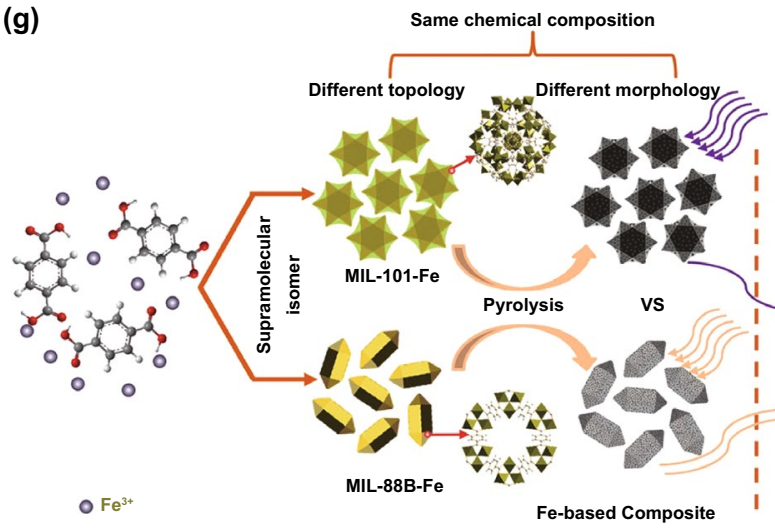

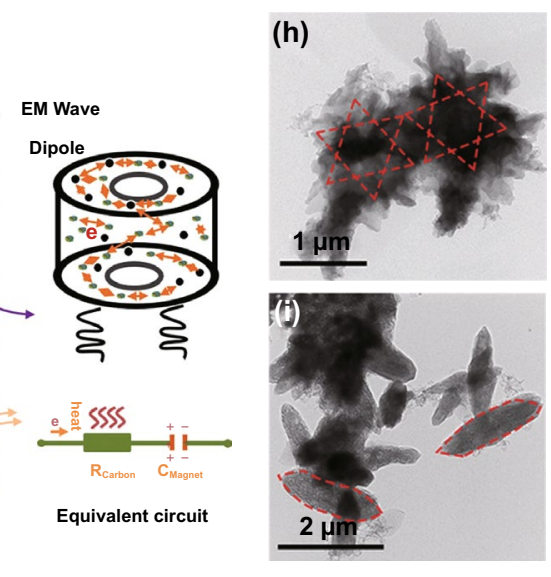

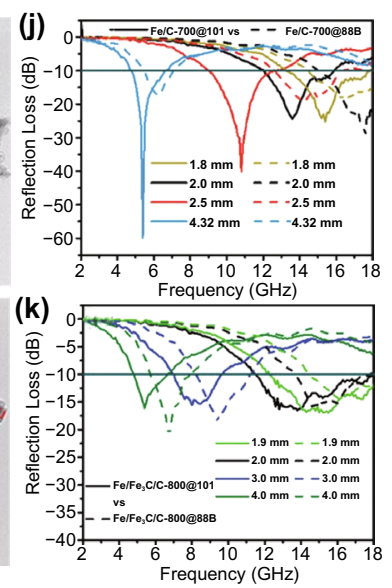

Fig. 4 a Synthesis method, b SEM image and c RL-f curves of Fe/C nanocubes. Reprinted with permission from Ref. [92] d Schematic diagram of the preparation and e RL-f curves of $\mathrm{Fe}_{3} \mathrm{O}_{4} @$ NPC. f Schematic illustration of the MA mechanism of $\mathrm{Fe}_{3} \mathrm{O}_{4} @$ NPC. Reprinted with permission from Ref. [83] g Schematic illustration for the synthetic process of the two kinds of FeC nanocomposites. TEM images and RL-f curves of $\mathbf{h}, \mathbf{j}$ FeC-800@101 and i, k FeC-800@88B. Reprinted with permission from Ref. [93]

the MAMs derived from single magnetic-metal-based, single nonmagnetic-metal-based MOFs and multimetal-based MOFs are introduced.

\subsection{MAMs Derived from Single-Metal-Based MOFs}

Usually, MAMs derived from magnetic-metal-based MOFs possess both electrical conductivity and magnetism, providing a convenient way for impedance matching and microwave attenuation. Iron ( $\mathrm{Fe})$, cobalt $(\mathrm{Co})$, nickel $(\mathrm{Ni})$ and their alloys all exhibit good magnetic properties, so that $\mathrm{Fe}-$, $\mathrm{Co}-$, and Ni-based MOFs are studied extensively in the microwave absorption field. Different kinds of MAMs derived from single-metal-based MOFs are listed in Table 1.

\subsubsection{Iron-Based MOFs as Precursors}

Because of the low cost and excellent magnetic property of iron, Fe-based MOFs are considered as good precursors of MAMs. In 2015, Qiang and co-workers used $\mathrm{Fe}_{4}\left[\mathrm{Fe}(\mathrm{CN})_{6}\right]_{3}$ (PB) as precursors of $\mathrm{Fe} / \mathrm{C}$ nanocubes $(\mathrm{Fe} / \mathrm{C})$, which constituted by the circular nanoparticles with iron as core and graphite as shell (Fig. 4a-b) [92]. The as-prepared Fe/C possessed an $\mathrm{RL}_{\text {min }}$ of $-22.6 \mathrm{~dB}$ at $15.0 \mathrm{GHz}$ and $\mathrm{EAB}$ of $5.3 \mathrm{GHz}$ (Fig. 4c). Xiang at el. fabricated nanoporous $\mathrm{Fe}_{3} \mathrm{O}_{4} @ \mathrm{C}\left(\mathrm{Fe}_{3} \mathrm{O}_{4} @ \mathrm{NPC}\right)$ composites using a novel two-step thermal decomposition method [83]. As shown in Fig. 4d, the $\mathrm{Fe}_{2} \mathrm{O}_{3} @ \mathrm{NPC}$ was obtained after thermal treatment of $\mathrm{Fe}-\mathrm{MOF}$ at $300{ }^{\circ} \mathrm{C}$ in air, the $\mathrm{Fe}_{2} \mathrm{O}_{3}$ was transferred to $\mathrm{Fe}_{3} \mathrm{O}_{4}$ after the subsequent pyrolysis in $\mathrm{N}_{2}$, and the organic residues 

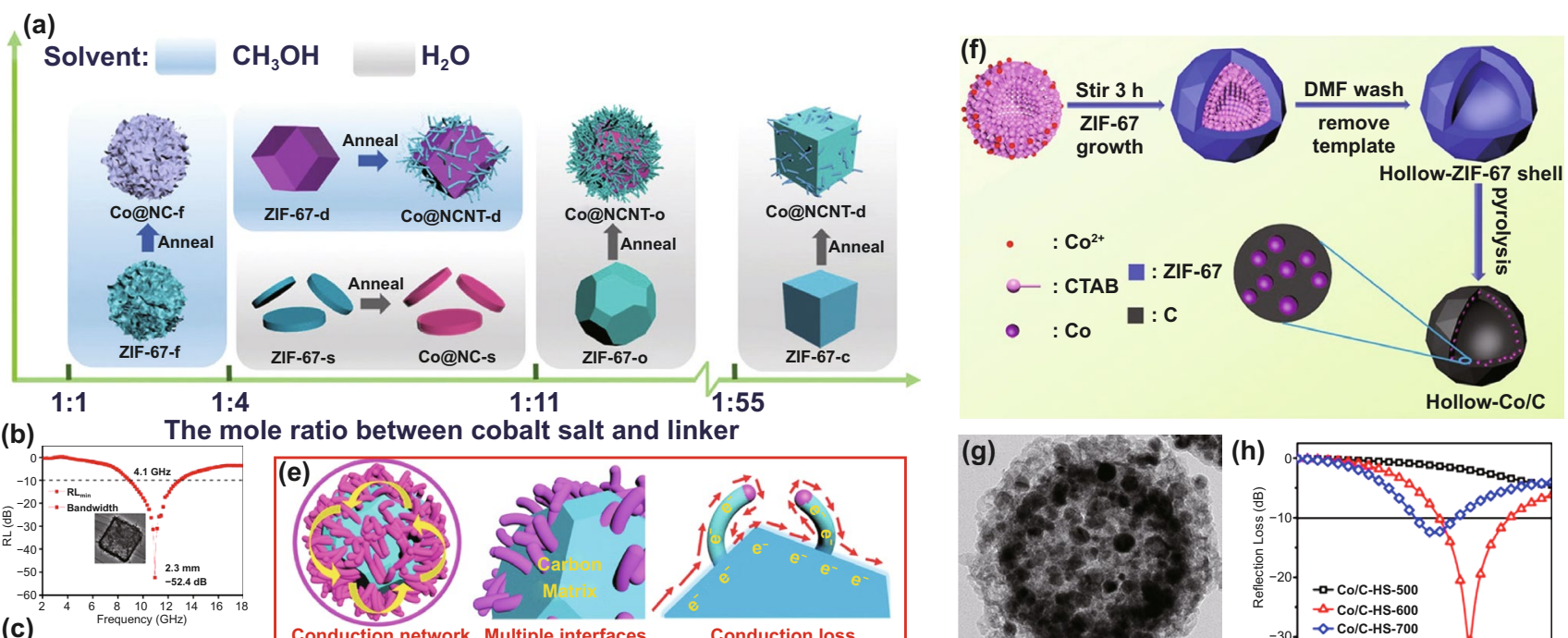

he mole ratio between cobalt salt and linker
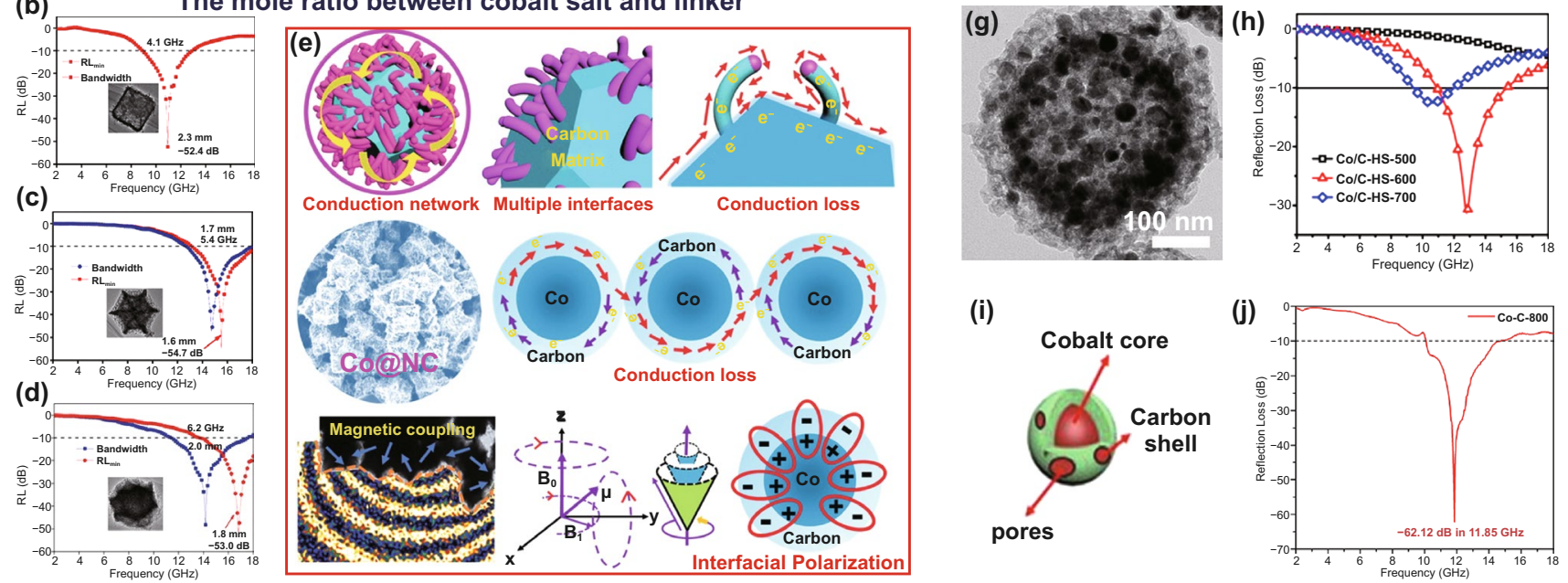

Fig. 5 a Schematic preparation process of Co@NCNT composites. RL-f curves of the b Co@NCNT-cube, c Co@NCNT-dodecahedron and d Co@NCNT-octadecahedron. e Microwave loss mechanism diagram of Co@NCNTs. Reprinted with permission from Ref. [85]. f The synthesis route, $\mathbf{g}$ TEM image and $\mathbf{h}$ RL-f curves of hollow Co/C microspheres. Reprinted with permission from Ref. [42]. i Structure diagram and $\mathbf{j}$ RL-f curve of C/Co composite. Reprinted with permission from Ref. [84]

in $\mathrm{Fe}_{2} \mathrm{O}_{3} @ \mathrm{NPC}$ could act as reductant to promote the reduction of $\mathrm{Fe}^{3+}$ to $\mathrm{Fe}^{2+}$. The $\mathrm{Fe}_{3} \mathrm{O}_{4} @ \mathrm{NPC}$ achieved excellent MA performance with $\mathrm{RL}_{\min }$ of $-65.5 \mathrm{~dB}$ at $9.8 \mathrm{GHz}$ as well as $\mathrm{EAB}$ of $4.5 \mathrm{GHz}$ (Fig. 4e). The synergy of magnetic and dielectric mechanisms insured favorable impendence matching, and the dual energy attenuation mechanisms provided the conditions of electromagnetic energy transformation (Fig. 4f). Miao et al. prepared a series of MAMs using octahedral MIL-101-Fe and rod-like MIL-88B-Fe as precursors [93]. As shown in Fig. 4g-i, the two precursors were heated under inert atmosphere to obtain FC-T@101 and FC-T@ 88B ( $T$ represented the temperature during the pyrolysis process, $T=600,700$, and $800^{\circ} \mathrm{C}$ ), respectively. The experimental result indicated that FC-600@101 and FC-600@88B did not exhibit effect of RL because of their low permittivity. The higher pyrolysis temperature could obviously enhance the permittivity of Fe-based MOF-derived MAMs. Compared with FC-700@88B and FC-800@88B, the two counterparts derived from MIL-101-Fe presented better MA performance, because the abundant graphite layers could be in situ generated during the pyrolysis of MIL-101-Fe and enhance the conductive loss effectively. Particularly, the $\mathrm{RL}_{\min }$ and EAB of FC-700@101 reached up to $-59.2 \mathrm{~dB}$ and $6.5 \mathrm{GHz}$, respectively (Fig. 4j-k). During pyrolysis of Fe-based MOFs in an inert atmosphere, the $\mathrm{Fe}^{2+}$ can be converted to $\mathrm{Fe}_{3} \mathrm{O}_{4}$, which can further be transformed to $\mathrm{Fe}_{3} \mathrm{C}[94,95]$. However, the presence of $\mathrm{Fe}_{3} \mathrm{C}$ could decrease the magnetism, so that reasonable preparation conditions should be designed to insure the low content of $\mathrm{Fe}_{3} \mathrm{C}$ [92].

\subsubsection{Cobalt-Based MOFs as Precursors}

It is well known that the whole MA performance of MAM is highly dependent on the morphology of MOF precursor. As a typical Co-based MOF, ZIF-67 presents high potential in MA field because of its ultrahigh porous volume. 
Moreover, the morphology of ZIF-67 can be precisely controlled by tuning key parameters such as solvent and metal content in the synthesis process; thus, ZIF-67-derived MAMs with varying morphologies have been widely investigated. In 2020, Huang and co-workers prepared various ZIF-67 with different morphologies including cube, dodecahedron, octadecahedron, flower and sheet, and then, the corresponding Co@NCNTs were obtained after pyrolysis (Fig. 5a) [85]. As indicated in Fig. 5b-d, the $\mathrm{RL}_{\text {min }}$ of Co@NCNT-cube, Co@NCNT-dodecahedron and Co@ NCNT-octadecahedron were $-52.4,-54.7$ and $-53.0 \mathrm{~dB}$, respectively, which were better than those of Co@NCNTsflower and Co@NCNTs-sheet, because a plenty of planes provided large surface area of the former three, promoting microwaves enter inside. The MA attenuation mechanism is shown in Fig. 5e, the CNTs on the surface formed connected conductive network and provided conductive loss together with the graphitized carbon matrix; Co nanoparticles exhibited significant magnetic coupling effects and the defects and $\mathrm{N}$-doped sites could be used as polarization centers (Fig. 5e). Besides, the hollow-ZIF-67 was synthesized using a one-step template method, which can be calcinated to receive hollow $\mathrm{Co} / \mathrm{C}$ microspheres (Fig. $5 \mathrm{f}-\mathrm{g}$ ) [42]. Compared with the normal dodecahedron-like $\mathrm{Co} / \mathrm{C}$ composites, the hollow $\mathrm{Co} / \mathrm{C}$ microspheres exhibited better MA performance; the $\mathrm{RL}_{\min }$ was $-31.3 \mathrm{~dB}$ at $12.8 \mathrm{GHz}$ when calcined at $600{ }^{\circ} \mathrm{C}$ (Fig. 5h). There were mainly two reasons for this experimental phenomenon. Firstly, the higher porous volume was beneficial to the construction of interconnected conductive network and interface, improving the multiple reflection of microwave. Besides, the internal cavity suppressed the interaction among carbon matrix and the incident microwave, restricting the generation of the induced magnetic field and improving the magnetic loss.

Apart from ZIF-67, other Co-based MOFs have also been applied as precursor of MAMs. In 2018, Wang and co-workers synthesized $\mathrm{Co}-\mathrm{C}$ core-shell nanocomposite through carbonization of Co-MOF-74; the prepared Co-C exhibited exceptional MA property with a $\mathrm{RL}_{\min }$ of $-62.12 \mathrm{~dB}$ (a)

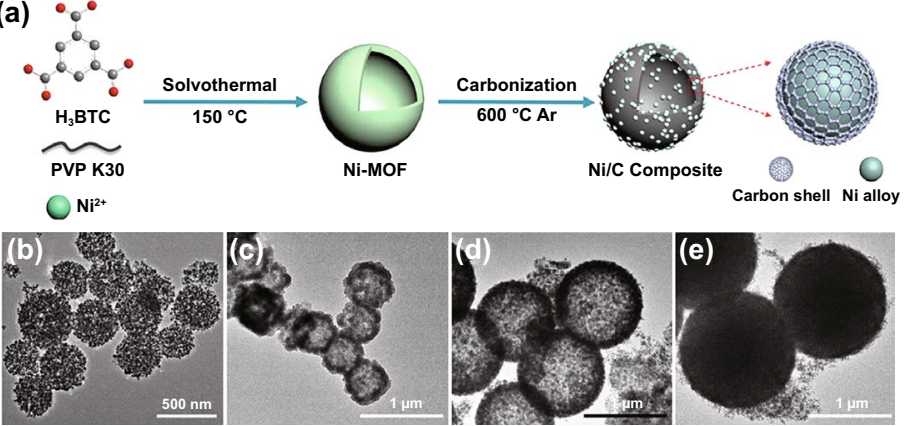

(g)
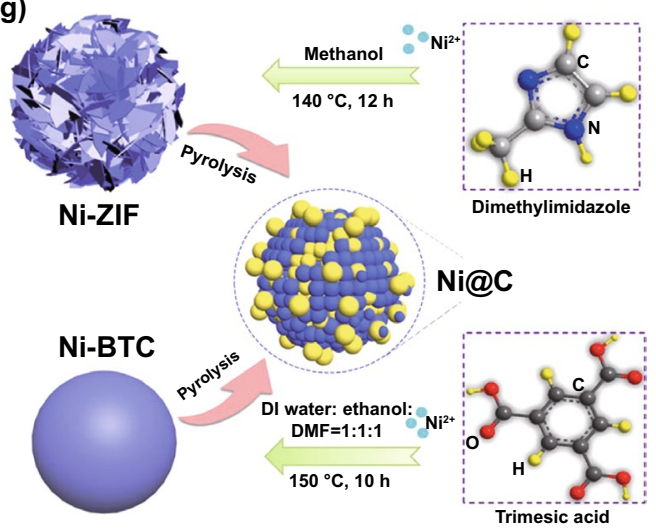

(f)

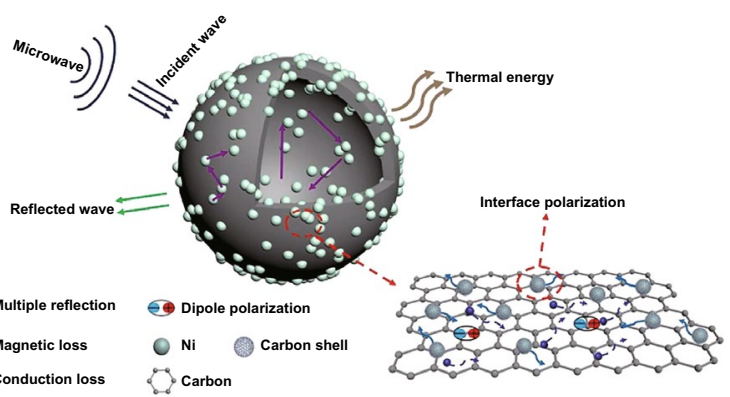

a. $\rightarrow$ Conduction loss $\quad$ C Carbon

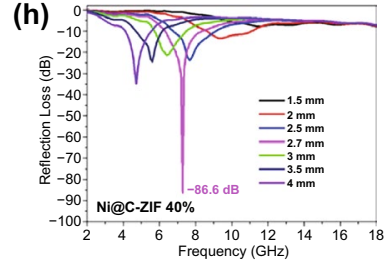

(i)

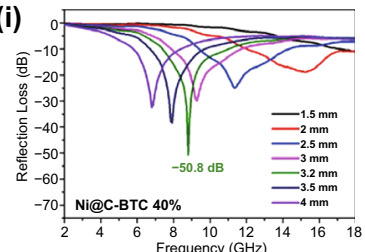

(j)

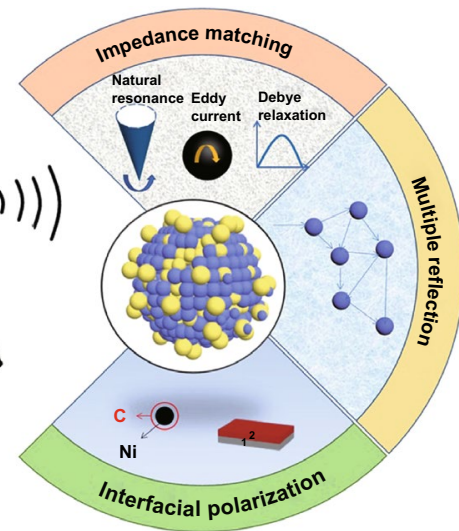

Fig. 6 a Diagrammatic sketch of the preparation process of Ni/C composites. b-e TEM images of four kinds of Ni/C composites. $\mathbf{f}$ Illustration of MA mechanism for Ni/C composites. Reprinted with permission from Ref. [41]. g Schematic illustration of the fabrication process of Ni@C microspheres. RL-f curves of $\mathbf{h}$ Ni@C-ZIF and i Ni@C-BTC. j Schematic diagram of MA mechanism for Ni@C microspheres. Reprinted with permission from Ref. [87] 
at the frequency of $11.85 \mathrm{GHz}$ (Fig. 5i-j)[84]. Besides, a flake-assembled jujube pit-shaped C/Co composite by annealing a Co-based MOF displayed an outstanding $\mathrm{RL}_{\text {min }}$ with $-40.0 \mathrm{~dB}$ at $2.8 \mathrm{~mm}$ [96].

\subsubsection{Nickel-Based MOFs as Precursors}

Apart from Fe- and Co-based MOFs, Ni-based MOFs are also important precursors of MAMs. As depicted in Fig. 6a, the $\mathrm{Ni} / \mathrm{C}$ spheres were directly prepared by heating Ni-MOFs at $600{ }^{\circ} \mathrm{C}$ under Ar atmosphere [41]. The mixture of DMF and water was used as an solvent, and the diameter of prepared $\mathrm{Ni} / \mathrm{C}$ spheres was gradually enlarged with the increase in water content in mixture because the nucleation rate of $\mathrm{H}_{3} \mathrm{BTC}$ in DMF was faster than that in water (Fig. 6b-e). Among these $\mathrm{Ni} / \mathrm{C}$ spheres, the optimal $\mathrm{RL}_{\text {min }}$ could reach to $-57.25 \mathrm{~dB}$ at $16.1 \mathrm{GHz}$, while the thickness was only $1.8 \mathrm{~mm}$. The great MA property of $\mathrm{Ni} / \mathrm{C}$ spheres originated from the synergistic effect of multiple reflection, magnetic loss, conductive loss and dipole polarization (Fig. 6f). In 2019, the effect of ligands on the MA property of MOF-derived MAMs was also investigated by Yan and $c o$-workers [87]. As shown in Fig. 6g, the dimethylimidazole and trimesic acid were utilized as ligands to prepare Ni-ZIF with spherical-like hierarchical 3D nanostructures and Ni-BTC with smooth and complete microsphere morphology, respectively. The experimental results revealed that $\mathrm{Ni} @ \mathrm{C}$ ZIF and Ni@C-BTC both exhibited superior MA properties in the frequency of 2 to $18 \mathrm{GHz}$ (Fig. $6 \mathrm{~h}-\mathrm{i}$ ). In particular, the $\mathrm{RL}_{\text {min }}$ of Ni@C-ZIF reached to $-86.8 \mathrm{~dB}$ at $13.2 \mathrm{GHz}$, which was mainly attributed to $\mathrm{N}$-contained ligands resulting in the improvement of interfacial polarization. As shown in Fig. 6j, the appropriate magnetic loss and dielectric loss endowed the MAM with excellent impedance matching, and the multiple reflection and interfacial polarization also played significant roles in the microwave dissipation. Moreover, Zeng et al. used dimethylimidazole and pyromellitic acid as the mixed ligands to prepare Ni-based MOF as precursor of $\mathrm{Ni} @ \mathrm{C}$ nanocomposite in 2021 [86]. The $\mathrm{RL}_{\text {min }}$ of $\mathrm{Ni} @ \mathrm{C}$ was obtained when the pyrolysis temperature was $600{ }^{\circ} \mathrm{C}(-46.9 \mathrm{~dB}$ at $3.5 \mathrm{~mm})$.

\subsubsection{Single Nonmagnetic-Metal-Based MOF-Derived MAMs}

Recently, some nonmagnetic-metal-based MOFs have been attempted as precursors of MAMs. Due to the absence of magnetic loss medium, the selection of precursors and the design of the experimental process are both based on the adjustment of dielectric constant and microstructure. At present, the main research mainly includes the following.

Due to the relative low melting point $\left(420^{\circ} \mathrm{C}\right)$ and boiling point $\left(908{ }^{\circ} \mathrm{C}\right), \mathrm{Zn}$-based MOFs are increasingly concentrated. The $\mathrm{Zn}$ element in $\mathrm{Zn}$-based MOFs could convert to $\mathrm{ZnO}$ when the calcination temperature is higher than $550{ }^{\circ} \mathrm{C}$ [97]. $\mathrm{ZnO}$, a typical polarization semiconductor with low electrical conductivity and wide bandgap, has been extensively used in adjusting MA property [98-100]. Besides, the carbon-reduced $\mathrm{Zn}$ metal would start to be vaporized when the pyrolysis temperature exceeded $800{ }^{\circ} \mathrm{C}$, leading to the formation of amorphous structure to increase the porosity of the MAMs [89]. In 2018, Wu calcined ZIF-8 at three different temperatures $\left(700,800\right.$ and $\left.900^{\circ} \mathrm{C}\right)$ and the products named NPC700, NPC800 and NPC900, respectively [97], in which NPC800 owned the largest $\mathrm{RL}_{\min }(-39.7 \mathrm{~dB})$ because of its appropriate porosity and well-maintained skeleton. However, the MW properties of Zn-based MOFderived MAMs are greatly restricted to the absence of magnetism. So far, rarely studies have been focused on MAMs derived from pure $\mathrm{Zn}$-contained MOF; however, introducing magnetic components in $\mathrm{Zn}$-contained MOF and combining $\mathrm{Zn}$ and magnetic metals as mixed centers to form multimetal MOF as MAMs precursor have attracted tremendous research attention in microwave absorption field.

As typical ceramic dielectric materials, $\mathrm{ZrO}_{2}$ and $\mathrm{TiO}_{2}$ show great application potentials in MA field due to their excellent chemical stability, environmental friendliness and mass storage [101]. Moreover, the $\mathrm{ZrO}_{2}$ and $\mathrm{TiO}_{2}$ in the nanocomposites played a key role in achieving good impedance matching because their relatively low electrical conductivity could neutralize the excessive conductive loss of the graphitized carbon [101]. Therefore, the $\mathrm{Zr}$ - and Tibased MOFs grasped an increasing attention in MA field because the excellent thermal stability enables them to form metal oxides rather than metallic state during carbonization [101]. For instance, Zhang and co-workers obtained $\mathrm{ZrO}_{2} / \mathrm{C}$ composites after the carbonization of UIO-66 with excellent MA performance; the optimized $\mathrm{RL}_{\text {min }}$ and $\mathrm{EAB}$ were $-58.7 \mathrm{~dB}(16.8 \mathrm{GHz}, 1.5 \mathrm{~mm})$ and $5.5 \mathrm{GHz}$ (Fig. 7ac), respectively [91]. Ma and co-workers used MIL-125(Ti) as the precursor to obtain $\mathrm{TiO}_{2} / \mathrm{C}$ nanocomposites with an $\mathrm{RL}_{\text {min }}$ of $-49.6 \mathrm{~dB}$ and $\mathrm{EAB}$ of $4.6 \mathrm{GHz}$, respectively [90]. Figure $7 \mathrm{~d}-\mathrm{e}$ shows the TEM images and RL-f curves of 
(a)
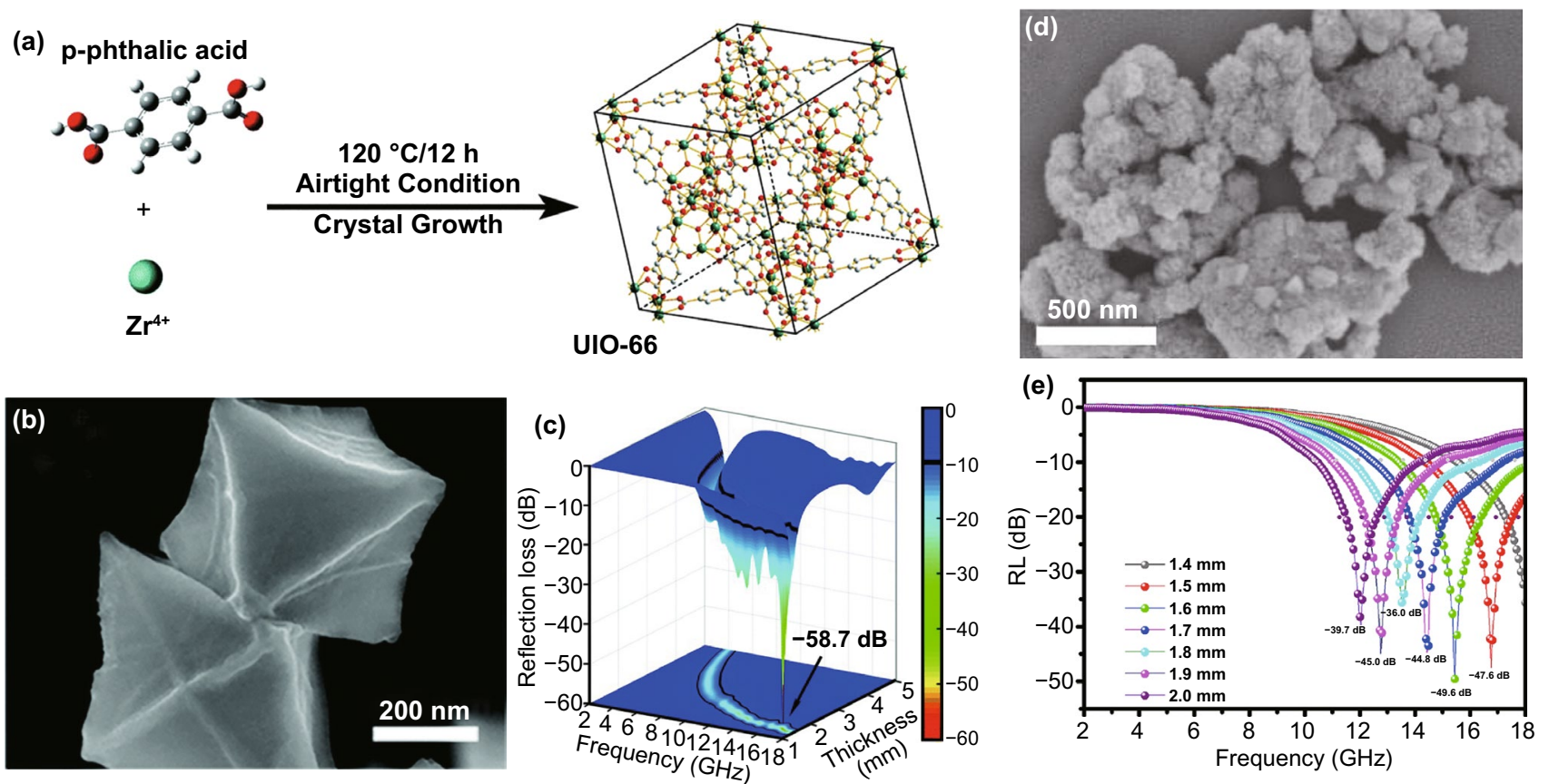

(f)
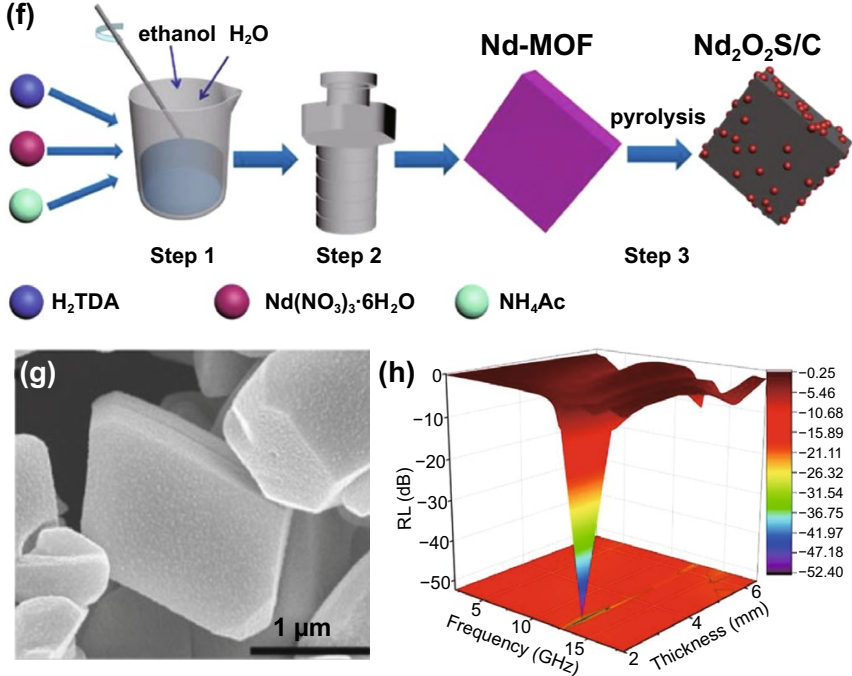

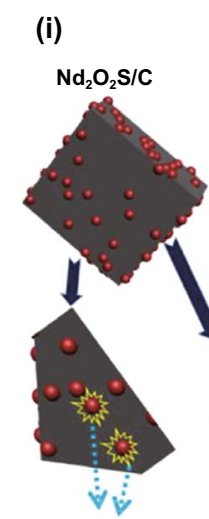

Interfacial polarization

? Defect e Free electron

- $\mathrm{Nd}_{2} \mathrm{O}_{2} \mathrm{~S}$

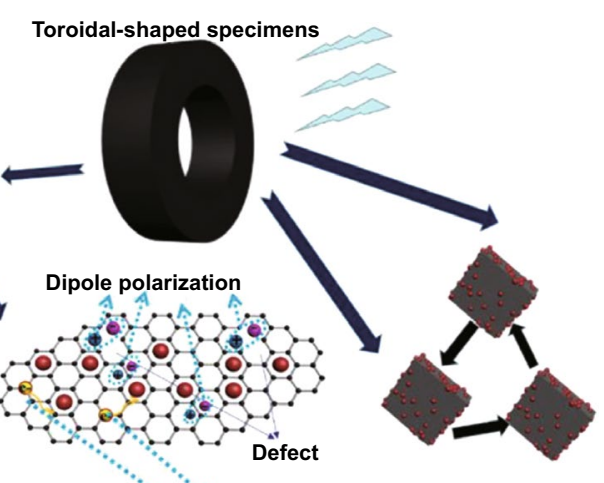

Multiple reflection

Dipole $\cdot C$

Fig. 7 a Schematic illustration of diagram and crystal structure of UIO-66. b TEM image and c 3D RL-f curves of $\mathrm{ZrO}_{2} / \mathrm{C}$. Reprinted with permission from Ref. [91]. d TEM image and e RL-f curves of $\mathrm{TiO}_{2} / \mathrm{C}$. Reprinted with permission from Ref. [90]. f Diagrammatic sketch of the preparation process, $\mathbf{g}$ SEM image, $\mathbf{h}$ 3D RL-f curves and $\mathbf{i}$ the MA mechanism of $\mathrm{Nd}_{2} \mathrm{O}_{2} \mathrm{~S} / \mathrm{C}$. Reprinted with permission from Ref. [103]

$\mathrm{TiO}_{2} / \mathrm{C}$ nanocomposites. Except for the enhanced impedance matching, the natural porous structure of MIL-125 also made nonnegligible contribution to the internal reflection of $\mathrm{TiO}_{2} / \mathrm{C}$. The rare-earth oxides such as $\mathrm{CeO}_{2}$ and $\mathrm{Nd}_{2} \mathrm{O}_{3}$ also exhibited improved MA properties through combining with carbon materials [102]. Shen and $c o$-workers synthesized an ingenious rhombic $\mathrm{Nd}_{2} \mathrm{O}_{2} \mathrm{~S} / \mathrm{C}$ nanocomposite by direct thermal treatment of Nd-MOF (Fig. 7f-g) [103]. The
$\mathrm{RL}_{\min }$ of $\mathrm{Nd}_{2} \mathrm{O}_{2} \mathrm{~S} / \mathrm{C}$ carbonized at $800{ }^{\circ} \mathrm{C}$ reached $-52.3 \mathrm{~dB}$ at $2.56 \mathrm{~mm}$ thickness (Fig. $7 \mathrm{~h}$ ). In addition to the conductive loss originated from the graphitized carbon matrix, the interfacial polarization caused by many heterogeneous interfaces between $\mathrm{Nd}_{2} \mathrm{O}_{2} \mathrm{~S}$ and porous carbon and the dipole polarization originated from carbon defects and oxygen-containing functional groups also played crucial roles in enhancing MA capacity (Fig. 7i). 
(a)
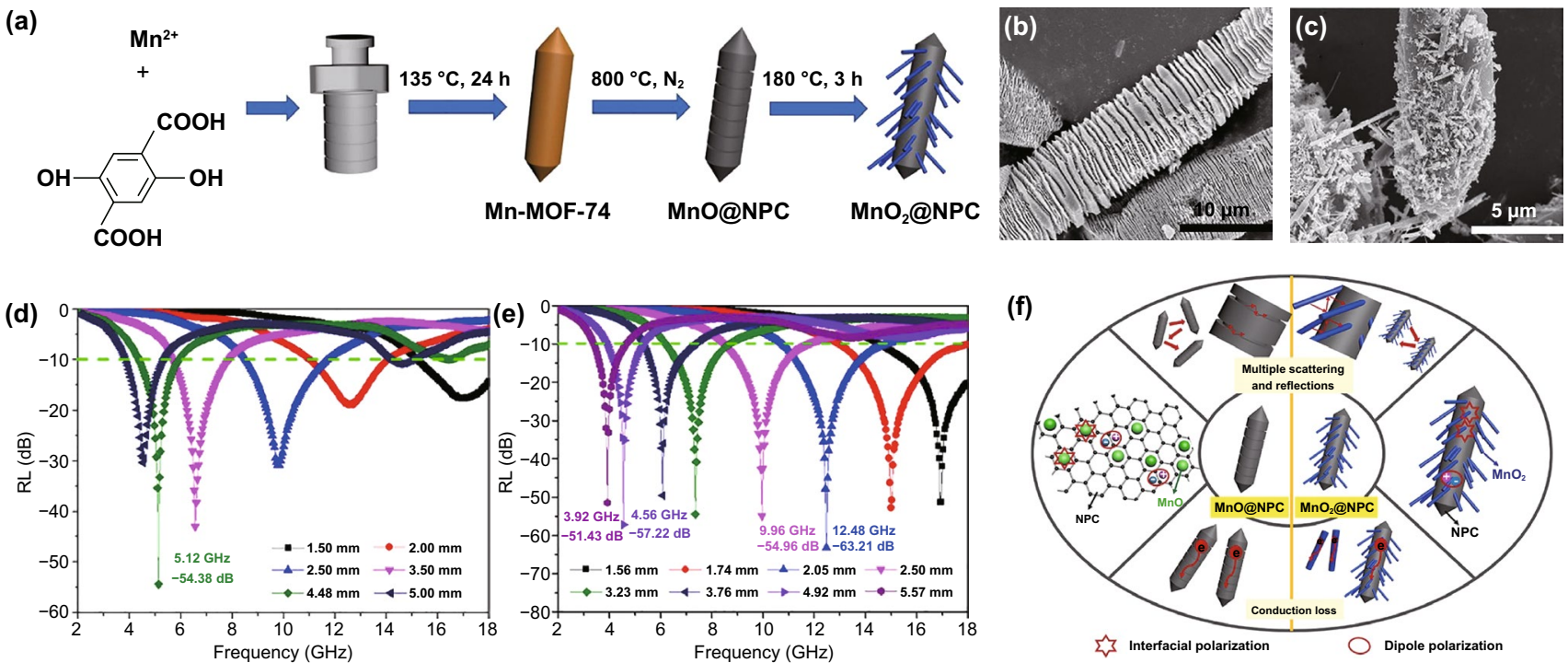

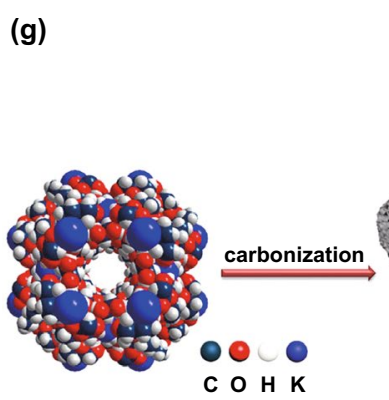

Y-CD-MOF-K

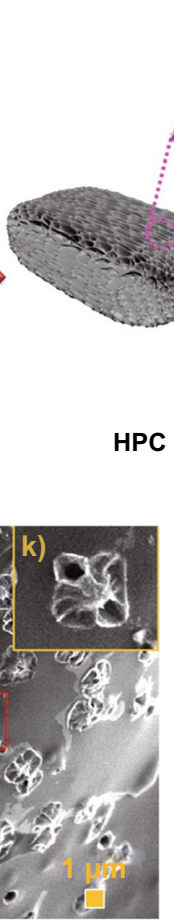

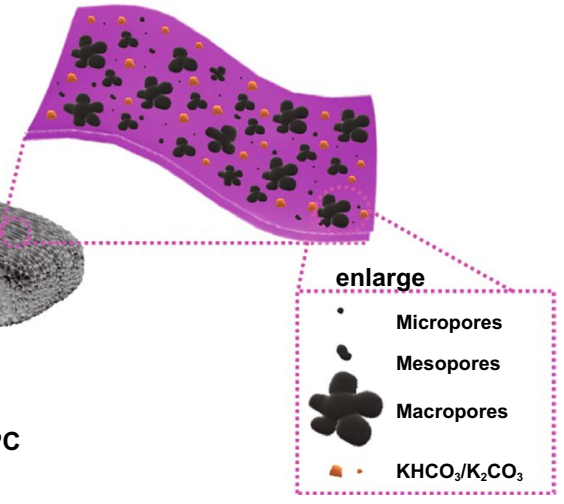

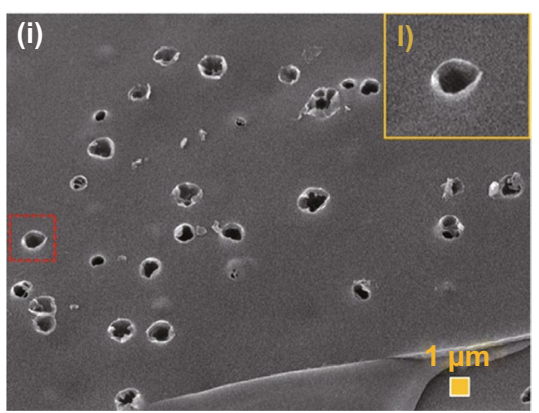

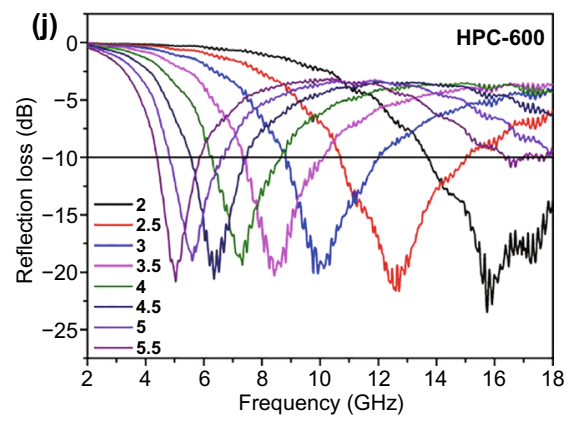

(k)
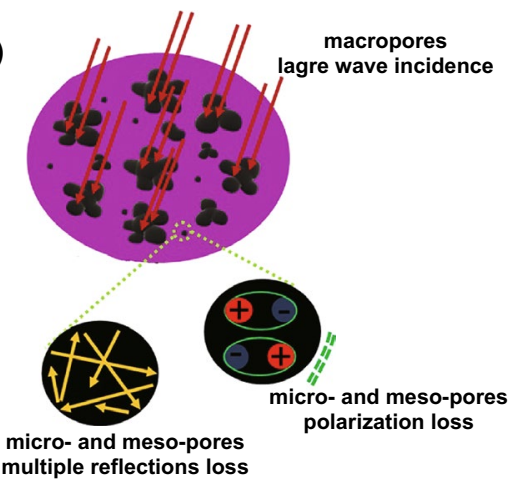

Fig. 8 a Schematic illustration of diagram of MnO@NPC and $\mathrm{MnO}_{2} @ \mathrm{NPC}$. TEM image and 3D RL-f curves of b, d MnO@NPC and c, e $\mathrm{MnO}_{2} @ \mathrm{NPC}$. f Schematic illustration of MA mechanisms for MnO@NPC and $\mathrm{MnO}_{2} @ \mathrm{NPC}$. Reprinted with permission from Ref. [108]. g Preparation process diagram of $\boldsymbol{\gamma}$-CD-MOF-K-derived HPC. TEM images of $\mathbf{h}$ HPC-600 and $\mathbf{i}$ HPC-900. $\mathbf{j}$ RL-f curves and $\mathbf{k}$ MA mechanism of $\mathrm{TiO}_{2} /$ C. Reprinted with permission from Ref. [109]

Nowadays, manganese oxides have been increasingly studied in the fields of MA owing to their environmental friendliness, abundant resources and unique physical and chemical properties [104]. $\mathrm{MnO}$, a semiconductor with wide bandgap of $3.6 \mathrm{eV}$, has low conductivity and weak attenuation ability, exhibiting excellent wave-transparence
$[105,106] . \mathrm{MnO}_{2}$ is also a peculiar metallic semiconductor with great metal-like electrical conductivity and dielectric property [107]. Liu's group synthesized MnO@NPC and $\mathrm{MnO}_{2} @ \mathrm{NPC}$ with Mn-MOF-74 as precursors [108]. The caterpillars-like MnO@NPC was prepared by directly carbonizing Mn-MOF-74 at $800{ }^{\circ} \mathrm{C}$, and the hedgehog-like 
$\mathrm{MnO}_{2} @ \mathrm{NPC}$ was obtained after the subsequent hydrothermal reaction of MnO@NPC (Fig. 8a-c). Both MnO@ NPC and $\mathrm{MnO}_{2} @ \mathrm{NPC}$ achieved surprising MA performance, especially all the $\mathrm{RL}_{\min }$ of $\mathrm{MnO}_{2} @ \mathrm{NPC}$ was higher than $-50 \mathrm{~dB}$ in S, C, X, and Ku bands (Fig. 8d-e). In this system, the significant anisotropy and high aspect ratio of $\mathrm{MnO}_{2} @$ NPC were conducive to the formation of efficient conductive network, and the dipole polarization was also enhanced by the additional dipole centers resulted from oxidation of MnO@NPC, which skillfully optimized the MA performance of $\mathrm{MnO}_{2} @ \mathrm{NPC}$ (Fig. 8f).

Some other metal-centered MOFs have also been attempted as precursors for MAMs due to their unique properties. For instance, Zhang et al. fabricated ingenious hierarchical porous carbon (HPC) materials using $\gamma$-CDMOF-K as carbonizing template with $\mathrm{K}^{2+}$ acted as activator in the formation of HPC (Fig. 8g) [109]. The HPC600 was obtained by directly pyrolyzing $\gamma$-CD-MOF-k at $600{ }^{\circ} \mathrm{C}$, and the HPC-600-900 was fabricated by calcining HPC-600 at $900{ }^{\circ} \mathrm{C}$ subsequently. As shown in Fig. $8 \mathrm{~h}$ and $\mathrm{i}$, the flower-like pore structure and ordinary circular pore structure were observed on the surface of HPC-600 and HPC-600-900, respectively. The HPC-600 showed effective MA property with a $\mathrm{RL}_{\text {min }}$ of $-23.5 \mathrm{~dB}$ and $\mathrm{EAB}$ of $4.3 \mathrm{GHz}$, while the MA property of HPC-600-900 was poor (Fig. 8j). Pore structure and pore size distribution had great influence on their impendence matching and electromagnetic energy attenuation, in which the flower-like pore could bring in more heterogeneous interfaces and the abundant microand mesopores were in favor of the multiple reflection loss and polarization loss (Fig. 8k). Besides, some familiar metal ions have also been tried as the centers of MOF precursors. For instance, Ji's group reported a $\mathrm{MgO} / \mathrm{C}$ composite derived from CPO-27-Mg [110]. The $\mathrm{RL}_{\text {min }}$ of $\mathrm{MgO} / \mathrm{C}$ was $-14.93 \mathrm{~dB}$ at the $2.0 \mathrm{~mm}$ and the EAB achieved $4.9 \mathrm{GHz}$.

\subsection{MAMs Derived from Multimetal-Based MOFs}

For single magnetic-metal-based MOFs, the type and amount of metal ions are relatively fixed, so the permeability and permittivity of MAMs derived from them can only be adjusted by changing the heating temperature. Therefore, using multimetal-based MOFs as precursors can effectively avoid this problem, because the electromagnetic parameters of MAMs could be effectively adjusted by changing the ratio of different metals. Besides, the morphologies of multimetal-based MOFs can be easily tuned when metal ions with different valence states share the mental center/cluster, because the different coordination numbers and geometries can influence the growth rate of building units $[111,112]$. The multimetal-based MOF precursors can be divided into these basic three types: (i) multi-magnetic-metal-based MOFs, (ii) multi-nonmagnetic-metal-based MOFs and (iii) mixed-metal-based MOFs. The MA properties of the MAMs derived from above multimetal-based MOFs are summarized in Table 2.

\subsubsection{Multi-magnetic Metal MOFs as Precursors}

One confront challenge is that the impedance mismatch of MOF-derived MAMs was caused by the sufficient permittivity and inadequate permeability. Using two or more magnetic metal ions as mental center/cluster is a promising method to moderate this issue because the various magnetic mental and alloy nanoparticles can be generated during pyrolysis process. In 2020, Wang and co-workers prepared three different $\mathrm{CoFe}-\mathrm{MOF}-74$ nanocomposites by simply adjusting the molar ratio of $\mathrm{Co} / \mathrm{Fe}$ [122]. As shown in Fig. 9a, the samples shown rod-like, nest-like and sheet-like morphologies when the $\mathrm{Co} / \mathrm{Fe}$ was 4:0, 3:1 and 2:2, respectively. The CoFe@C composites derived from the above samples all exhibited great attenuation abilities of MW, which originated from the magnetic loss of $\mathrm{Co} / \mathrm{Fe}$ alloy, conductive loss of graphitized carbon, multiple reflection supplied by porous structure and polarization loss of heterogeneous interfaces and defects (Fig. 9b). The MA properties of these three $\mathrm{CoFe} @ \mathrm{C}$ nanomaterials were $-55.5 \mathrm{~dB}$ at $10.6 \mathrm{GHz},-61.8 \mathrm{~dB}$ at $12.7 \mathrm{GHz},-47.5 \mathrm{~dB}$ at $13.9 \mathrm{GHz}$, and the EAB was 5.4, 9.2 and 7.4 GHz, respectively (Fig. 9ce). The relatively better performance of hierarchical nest-like $\mathrm{CoFe} @ \mathrm{C}$ benefited from its porous structure and reasonably constitute. Similarly, Ji’s group synthesized a CoNi@C sample using CoNi-MOF as precursor, obtaining a $\mathrm{RL}_{\text {min }}$ of $-43.7 \mathrm{~dB}$ with a low thickness of $1.7 \mathrm{~mm}$ [123]. In 2019, the hollow FeCoNi@C nanocomposite was synthesized through roasting the flowered spherical MOF containing $\mathrm{Fe}, \mathrm{Co}$, and Ni multimetal ions at high temperature (Fig. 9f) [116]. The molar ratio of each component in the FeCoNi alloy was varied with the carbonization temperature, significantly affecting the lattice parameters and the magnetic 


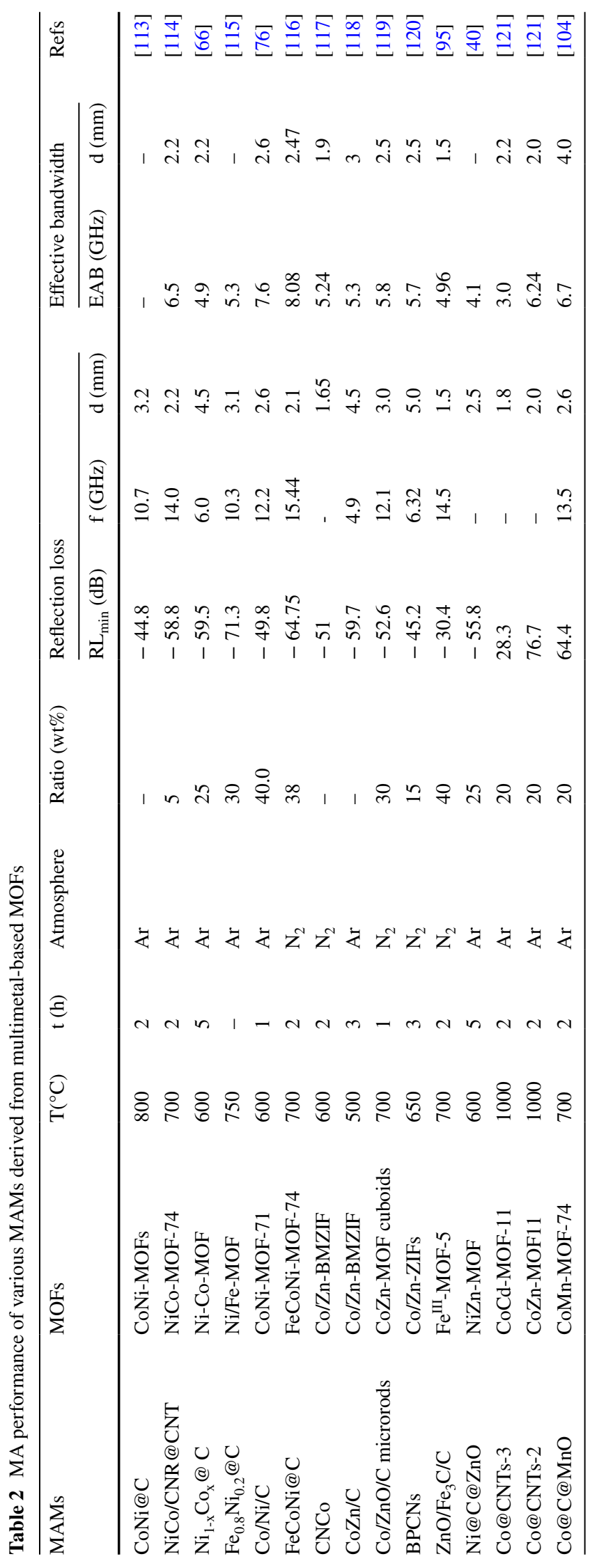



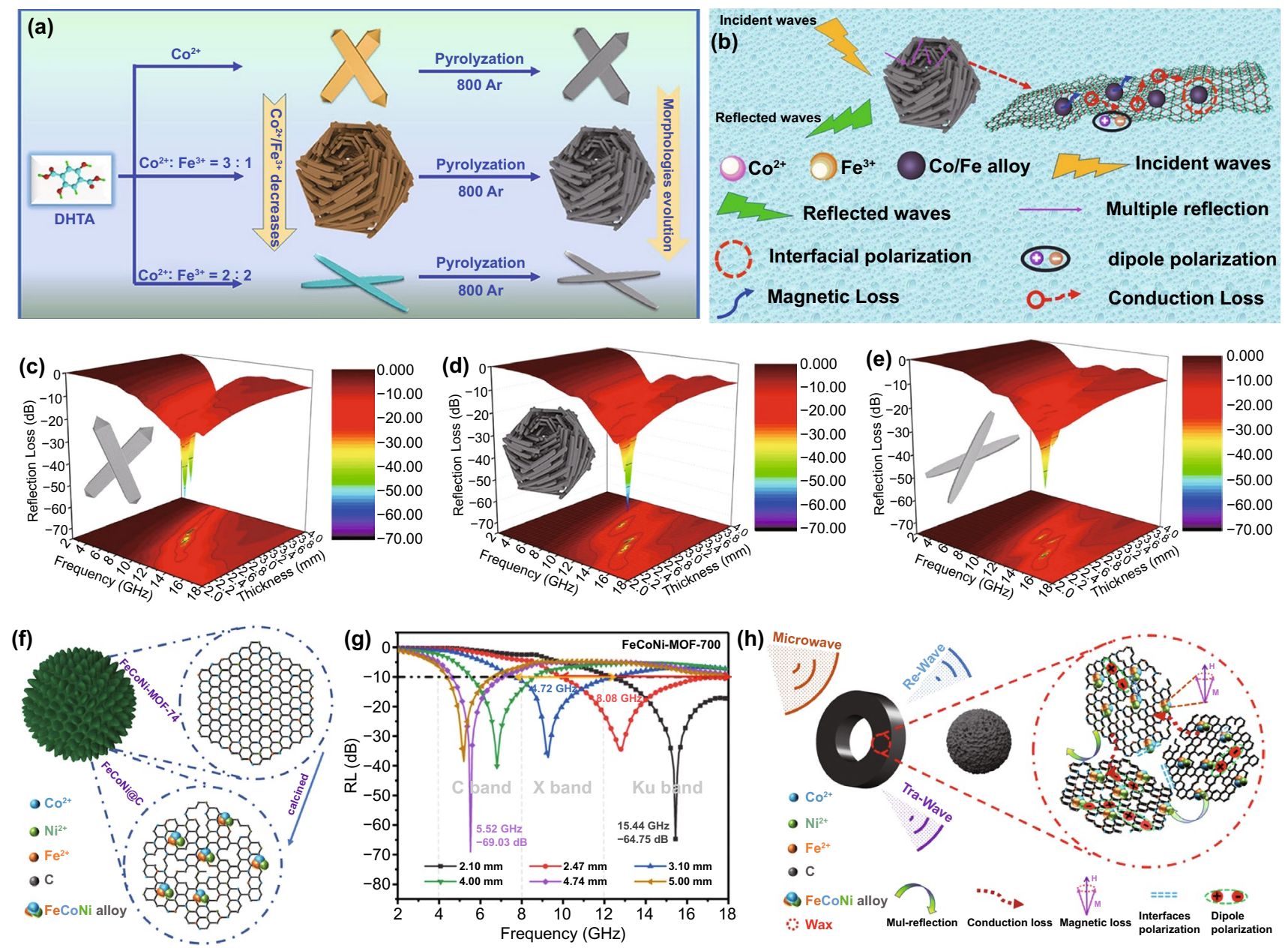

Fig. 9 Schematic illustration of a preparation process and b MA mechanism for CoFe@C composites with rod-like, nest-like and sheet-like structures. c-e 3D RL-f curves of three kinds of CoFe@C composites. Reprinted with permission from Ref. [122]. f Schematic illustration of preparation process, $\mathbf{g}$ RL-f curves and $\mathbf{h}$ MA mechanism of hollow FeCoNi@C nanocomposites. Reprinted with permission from Ref. [116]

properties of $\mathrm{FeCoNi}$ alloy. The experimental results indicated that $\mathrm{FeCoNi}$ and $\mathrm{CoFe}$ magnetic alloys coexisted in FeCoNi@C nanocomposites when the calcination temperature was $700{ }^{\circ} \mathrm{C}$, which achives the optimal performance with $\mathrm{RL}_{\min }$ of -64.75 and $-69.03 \mathrm{~dB}$ at 15.44 and $5.52 \mathrm{GHz}$, respectively (Fig. 9g). The magnetic loss of FeCoNi alloy played an important part in the MW attenuation mechanism, which enhanced the impendence matching and microwave attenuation ability (Fig. 9h).

\subsubsection{Multi-nonmagnetic Metal MOFs as Precursors}

It is worthy to attempt that we use multi-nonmagnetic metal MOFs with enhanced interfacial polarization and high porosity as precursors to prepare MAMs. Typically, Qiao and $c o$-workers prepared $\mathrm{TiO}_{2} / \mathrm{ZrTiO}_{4} /$ carbon nanocomposites using PCN-415 (TiZr-MOFs) as precursors [101]. The PCN-415 precursors were prepared through two-step solvothermal reactions, and the $\mathrm{TiO}_{2} / \mathrm{ZrTiO}_{4} /$ carbon nanocomposites were obtained after high temperature treatment (Fig. 10a). The experimental results showed that the $\mathrm{TiO}_{2} / \mathrm{ZrTiO}_{4} /$ carbon nanocomposites exhibited the best MA performance with a carbonization temperature of $800{ }^{\circ} \mathrm{C}$, and the $\mathrm{RL}_{\min }$ and $\mathrm{EAB}$ of $\mathrm{TiO}_{2} / \mathrm{ZrTiO}_{4} /$ carbon reached $-67.8 \mathrm{~dB}$ and $4.8 \mathrm{GHz}$, respectively (Fig. 10bc). For comparison, the authors fabricated $\mathrm{ZrO}_{2} / \mathrm{C}$ and $\mathrm{TiO}_{2} / \mathrm{C}$ with UIO-66 and MIL-125 as precursors under the same preparation conditions, respectively. However, the MA properties of those two MAMs were poor. The 

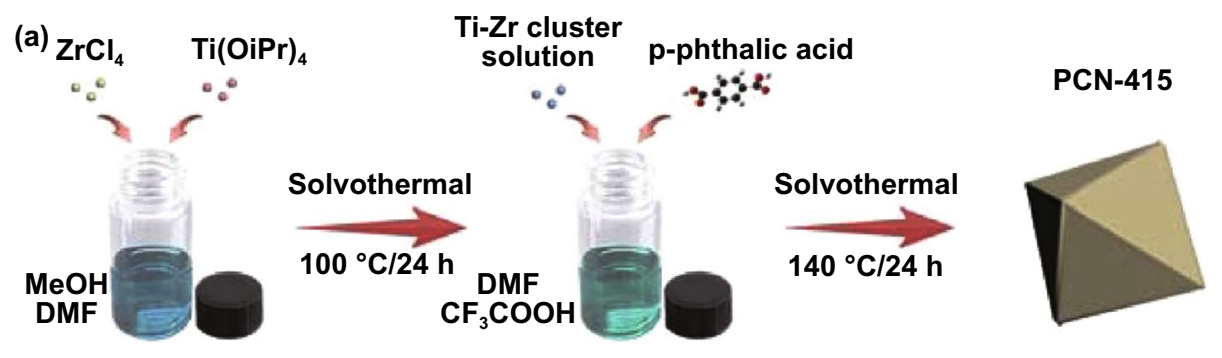

$\mathrm{TiO}_{2} / \mathrm{ZrTiO}_{4} /$ Carbon octahedral particle

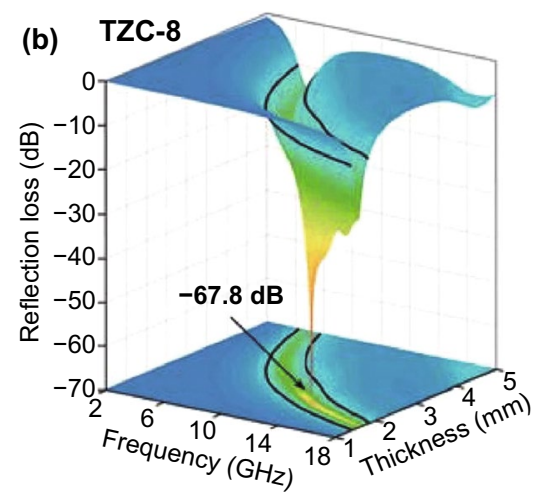

(d) Incident waves
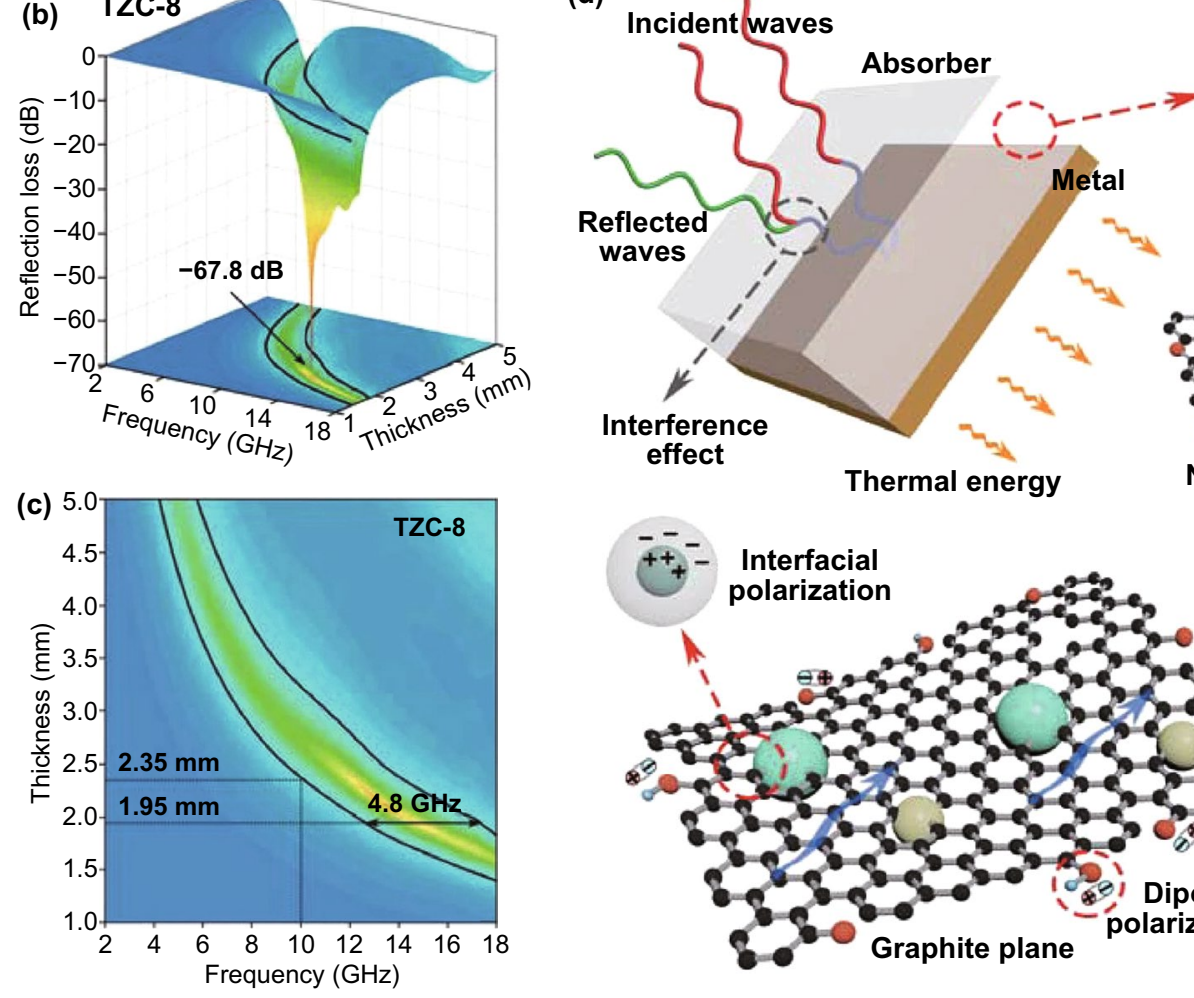

Thermal energy
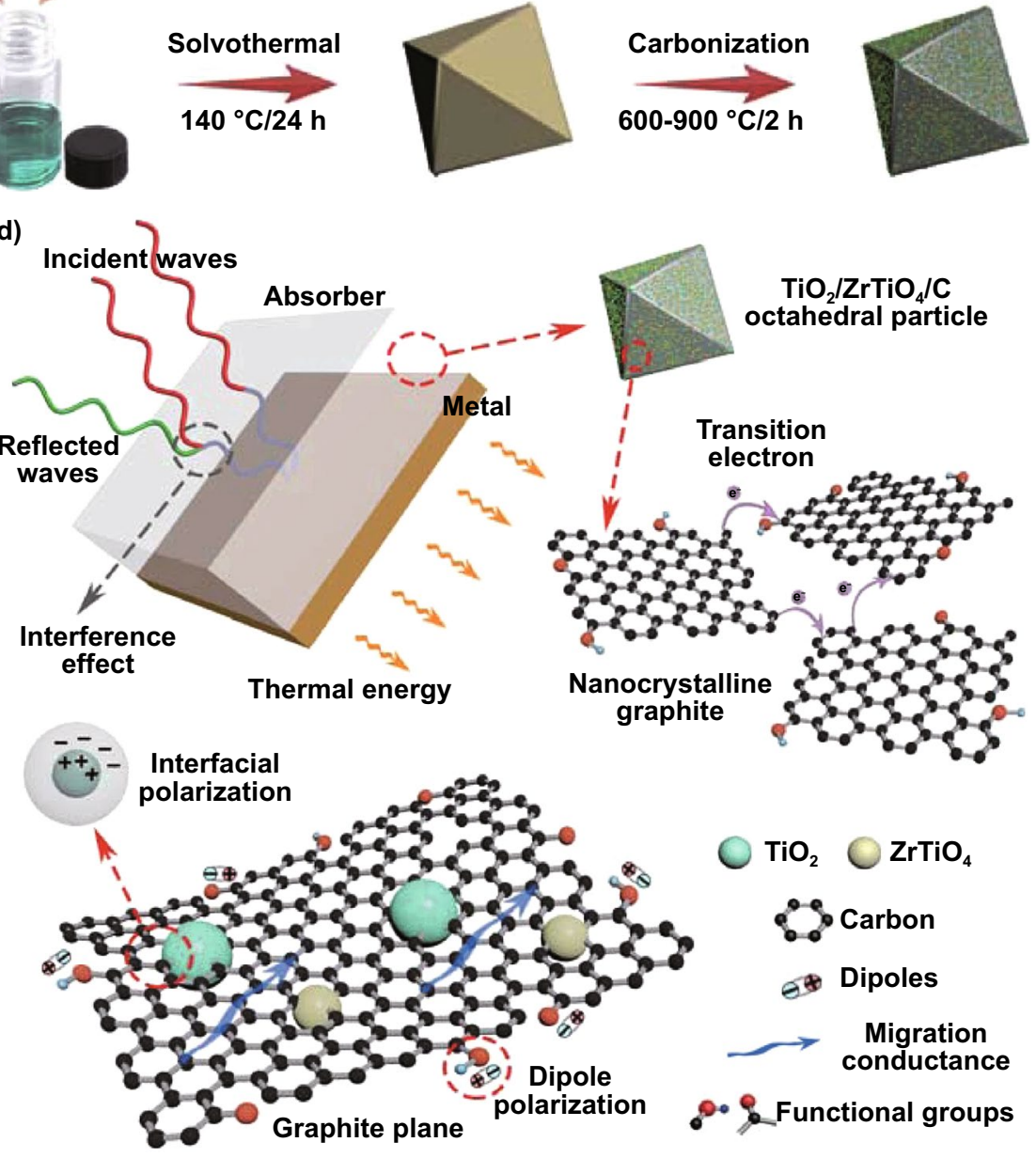

Fig. 10 a Diagrammatic sketch of the preparation. b 3D RL-f curves, $\mathbf{c}$ 2D RL projection mapping and d schematic illustration of the MA mechanism of $\mathrm{TiO}_{2} / \mathrm{ZrTiO}_{4} /$ carbon nanocomposites. Reprinted with permission from Ref. [101]

factors contributing to the above phenomenon were the effective adjustment ability for impedance matching and rich phase interfaces of $\mathrm{TiO}_{2} / \mathrm{ZrTiO}_{4} /$ carbon. The MA mechanism of $\mathrm{TiO}_{2} / \mathrm{ZrTiO}_{4} /$ carbon is depicted in Fig. 10d: firstly, the transition electron in nanocrystalline graphite provided conductive attenuation; secondly, the polarization loss originated from rich heterogeneous interfaces and functional groups further enhanced the MA property; finally, the $\mathrm{TiO}_{2}$ and $\mathrm{ZrTiO}_{4}$ optimized the natural impedance mismatching of carbon matrix, providing a necessary premise for microwave to enter $\mathrm{TiO}_{2} / \mathrm{ZrTiO}_{4} /$ carbon. More importantly, the MA ability of $\mathrm{TiO}_{2} / \mathrm{ZrTiO}_{4} /$ carbon was even better than many MAMs derived from magnetic metal
MOFs, proving the potential of multi-nonmagnetic metal MOFs in microwave absorption.

\subsubsection{Mixed Metal MOFs as Precursors}

At present, there are also many works used mixed metal MOFs as precursors of MAMs. One of the common nonmagnetic metal sources is $\mathrm{Zn}^{2+}$, which can transfer into $\mathrm{ZnO}$ with excellent dielectric properties and $\mathrm{Zn}$ which can provide plentiful pores to enhance the multiple reflection. In 2021, Wei and co-workers pyrolyzed a ZIF containing both $\mathrm{Co}$ and $\mathrm{Zn}$ to obtain CoZn alloy/carbon(CoZn/C) composites [118]. The $\mathrm{Co}^{2+}$ and $\mathrm{Zn}^{2+}$ were existed simultaneously and 

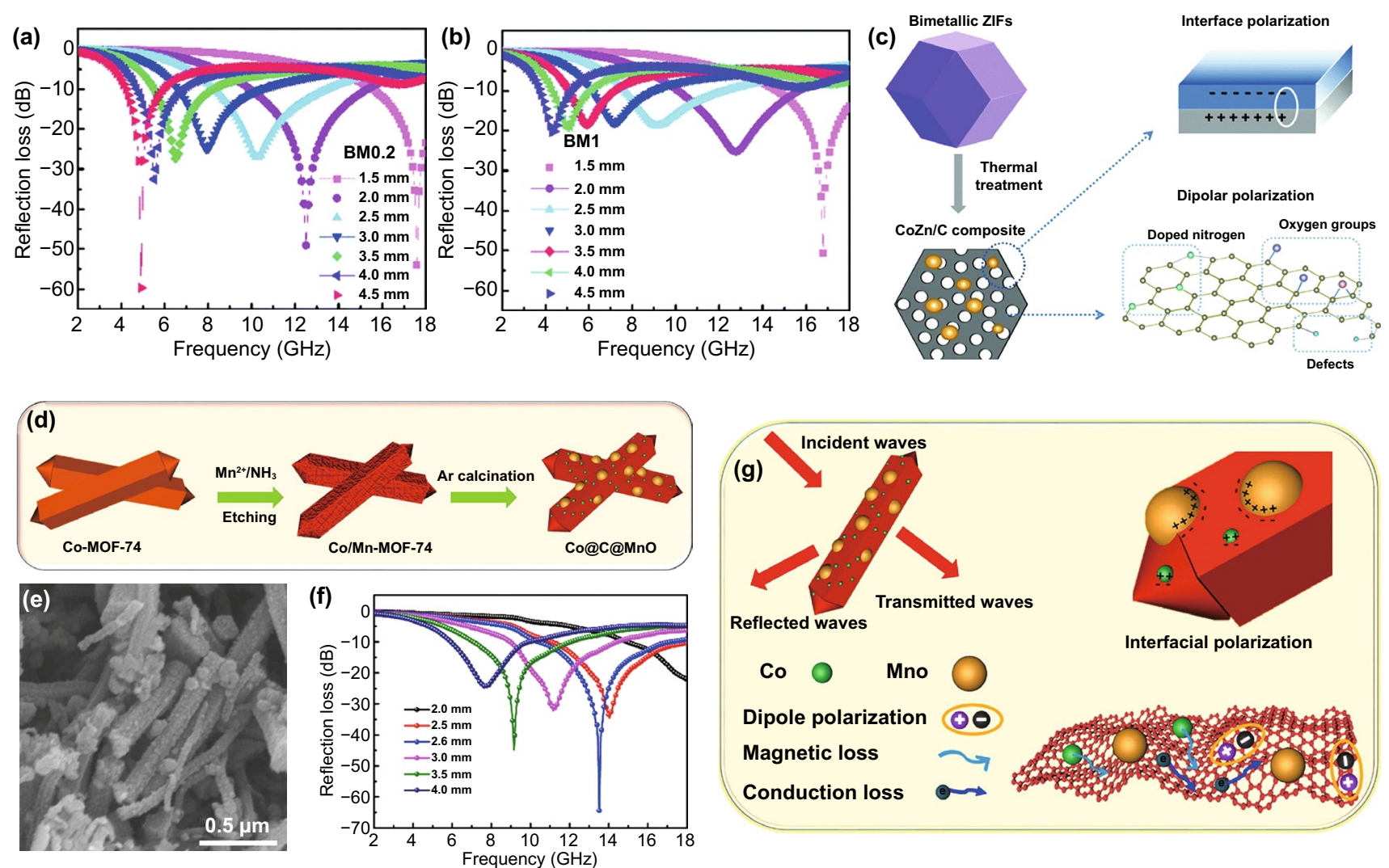

Fig. 11 RL-f curves of $\mathrm{CoZn} / \mathrm{C}$ with the $\mathrm{Zn} / \mathrm{Co}$ of a 0.2 and b 1 . c Schematic illustration of the synthesis of CoZn/C composites. Reprinted with permission from Ref. [118]. d Schematic illustration of preparation process, e SEM image, f RL-curves and $\mathbf{g}$ MA mechanism diagram of Co@C@MnO nanorod. Reprinted with permission from Ref. [104]

dispersed uniformly in this ZIF because they could coordinate with 2-methylimidazole ligands with the same structure. The graphitization, pore size distribution and surface area characteristics could be precisely controlled by adjusting the molar ratio of $\mathrm{Zn}$ to Co. Compared with ZIF-67, the pyrolysis products of bimetal ZIF presented lower pore size distribution and higher surface area because of the volatilization of $\mathrm{Zn}$. The $\mathrm{RL}_{\min }$ of the $\mathrm{CoZn} / \mathrm{C}$ composites with the $\mathrm{Zn} / \mathrm{Co}$ of 0.2 was $-59.7 \mathrm{~dB}$ and $\mathrm{Zn} / \mathrm{Co}$ of 1 was $-50.7 \mathrm{~dB}$ (Fig. 11a and b). In addition to the conductive loss and magnetic loss, the abundant heterogeneous interfaces brought about interface polarization, the doped nitrogen, oxygen groups and defects supplied sufficient dipole polarization when microwave enters the $\mathrm{CoZn} / \mathrm{C}$ composites. Besides, the abundant pores created conditions for the multiple reflections, which contributed to the MA attenuation effectively (Fig. 11c). When the pyrolysis temperature is between 550 and $800{ }^{\circ} \mathrm{C}$, the zinc element in zinc-based MOF mainly exists in the form of $\mathrm{ZnO}$. Wang designed a yolk-shell
$\mathrm{Ni@C@ZnO} \mathrm{microsphere} \mathrm{with} \mathrm{an} \mathrm{open} \mathrm{gap} \mathrm{between} \mathrm{the}$ core and shell by directly calcining Ni-Zn-MOF [40]. The $\mathrm{RL}_{\min }$ of $\mathrm{Ni} @ \mathrm{C} @ \mathrm{ZnO}$ reached $-55.0 \mathrm{~dB}$ and the EAB covered $4.1 \mathrm{GHz}$, which could attribute to the increasing interface polarization and optimization of impedance matching.

In order to increase interfacial polarization, dipole polarization or optimize impedance matching, some other metal ions have also been attempted to be used in the center of MOF together with magnetic metal ions to prepare precursors of high-performance MAMs. Qiu et al. fabricated CoMn-MOF-74 nanomaterials by the etching method [104]. The $\mathrm{Mn}^{2+}$ coordinated with the ligands in Co-MOF-74 to form CoMn-MOF-74, and the Co@C@MnO nanorods were obtained after calcination of CoMn-MOF-74 (Fig. 11d-e). The $\mathrm{RL}_{\min }$ was $-61.8 \mathrm{~dB}$ at $13.5 \mathrm{GHz}$, and the EAB was $6.7 \mathrm{GHz}$ (Fig. 11f). The Co nanoparticles were uniformly distributed in the carbon matrix, which could produce strong magnetic loss and conductive loss. Besides, there were many interfaces between $\mathrm{Co}, \mathrm{C}$ and $\mathrm{MnO}$, effectively enhancing 
the interface polarization (Fig. 11g). In 2021, Zhang and co-workers synthesized a novel $\mathrm{Co} / \mathrm{CoO} / \mathrm{SrCO}_{3} @ \mathrm{C}$ nanocomposite using CoSr-bimetal MOF as precursor [124]. This product exhibited great MA performance with $\mathrm{RL}_{\text {min }}$ of $-39.51 \mathrm{~dB}$ at $2 \mathrm{~mm}$ and $\mathrm{EAB}$ of $2.55 \mathrm{GHz}$ at a relatively thin thickness of $1 \mathrm{~mm}$, in which the interwoven nanoparticles and hollow nanotubes played a significant part on the MA progress.

\section{MAMs Derived from MOF Composites}

To further optimize the electromagnetic parameters of MAMs, combining MOFs with other materials with great electrical or magnetic conductivity as carbonized precursors is also a popular method. Compared with pure MOFs as precursors, the preparation process of MOF composites is slightly more complex, but it can adjust the electromagnetic parameters more specifically and effective. There are three main methods to form MOF composites: (i) introducing magnetic nanoparticles, (ii) constructing MOF and lowdimensional material hybrids and (iii) designing core-shell structure. In this section, three methods are introduced to deeply understand the mechanism of microwave absorption to guide the future relative research.

\subsection{Introducing Magnetic Nanoparticles}

The impedance mismatch is a problem to face for many MOF-derived MAMs. An effective way to solve the problem is increasing magnetic nanoparticles of MAMs to improve the magnetic loss capacity. There are two ways to achieve this goal: incorporating magnetic nanoparticles into MOF and mixing metal salts with precursor solution of MOF. In Table 3, we make a list of the MA performance of the MAMs as mentioned above.

\subsubsection{Doping Magnetic Nanoparticles}

To improve the magnetic property of MAMs, incorporating magnetic metal nanoparticles (NPs) or metallic oxide into MOF precursors is a direct method. In 2015, a novel Co-Fe/ NPC nanomaterial was prepared by the thermal decomposition of $\mathrm{Fe}_{3} \mathrm{O}_{4}$ NP-modified ZIF-67 (IONP@ZIF-67) [128]. Although the preparation process of $\mathrm{Fe}-\mathrm{Co} / \mathrm{NPC}$ was relatively complex, including the preparation of $\mathrm{Fe}_{3} \mathrm{O}_{4} \mathrm{NPs}$,

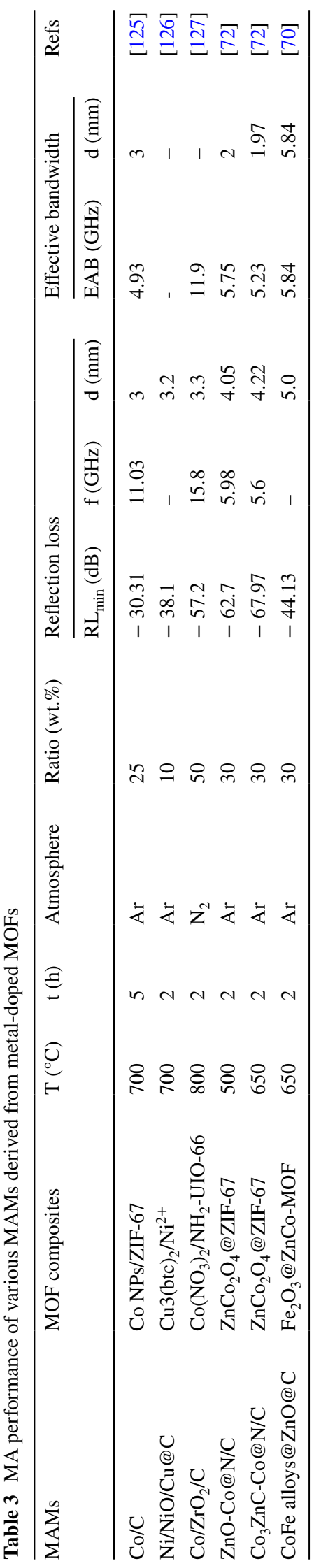



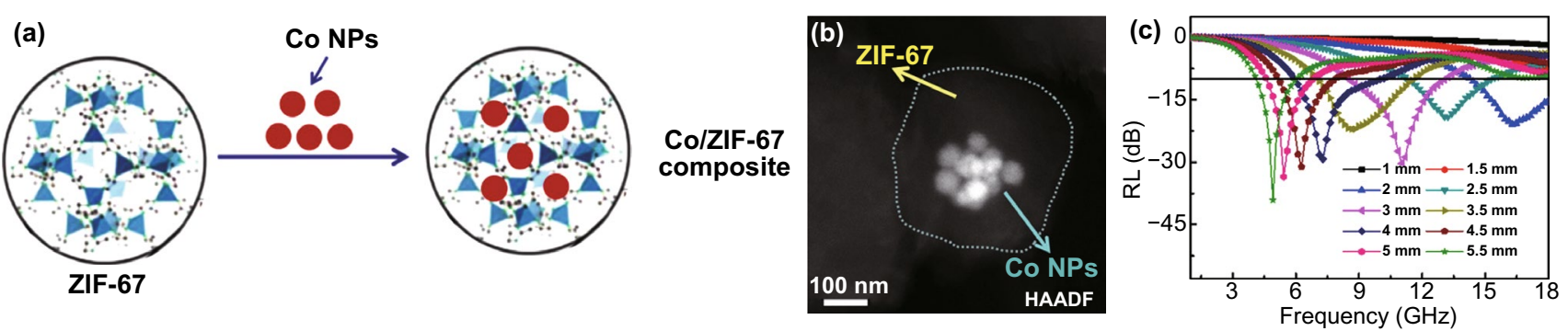

(d)

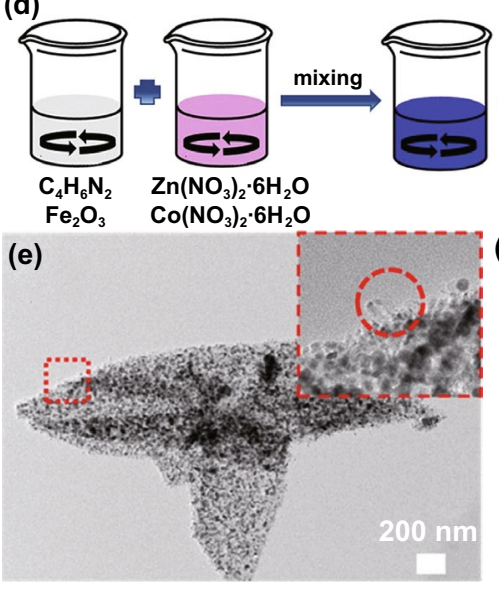

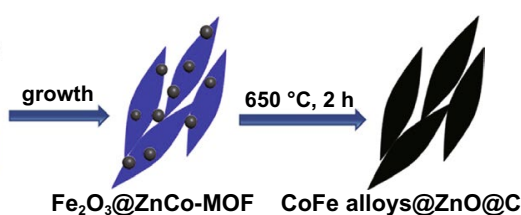

(g)

Thermal wave
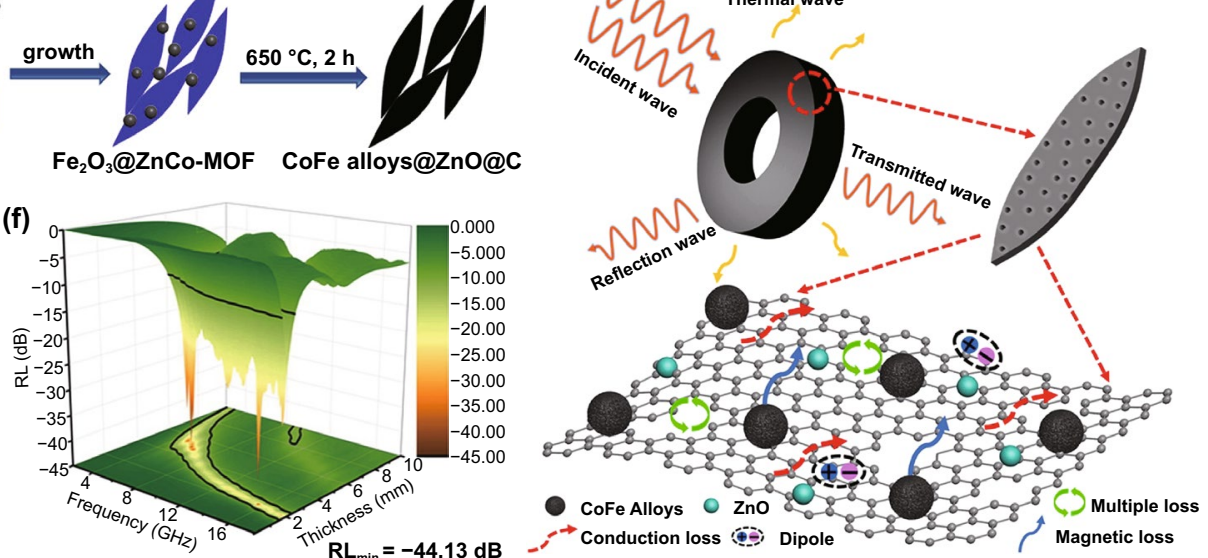

Fig. 12 a Schematic diagram of the fabrication and b HAADF image of Co NPs/ZIF-67, c RL-f curves of Co/C nanocomposites. Reprinted with permission from Ref. [125]. d Schematic illustration of experimental procedures e TEM and $\mathbf{f}$ RL-f curves of CoFe alloys@ZnO@C composites. g Schematic illustration of the MA mechanism of CoFe alloys@ZnO@C. Reprinted with permission from Ref. [70]

encapsulation of $\mathrm{Fe}_{3} \mathrm{O}_{4}$ with DNAA, and the synthesis of IONP@ZIF-67 and Fe-Co/NPC, the MA performance of $\mathrm{Fe}-\mathrm{Co} / \mathrm{NPC}$ nanomaterials was quite good with $\mathrm{RL}_{\min }$ of $-21.7 \mathrm{~dB}$ with a thickness of $1.2 \mathrm{~mm}$ and a broad EAB of 5.8 GHz. Liu embedded Co NPs into ZIF-67 to act as the precursors of $\mathrm{Co} / \mathrm{C}$ nanocomposites (Fig. 12a) [125]. Co NPs were firstly modified with PVP and then mixed with $\mathrm{Co}\left(\mathrm{NO}_{3}\right)_{2}$ to produce Co NPs/ZIF-67. As shown in Fig. 12b, the Co NPs/ZIF-67 maintained the dodecahedron structure and the Co NPs were fully encapsulated in ZIF-67. The $\mathrm{Co} / \mathrm{C}$ nanocomposites shown improved impedance matching and thus an excellent MA ability with $\mathrm{RL}_{\min }$ of $-30.31 \mathrm{~dB}$ at $11.03 \mathrm{GHz}$ and EAB of $4.93 \mathrm{GHz}$ (Fig. 12c). Similarly, Wu's group synthesized CoFe alloys@ZnO@C composites by directly carbonizing $\mathrm{Fe}_{2} \mathrm{O}_{3} @ \mathrm{ZnCo}-\mathrm{MOF}$ at $650{ }^{\circ} \mathrm{C}$ (Fig. 12d) [70]. The obtained $\mathrm{CoFe}$ alloys and $\mathrm{ZnO}$ nanocomposites were evenly distributed in carbon matrix, and some carbon nanotubes appeared in the surface (Fig. 12e). The optimized $\mathrm{RL}_{\min }$ achieved $-40.63 \mathrm{~dB}$ at a thickness of $2.2 \mathrm{~mm}$ and $-44.13 \mathrm{~dB}$ at $5.0 \mathrm{~mm}$ (Fig. 12f). The CoFe alloys mainly contributed magnetic loss of this MAM, and the presence of $\mathrm{ZnO}$ balanced the high conductivity of graphitized carbon and than improved the impedance matching performance. Besides, the heterogeneous interface, defects and polarization groups also greatly contributed to the attenuation of microwave (Fig. 12g).

\subsubsection{Mixing Metal Salts with Precursors Solution of MOF}

Compared with directly doping magnetic nanoparticles, mixing metal ions with precursor solution of MOFs is more simple and efficient method to optimize magnetic loss and further promote the impedance matching. Yu prepared a novel $\mathrm{Fe}-\mathrm{N} / \mathrm{C}$ nanocomposite using Fe@ZIF-8 as precursor through one-pot method [129], in which the phthalocyanine (FePc) was added simultaneously with 2-MeIm and $\mathrm{Zn}^{2+}$ to in situ get Fe@ZIF-8 composite, which was followed by the hightemperature calcination to obtain Fe-N/C (Fig. 13a). During the pyrolysis process, the $\mathrm{Zn}^{2+}$ transformed into $\mathrm{Zn} \mathrm{NPs}$ and 
(a)

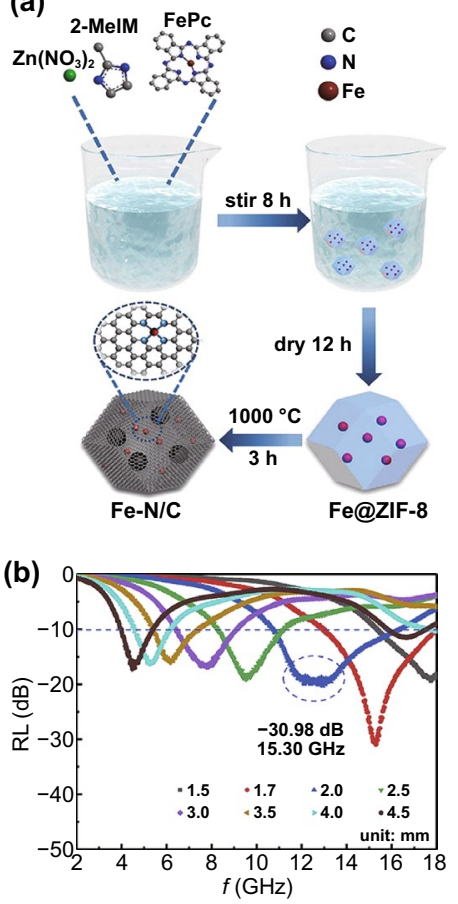

(g)

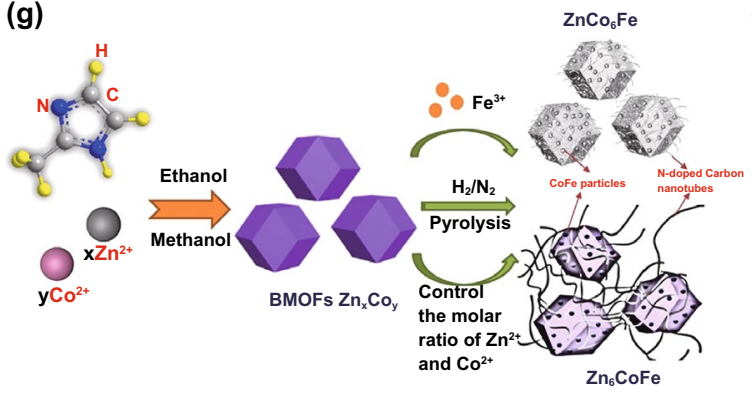

(c)

(d)

(e)
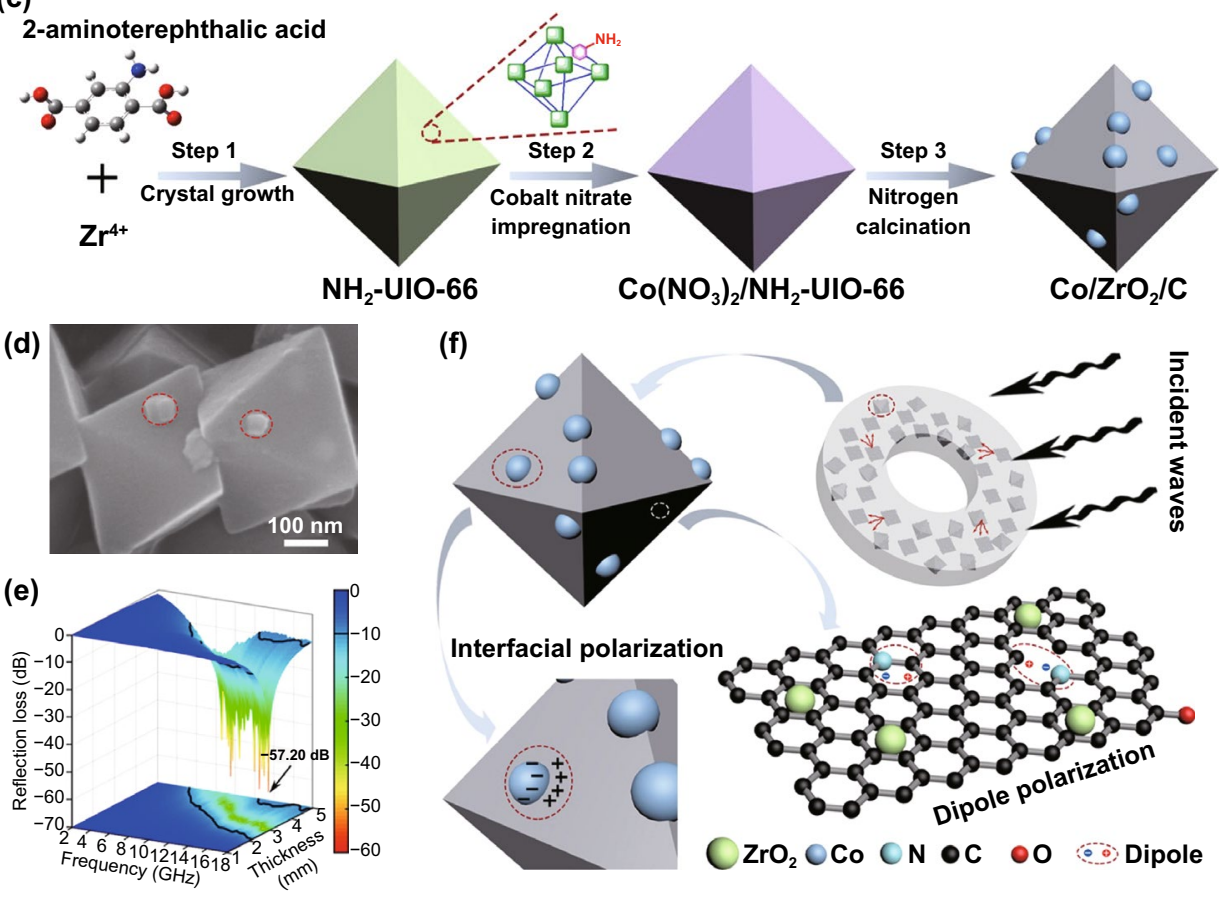

(f)
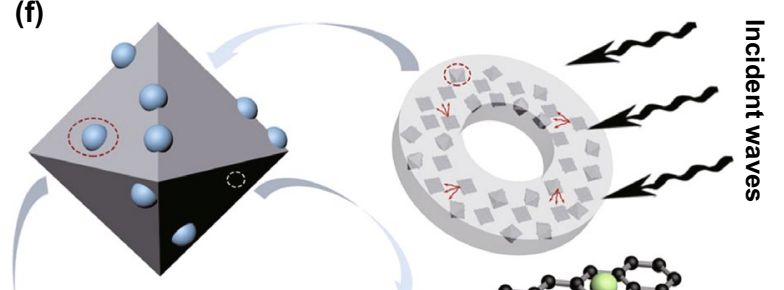

Interfacial polarization
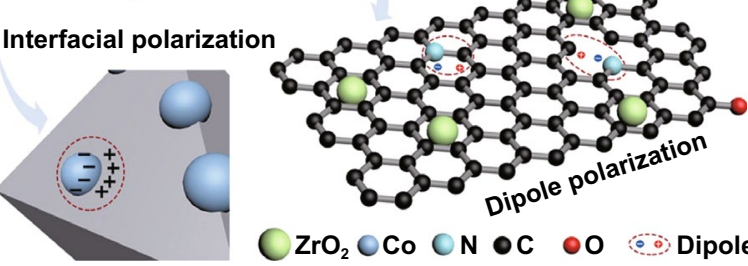

$\mathrm{ZrO}_{2}$ OCo ON oC $0 \mathrm{O}$ Dipole (h)

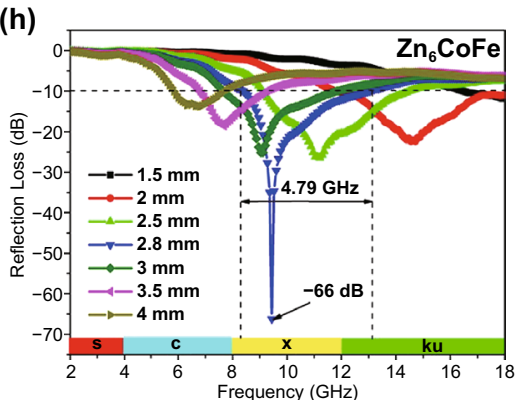

(i)

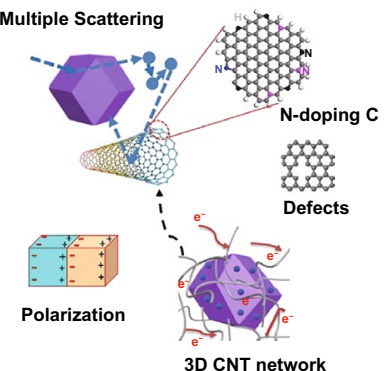

Fig. 13 a Diagrammatic sketch of the synthesis and b RL-f curves of Fe-N/C. Reprinted with permission from Ref. [129]. c Schematic illustration of the preparation, $\mathbf{d}$ SEM image, e 3D RL-f curves, $\mathbf{f}$ MA mechanism of $\mathrm{Co} / \mathrm{ZrO}_{2} / \mathrm{C}$ octahedrons. Reprinted with permission from Ref. [127]. g Schematic diagram of synthesis, $\mathbf{h}$ RL-f curves and $\mathbf{i}$ schematic representation of MA mechanism of $\mathrm{Zn}_{\mathrm{x}} \mathrm{Co}_{\mathrm{y}} \mathrm{Fe} \mathrm{C} @ \mathrm{CNTs}$. Reprinted with permission from Ref. [130]

then vapored, whereas the $\mathrm{Fe}^{2+}$ was reduced to Fe NPs. The $\mathrm{Fe}-\mathrm{N} / \mathrm{C}$ achieved a MA property with the optimized $\mathrm{RL}_{\min }$ of $-30.98 \mathrm{~dB}$ at a low thickness of $1.7 \mathrm{~mm}$ (Fig. 13b).

The central atoms of electron-donating groups (such as $-\mathrm{NH}_{2}$ and $-\mathrm{COOH}$ ) are electronegative, which can effectively capture the metal ions and form the coordination bonds. Zhang successfully synthesized $\mathrm{Co} / \mathrm{ZrO}_{2} / \mathrm{C}$ octahedrons by the carbonization of $\mathrm{Co}\left(\mathrm{NO}_{3}\right)_{2}$-impregnated $\mathrm{NH}_{2}$-UIO-66 [127]. The $\mathrm{Co}^{2+}$ was coordinated with $\mathrm{N}$ of $-\mathrm{NH}_{2}$ to form $\mathrm{Co}\left(\mathrm{NO}_{3}\right)_{2} / \mathrm{NH}_{2}$-UIO-66; the pyrolysis product displayed the primitive morphology with Co NPs embedded in the surface (Fig. 13c-d). The $\mathrm{Co} / \mathrm{ZrO}_{2} / \mathrm{C}$ possessed the excellent MA performance with $\mathrm{RL}_{\text {min }}$ of $-57.2 \mathrm{~dB}$ at $15.8 \mathrm{GHz}$ and $\mathrm{EAB}$ covered as wide as $6.5 \mathrm{GHz}$ (Fig. 13e). The introduced Co NPs provided the magnetic loss to enhance the impendence matching and attenuation capacity. Besides, the number of heterogeneous interfaces, defects and polarization groups, which benefited to polarization loss, was also improved (Fig. 13f).

Besides, solution infiltration is also a common approach to add metal ions into MOFs. The metal ions could permeate into the channel of MOFs through the capillary force at 
the orifice and the metal nanoparticles would be embedded into carbon matrix after pyrolysis. In 2020, Yan impregnated the $\mathrm{CoZn-MOF}$ precursors with $\mathrm{FeCl}_{3}$ solutions; the corresponding $\mathrm{Zn}_{\mathrm{x}} \mathrm{Co}_{\mathrm{y}} \mathrm{Fe} @ \mathrm{C} @ \mathrm{CNTs}$ were obtained after calcination in the mixture of $\mathrm{H}_{2}$ and $\mathrm{N}_{2}$ (Fig. 13g) [130]. In the heating process, the cavity could be generated because of the volatilization of $\mathrm{Zn}$, which promoted the encapsulation of Fe to form CoFe alloy and catalyzed the growth of CNTs. As shown in Fig. 13h, the $\mathrm{RL}_{\min }$ of $\mathrm{Zn}_{\mathrm{x}} \mathrm{Co}_{\mathrm{y}} \mathrm{Fe} @ \mathrm{C} @ \mathrm{CNTs}$ reached $-66 \mathrm{~dB}$ and the $\mathrm{EAB}$ covered the whole $\mathrm{X}$ band. The great MA performance originated from the multiply scattering, polarization loss originated from $\mathrm{N}$-doping $\mathrm{C}$, defects and many interfaces, conductive loss of 3D CNT networks and the magnetic loss of CoFe alloy (Fig. 13i). In 2019, Huang and $c o$-workers prepared $\mathrm{Ni} / \mathrm{NiO} / \mathrm{Cu} @ \mathrm{C}$ nanocomposites using $\mathrm{Cu}_{3}(\mathrm{btc})_{2} / \mathrm{Ni}^{2+}$ as precursors [126]. The $\mathrm{Ni}^{2+}$ was ground into the ordered pores of $\mathrm{Cu}_{3}(\mathrm{btc})_{2}$ with assist of ethanol flow, resulting in the incorporation of Ni NPs into the $\mathrm{Cu} @ \mathrm{C}$ nanocomposites to produce NiO NPs on the surface after the high-temperature calcination. Compared with $\mathrm{Cu} @ \mathrm{C}$ nanocomposites, the MA property of $\mathrm{Ni} / \mathrm{NiO}$ / $\mathrm{Cu} @ \mathrm{C}$ was obviously improved with $\mathrm{RL}_{\min }$ of $-38.1 \mathrm{~dB}$ at $3.2 \mathrm{~mm}$ when the filler loading of $\mathrm{Ni} / \mathrm{NiO} / \mathrm{Cu} @ \mathrm{C}$ in paraffin matrix was merely $10 \mathrm{wt} \%$.

\subsection{Loading on Low-Dimensional Materials}

Generally, the ideal MAMs should have conductive networks with good conductive loss; however, most MOF-derived MAMs only can possess good conductivity rather than conductive networks, which seriously limit their further application in MA field. Therefore, designing MOF-derived MAMs containing low-dimensional conductive carbon materials and porous magnetic component is considered as an efficient approach to solve the above issue. The conductivity of the composites can be improved and the surface electron transfer also could be accelerated. Meanwhile, the defects can be generated at the interface and surface, resulting in the increase in dielectric loss. Generally, high graphitization materials can be obtained by the high-temperature treatment of some carbon-rich materials such as conductive carbon materials, biomaterials, $\mathrm{MXene}$ and $\mathrm{g}-\mathrm{C}_{3} \mathrm{~N}_{4}$. Besides, some other materials with large specific surface area, electrical or dielectric properties are also worth to try. A series of works published in the area of MAMs derived from MOF hybrids with low-dimensional materials are presented in Table 4.

\subsubsection{Loading on Conductive Carbon Materials}

Common conductive carbon materials include graphene oxide (GO), carbon nanotube (CNT), carbon nanofiber (CNF), etc., which are considered to the ideal template for constructing lightweight MAMs due to their high strength, large specific surface area and high conductivity [139-141]. There are a large number of active sites (such as - $\mathrm{OH}$ and-COOH groups) in the surface of GO and CNT, which can absorb metal ions through electrostatic interaction to form MOFs in situ. For instance, Zhang and co-workers produced Co/C-rGO composites by calcining GO-wrapped ZIF-67 (Co@ZIF-67) at high temperature [133]. The corresponding $\mathrm{RL}_{\min }$ reached $-52 \mathrm{~dB}$ at $9.6 \mathrm{GHz}$, and the EAB was $7.72 \mathrm{GHz}$. Xu proposed a novel method by using CoNi-BTC/rGO as precursors to produce pomegranate-like CoNi@NC/rGO-600 nanocomposites [49], in which the CoNi@NC/rGO-600 exhibited outstanding MA performance with a broad $\mathrm{EAB}$ of $6.7 \mathrm{GHz}$ and a $\mathrm{RL}_{\min }$ of $-68.0 \mathrm{~dB}$. The authors also served CoNi@NC-600 derived from CoNi-BTC as a comparative reference, but its MA property was poorer than that of $\mathrm{CoNi} @ \mathrm{NC} / \mathrm{rGO}-600$ obtained in the same condition; the main reason for this phenomenon was that $\mathrm{GO}$ provided a smooth conductive network.

In 2021, Fang and co-workers loaded ZIF-67 on CNTs to serve as the precursors of hierarchical Co@C/CNTs [135]. As shown in Fig. 14a, the $\mathrm{Co}^{2+}$ in ZIF-67 mainly be reduced to Co nanoparticles during the pyrolysis process, and a little part were oxidized to $\mathrm{Co}_{3} \mathrm{O}_{4}$. The experimental results revealed that the as-prepared $\mathrm{Co@C/CNTs} \mathrm{exhibited}$ strong MA performance both at room temperature and low temperature of $40{ }^{\circ} \mathrm{C}$. The $\mathrm{RL}_{\text {min }}$ was achieved $-57 \mathrm{~dB}$ at $4.5 \mathrm{~mm}$ and $-30 \mathrm{~dB}$ at a thin thickness of $1.5 \mathrm{~mm}$ (Fig. 14b), in which dielectric loss and magnetic loss were provided by the interconnected CNT network and Co-contained magnetic-particles, respectively. Actually, the morphology also plays a key role in MA performance of MAMs (Fig. 14c). In 2020, the effect of the introduction of different dimensional carbon ( $\mathrm{rGO}$ or CNT) matrix was studied by Wang and co-workers. They synthesized rGO-CoFe@C 


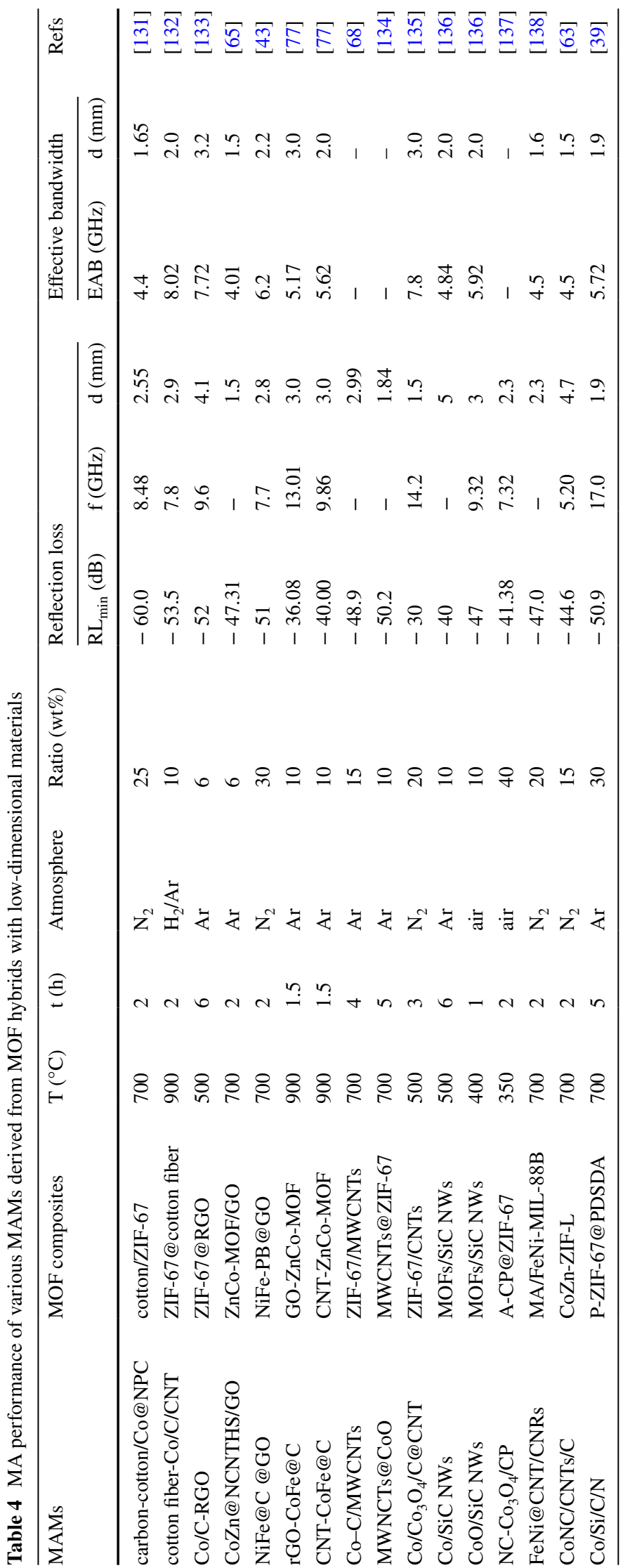


(a)
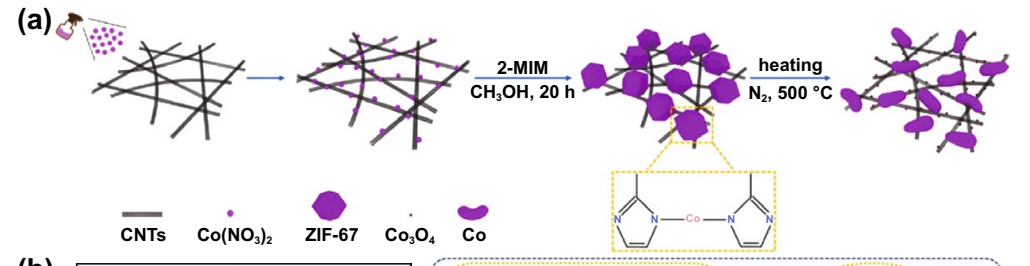

(b)

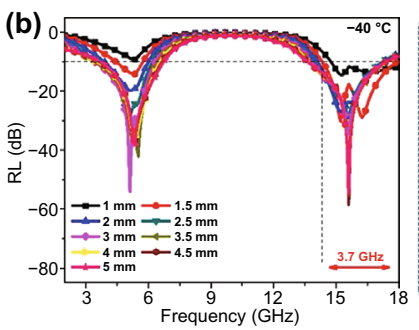

(e)
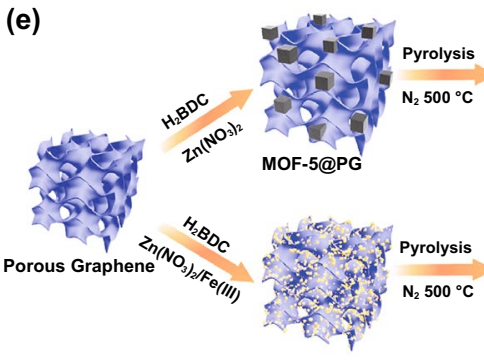

$\mathrm{Fe}(\mathrm{III} / \mathrm{MOF-5@PG}$

(f)

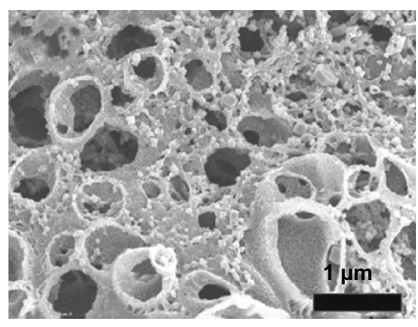

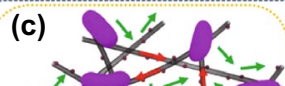
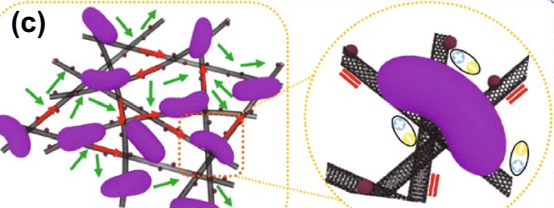

$\longrightarrow$ Multi-scattering and multi-reflection $\longrightarrow$ Conductive loss

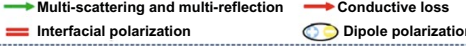

(h)
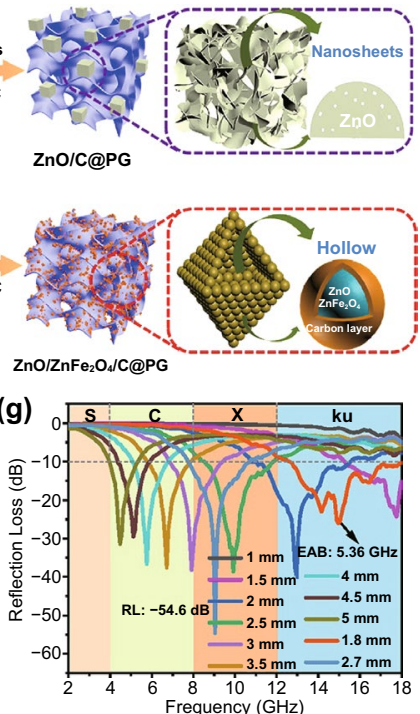

(d)
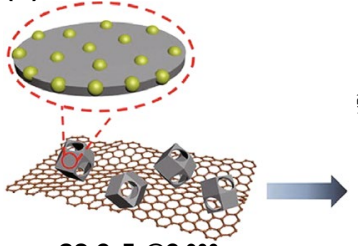

rGo-CoFe@C-900
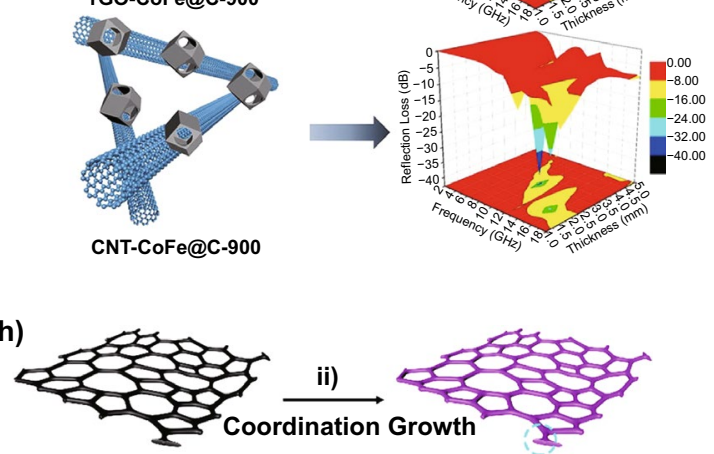
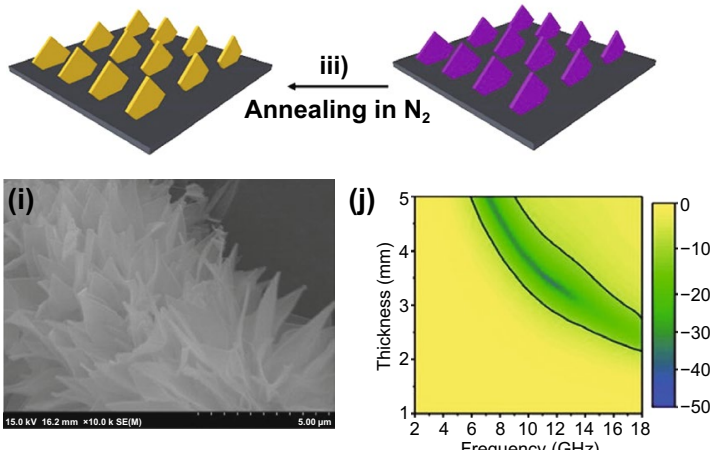

Fig. 14 a Schematic illustration of the synthesis b RL-f curves and $\mathbf{c}$ MA mechanism of Co-based MOF-derived carbon/CNTs. Reprinted with permission from Ref. [135]. d Schematic illustration of preparation and RL-f curves of rGO-CoFe@C and CNT-CoFe@C. Reprinted with permission from Ref. [77]. e Schematic illustration of the synthesis of $\mathrm{ZnO} / \mathrm{C} @ \mathrm{PG}$ and $\mathrm{ZnO} / \mathrm{ZnFe}_{2} \mathrm{O}_{4} / \mathrm{C} @ \mathrm{PG}$. $\mathbf{f}$ SEM image and $\mathbf{g} \mathrm{RL}-\mathrm{f}$ curves of $\mathrm{ZnO} / \mathrm{ZnFe}_{2} \mathrm{O}_{4} / \mathrm{C} @ \mathrm{PG}$. Reprinted with permission from Ref. [45]. h Schematic illustration the synthesis. i SEM image and $\mathbf{j} 2 \mathrm{D}$ RL projection mapping of $\mathrm{Co}_{3} \mathrm{O}_{4} / \mathrm{CF}$. Reprinted with permission from Ref. [142]

and CNT-CoFe@C nanocomposites using GO and CNT as carriers to grow $\mathrm{ZnCo} \mathrm{MOF}$ in situ, following by embedding $\mathrm{Fe}^{3+}$ in the pores of $\mathrm{ZnCo}-\mathrm{MOF}$ and calcining under $\mathrm{Ar}$ atmosphere (Fig. 14d) [77]. The $\mathrm{RL}_{\min }$ and EAB of rGOCoFe@C reached $-36.08 \mathrm{~dB}$ and $5.17 \mathrm{GHz}$, respectively. However, CNT-CoFe@C had a better performance with $\mathrm{RL}_{\text {min }}$ up to $-40.00 \mathrm{~dB}$ and $\mathrm{EAB}$ of $5.62 \mathrm{GHz}$. The reasons for this phenomenon were that CNTs that owned high aspect ratio and the $\mathrm{P}$ electrons in carbon atoms were conjugated to form large $\pi$ bonds, which made it easier to form threedimensional conductive network than rGO.
In addition to one-dimensional and two-dimensional materials, three-dimensional materials also have great application potential in the field of microwave absorption due to their porous structure, interconnected networks and abundant interfaces. Song et al. used ingenious three-dimensional porous graphene (PG) as template to grow MOF-5 and Fe(III)MOF-5 and treated at high-temperature to prepare ZnO/C@ $\mathrm{PG}$ and $\mathrm{ZnO} / \mathrm{ZnFe}_{2} \mathrm{O}_{4} / \mathrm{C} @ \mathrm{PG}$ materials (Fig. 14e) [45]. The pyrolysis products still maintained the nanoscale hollow structure, which increases the propagation path of electromagnetic wave in the MAM (Fig. 14f). Furthermore, $\mathrm{ZnO} / \mathrm{ZnFe}_{2} \mathrm{O}_{4} / \mathrm{C} @$ $\mathrm{PG}$ had a stronger magnetic loss than $\mathrm{ZnO} / \mathrm{C} @ \mathrm{PG}$ due to the 
(a)
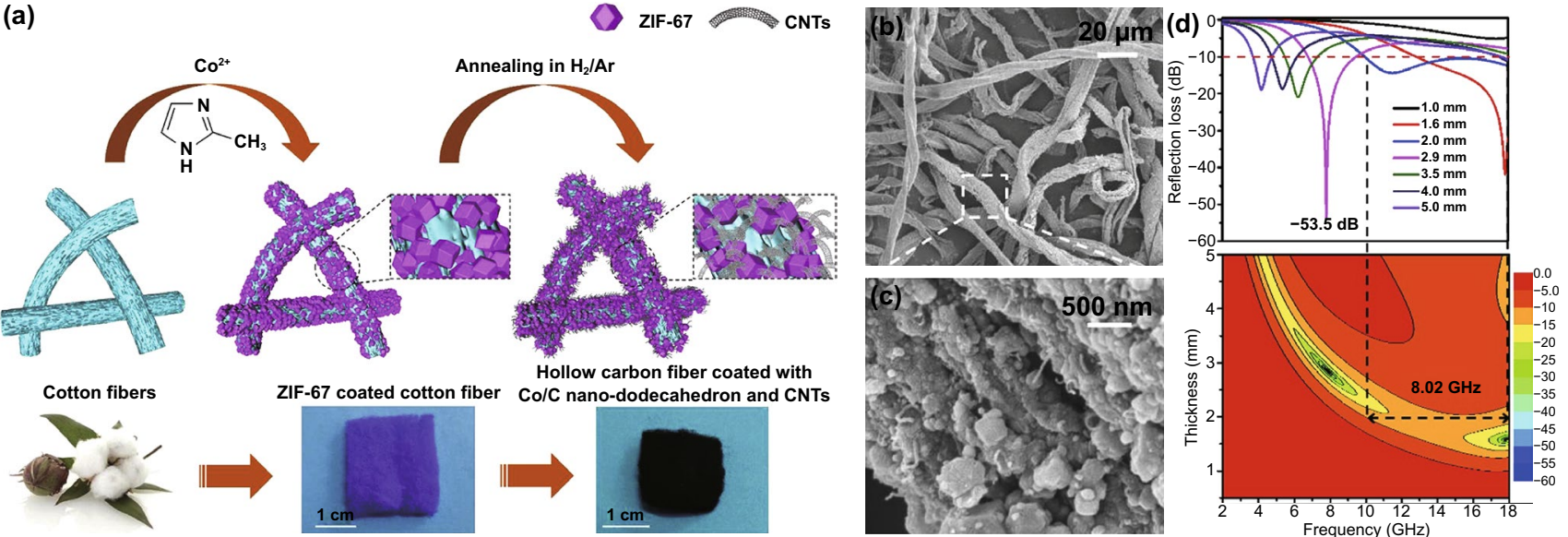

(e)
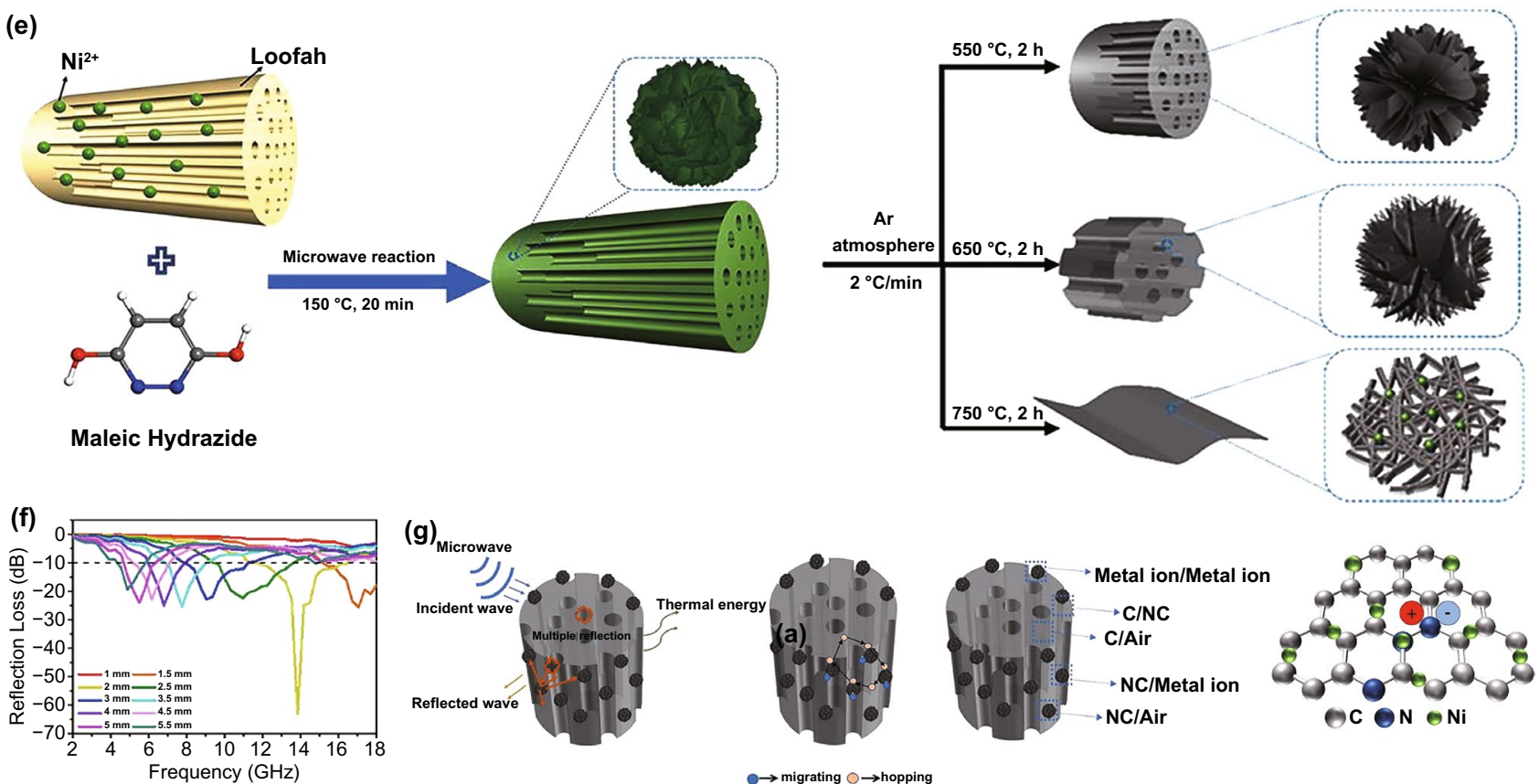

Fig. 15 a Schematic illustration of the preparation b, $\mathbf{c}$ SEM images and d RL-f curves and corresponding 2D RL-f mapping of carbon fiber/ Co@C/CNTs. Reprinted with permission from Ref. [132]. e Schematic illustration of the synthesis process of Ni/NC/C-T composites. f RL-f curves and $\mathbf{g}$ MA mechanism of Ni/NC/C-T-650. Reprinted with permission from Ref. [146]

introduced $\mathrm{Fe}(\mathrm{III})$, so that exhibited more impressive MA performance with a $\mathrm{RL}_{\min }$ of $-54.6 \mathrm{~dB}$ at $9.04 \mathrm{GHz}$ and $\mathrm{EAB}$ of $5.36 \mathrm{GHz}$ (Fig. 14g). Liu' group loaded ZIF-67 on the surface of lightweight carbon foam (CF) to act as the precursors of $\mathrm{Co}_{3} \mathrm{O}_{4} / \mathrm{CF}$ (Fig. 14h) [142]. During the high-temperature pyrolysis process, the $\mathrm{Co}^{2+}$ transferred into $\mathrm{Co}_{3} \mathrm{O}_{4}$ sheet embedded on the carbon matrix, which benefited the dual electromagnetic energy attenuation mechanisms (Fig. 14i). The $\mathrm{RL}_{\text {min }}$ of $\mathrm{Co}_{3} \mathrm{O}_{4} / \mathrm{CF}$ was $-46.58 \mathrm{~dB}$ at $10.72 \mathrm{GHz}$, and the EAB was as broad as $5.4 \mathrm{GHz}$ (Fig. 14j).

\subsubsection{Loading on Biomaterials}

Due to the environmental friendliness, easy availability and low cost, many natural biomaterials have been used as carbon sources to prepare ultra-lightweight high-performance MAMs in recent years [143-145]. Despite their high dielectric constant, the biomaterial-derived porous carbon materials are lack of magnetic loss system and cannot achieve appropriate impendence matching [145]. Researchers often recombine biomaterials with other functional materials, especially magnetic materials, to explore superior MA 
(a)

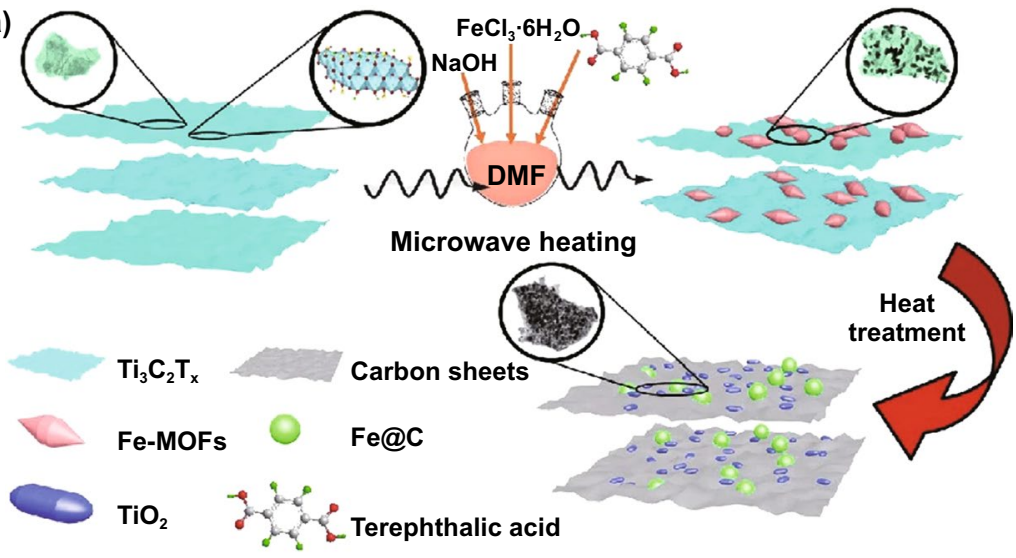

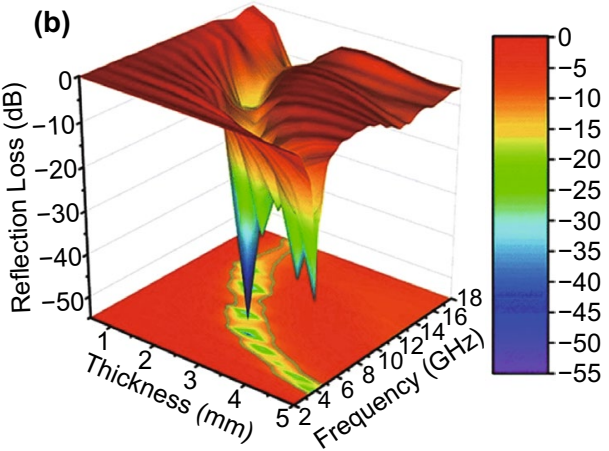

(f)

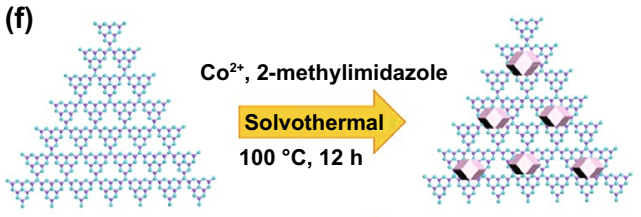

g- $\mathrm{C}_{3} \mathrm{~N}_{4}$

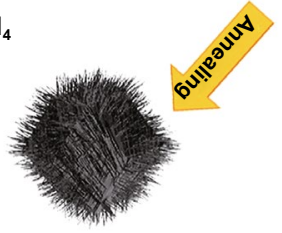

ZIF-67/g-C $\mathrm{C}_{3}$
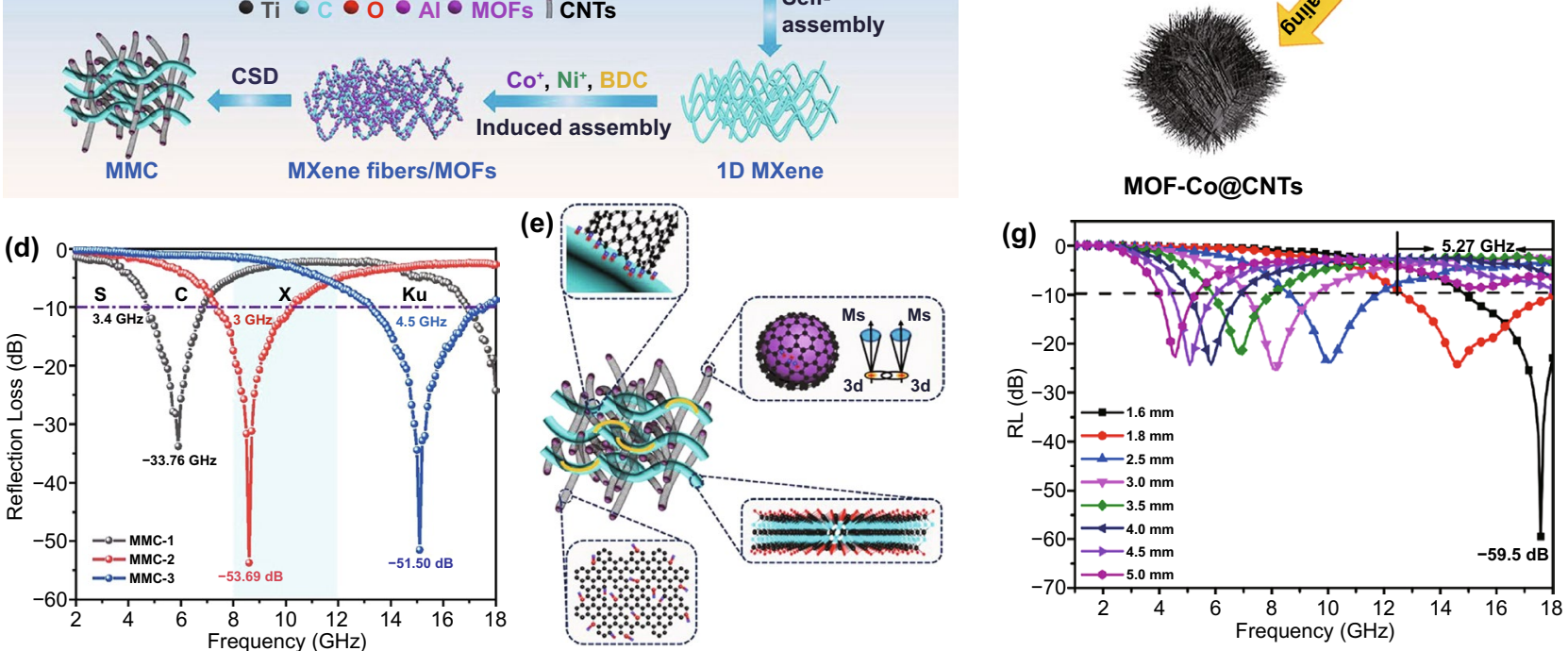

Fig. 16 a Schematic illustration of the synthesis and b 3D RL-f curves of $\mathrm{Fe}_{\mathrm{T}} \mathrm{TiO}_{2} @ \mathrm{C}$. Reprinted with permission from Ref. [156]. c Schematic description for preparation d RL-f curves and e MA mechanism diagram of MXene fibers@MOF-derived CNTs nanocomposites. Reprinted with permission from Ref. [59] f Diagrammatic sketch of the synthesis route and $\mathbf{g}$ RL-f curves of $\mathrm{ZIF}-67 / \mathrm{g}-\mathrm{C}_{3} \mathrm{~N}_{4}$. Reprinted with permission from Ref. [157]

properties. Many researchers have attempted to load MOFs onto biomaterials to serve as the precursors of MAMs, and this approach has been proved promising. One of the most common biomaterials is biomass cotton; Zhao et al. selected cotton/ZIF-67 as precursor to produce porous and fibrous structure of carbon-cotton/Co@NPC composite [131]. Compared with the product calcined by ZIF-67 and cotton alone, the MA performance of the carbon-cotton/Co@ NPC was significantly improved, the optimal performance includes $\mathrm{RL}_{\text {min }}$ of $-51.2 \mathrm{~dB}$ and $\mathrm{EAB}$ of $4.4 \mathrm{GHz}$ at only $1.65 \mathrm{~mm}$. Besides, Yang and $c o$-workers fabricated hollow carbon fiber/Co@C/CNTs by pyrolysis ZIF-67/cotton fiber in $\mathrm{H}_{2} / \mathrm{Ar}$ (Fig. 15a-c) [132], in which the interwoven fibers and CNTs promoted the formation of excellent conductive network. The ultra-lightweight hollow carbon fiber/Co@C/CNTs 
showed excellent MA ability with a $\mathrm{RL}_{\min }$ of $-53.5 \mathrm{~dB}$ at $7.8 \mathrm{GHz}$ and an EAB of $8.02 \mathrm{GHz}$ (Fig. 15d).

Other natural biomaterials, such as wood [147], walnut shell [148], and spinach [149], are also attracted the attention of researchers due to particular structure. As depicted in Fig. 16e, the Ni-MOF was uniformly grown on the surface of loofah by a microwave method, and three kinds of $\mathrm{Ni} / \mathrm{NC} / \mathrm{C}-\mathrm{T}$ ( $\mathrm{T}$ referred to pyrolysis temperatures) with different shapes were fabricated by carbonization (Fig. 15e) [146]. The Ni/NC/C-650 exhibited the best MA property $\left(\mathrm{RL}_{\min }=-63.1 \mathrm{~dB}, \mathrm{EAB}=5.12 \mathrm{GHz}\right)$, which was mainly due to the particular construction, high porosity, abundant interfaces, plentiful dipoles and the synergistic effect of magnetic loss and dielectric loss (Fig. 15f-g). Besides, Fei et al. designed ultra-lightweight aerogels of ZIF-67/bacterial cellulose (BC)-derived CNF@Co/C aerogels with high porosity and extremely low density, and the $\mathrm{CNF} @ \mathrm{Co} / \mathrm{C}$ aerogels displayed high EMI SE of $56.07 \mathrm{~dB}$ at ultra-low density [150].

\subsubsection{Loading on MXene}

As a novel class of two-dimensional material, metal carbides and nitrides (MXene) have attracted an increasing research attention in the field of microwave absorption due to their excellent metallic conductivity, unique layered structure, tunable active surface and outstanding electrical conductivity [151-154]. Han and co-workers designed MXene/CoZIF and MXene/Ni-ZIF precursors by coating Co-ZIF and $\mathrm{Ni}$-ZIF on the interlayer of MXene by the electrostatic selfassembly method [155]. The obtained two MAMs, labeled as MXene/Co-CZIF and MXene/Ni-CZIF, both achieved outstanding MA performance: the optimal $\mathrm{RL}_{\text {min }}$ of MXene/ Co-CZIF was $-60.09 \mathrm{~dB}$ at $7.36 \mathrm{GHz}$ with an ultrawide EAB of $9.3 \mathrm{GHz}$ and the measured $\mathrm{RL}_{\min }$ of MXene/Ni-CZIF was $-64.11 \mathrm{~dB}$ and the EAB was $4.56 \mathrm{GHz}$, in which the layered structure of MXene greatly enhanced the internal multiple dissipation of microwave to enhance the whole MA performance. Similarly, Deng et al. prepared $\mathrm{Fe} \& \mathrm{TiO}_{2} @ \mathrm{C}$ composites with MXene/Fe-MOFs as precursors by rapid microwave-assisted heating reaction and high-temperature carbonization [156]. The $\mathrm{Fe}$ and $\mathrm{TiO}_{2}$ NPs sandwiched between the MXene-derived carbon nanosheets to from a parallel multilayer structure, which greatly contributed to the attenuation of microwave and impedance matching of MA process (Fig. 16a). The $\mathrm{Fe} \& \mathrm{TiO}_{2} @ \mathrm{C}$ exhibited a $\mathrm{RL}_{\text {min }}$ of $-51.8 \mathrm{~dB}$ and a broad $\mathrm{EAB}$ of $6.5 \mathrm{GHz}$ with a low matching thickness of $1.6 \mathrm{~mm}$ (Fig. 16b).

Generally, the layer number of MXene can be regulated by adjusting the etching conditions. The single-layer MXene always exhibits higher electrical conductivity than multilayer MXene [152]. Wang et al. designed 3D hierarchical NiCo transition metal oxide@MXene composites by electrostatical self-assembly and high-temperature treatment, which exhibited great MA performance with $\mathrm{RL}_{\min }$ of $-67.22 \mathrm{~dB}$ at $1.70 \mathrm{~mm}$ and a broad $6.72 \mathrm{GHz}$ with a low filler loading of $5 \mathrm{wt} \%$. Wu synthesized novel MXene fibers@MOFderived CNTs nanocomposites (MMC) by annealing MXene fibers/CoNi-MOF (MM) precursors [59]. As indicated in Fig. 16c, the one-dimensional MXene fibers were obtained by self-assembly of two-dimensional MXene sheets under a strong alkaline environment. Then, the MM-x $(x=1,2,3$, representing the multiple of the added amount of $\mathrm{BDC}, \mathrm{Co}^{2+}$ and $\mathrm{Ni}^{2+}$ to the initial dose) was prepared by embedding CoNi-MOF on one-dimensional MXene fibers in suit and the MMC-x was obtained after carbonization. The MMCs exhibited excellent MA properties especially the $\mathrm{RL}_{\min }$ $(-51.6 \mathrm{~dB})$ of MMC-2 and EAB $(4.5 \mathrm{GHz})$ of MMC-3 (Fig. 16d). The MA mechanism is depicted in Fig. 16e, the layered porous structure promoted the multiple reflection of incident microwave and optimized impedance matching, the evenly distributed CoNi NPs provided the eddy current and natural resonance, and the defects, interfaces and interwoven $3 \mathrm{D}$ conductive networks equipped the MMC with excellent dielectric loss.

\subsubsection{Loading on $\mathrm{g}-\mathrm{C}_{3} \mathrm{~N}_{4}$}

Because of possessing abundant negative charge atoms, $\mathrm{N}$, graphitic carbon nitrides $\left(\mathrm{g}-\mathrm{C}_{3} \mathrm{~N}_{4}\right)$ display the strong ability to capture cations, which effectively promotes the combination with MOFs. However, the $\mathrm{g}_{-} \mathrm{C}_{3} \mathrm{~N}_{4}$, which is unstable at high temperatures, can be decomposed at $600{ }^{\circ} \mathrm{C}$ and almost transferred into cyanide fragments when the temperature is above $700{ }^{\circ} \mathrm{C}$. During the thermal decomposition process, the carbon materials are etched to increase porosity and the doped $\mathrm{N}$ can serve as polarization sites to enhance dipole polarization. Zhu synthesized MOF-Co@CNTs with ZIF$67 / g-\mathrm{C}_{3} \mathrm{~N}_{4}$ as precursors by the carbonization method [157]. The $\mathrm{g}_{-} \mathrm{C}_{3} \mathrm{~N}_{4}$ was prepared by pyrolyzing melamine, and the 

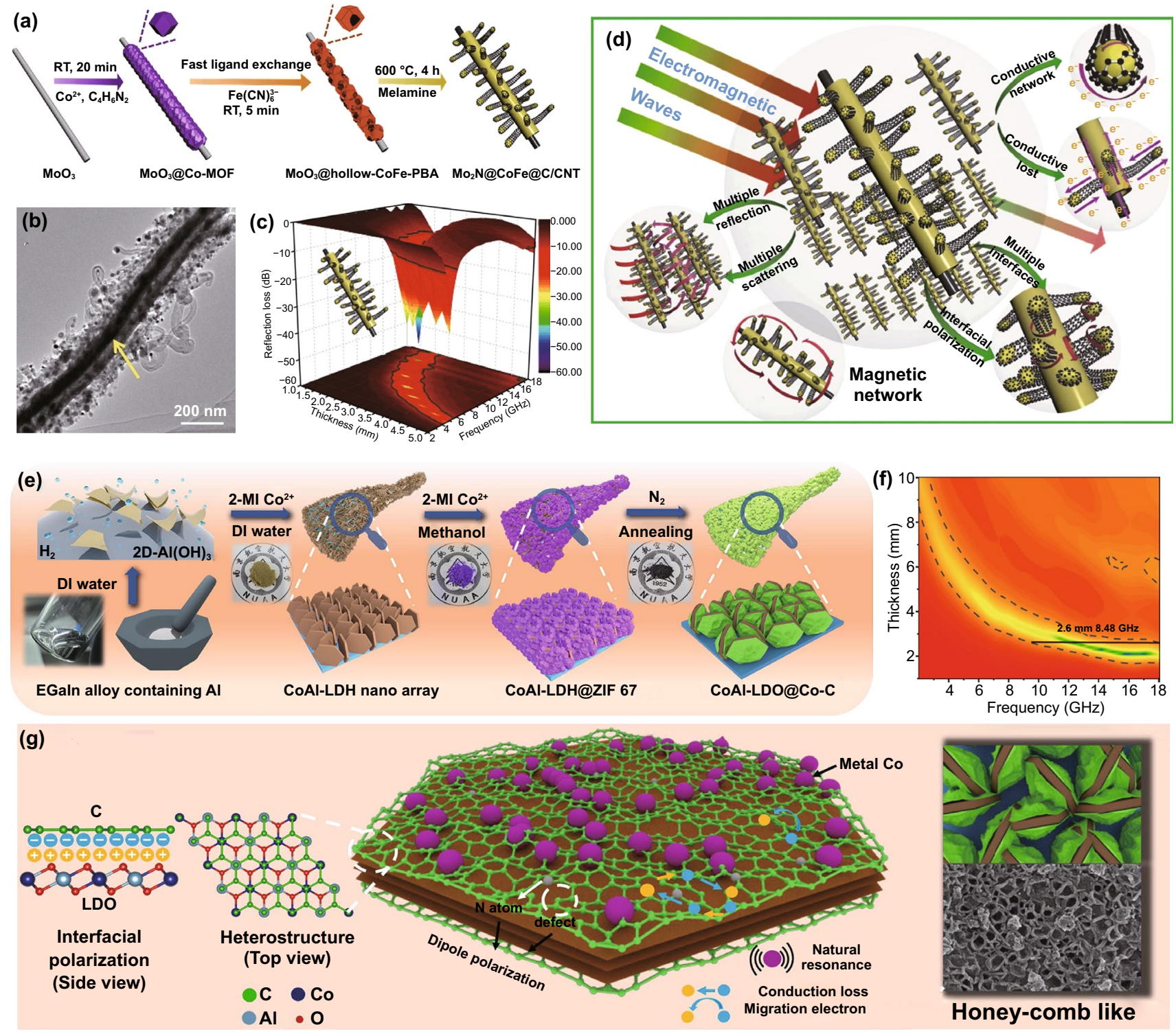

Fig. 17 a Schematic representation of the synthesis, b TEM image, c 3D RL-f curves and d MA mechanism of Mo ${ }_{2} \mathrm{~N} @ \mathrm{CoFe} @ \mathrm{C} / \mathrm{CNT}$. Reprinted with permission from Ref. [164]. e Schematic description for preparation route, $\mathbf{f} 2 \mathrm{D}$ RL-f mapping and $\mathbf{g}$ MA mechanism of CoAlLDO@Co-C. Reprinted with permission from Ref. [165]

ZIF-67 was uniformly embedded on $\mathrm{g}_{-} \mathrm{C}_{3} \mathrm{~N}_{4}$ (Fig. 16f). During the carbonization process, the organic skeleton and $\mathrm{Co}^{2+}$ of ZIF-67 were converted into graphitized carbon and Co NPs, respectively. Furthermore, the Co NPs could catalyze g- $\mathrm{C}_{3} \mathrm{~N}_{4}$ into CNTs growing on the surface of ZIF-67 to form conductive network, promoting electron transfer to enhance the conductivity loss. The MOF-Co@CNTs exhibited an excellent $\mathrm{RL}_{\text {min }}$ of $-59.5 \mathrm{~dB}$ with a layer thickness of only
$1.6 \mathrm{~mm}$ and a broad EAB of $5.27 \mathrm{GHz}$ at $1.8 \mathrm{~mm}$ (Fig. $16 \mathrm{~g}$ ). Besides, Jin and $c o$-workers used porous rod-shaped g- $\mathrm{C}_{3} \mathrm{~N}_{4}$ as template to grow ZIF-67 and then obtained micron-sized Co-doped porous carbon-based MAM $(\mathrm{CN} / \mathrm{CoC})$ at high temperature [158]. The $\mathrm{RL}_{\text {min }}$ and $\mathrm{EAB}$ of $\mathrm{CN} / \mathrm{CoC}$ were $-37.21 \mathrm{~dB}$ at $12.22 \mathrm{GHz}$ and $6.45 \mathrm{GHz}$. 


\subsubsection{Loading on Other Low-Dimensional Materials}

Conductive polymers, including polypyrrole, polythiophene and polyaniline,also draw an increasing research attention in the field of microwave absorption due to their excellent electrical conductivity $[10,159,160]$. For example, Sun and $c o$-workers prepared a chain-like polypyrrole (PPy) aerogel ornamented with ZIF-67-derived $\mathrm{Co} / \mathrm{C}$ composites (Co/C@ PPy aerogel) using the self-assembled polymerization method [161]. The $\mathrm{Co} / \mathrm{C}$ nanoparticles was mixed with pyrrole monomer and aged in mixture solvent of deionized water and anhydrite, and then aerogels were obtained after dialysis. The $\mathrm{Co} / \mathrm{C}$ nanoparticles were uniformly dispersed on the PPy chains, which constructed excellent three-dimensional conductive network to provide conductive loss. The $\mathrm{Co} / \mathrm{C} @ \mathrm{PPy}$ aerogel exhibited a high $\mathrm{RL}_{\min }$ of $-44.76 \mathrm{~dB}$ at $2 \mathrm{~mm}$.

Some oxides, nitrides and sulfides of transition metals (such as $\mathrm{ZnO}$ and $\mathrm{Mo}_{2} \mathrm{~S}$ ) with outstanding dielectric performance are also stimulated the research interest $[162,163]$. Che's group produced core-shell $\mathrm{MoO}_{3} @$ hollow-CoFe-PBA through in situ self-assembly and ligand exchange reaction of ZIF-67, than obtained the final $\mathrm{Mo}_{2} \mathrm{~N} @ \mathrm{CoFe} @ \mathrm{C} / \mathrm{CNT}$ composites by calcining with melamine (Fig. 17a) [164]. The CoFe nanoparticles acted as the catalyst of graphite carbon and CNTs which derived from melamine to grow in situ on the surface of $\mathrm{Mo}_{2} \mathrm{~N}$, constructing a novel "tubes on rods" structure (Fig. 17b). Impressive MA capability was achieved which exhibited a $\mathrm{RL}_{\min }$ of $-53.5 \mathrm{~dB}$ with a bandwidth of $5.0 \mathrm{GHz}$ at a low thickness of $2 \mathrm{~mm}$ (Fig. 17c). The microwave dissipation capacity of this MAM included the following four ways: interface polarization of heterogeneous interfaces, efficient electron transfer paths of C/CNTs and $\mathrm{Mo}_{2} \mathrm{~N}$ nanorods, magnetic loss of $\mathrm{CoFe}$ nanoparticles and multiple reflection created by layered core-shell structure (Fig. 17d).

Layered double hydroxides (LDH), lamellar compounds constructed by positively charged lamellae and negatively charged anions, are considered as a kind of promising MAMs because of their abundant active sites and chemical stability $[165,166]$. Zhang and co-workers designed ingenious CoAl-LDH@ZIF-67 using liquid metal alloydriven unique $2 \mathrm{D}$ hydroxide as template, in which the regular Co in CoAl-LDH served as the nucleation and growth sites to assist ZIF-67 embedded on CoAl-LDH stably [165]. The CoAl-LDO@Co-C nano-arrays were synthesized

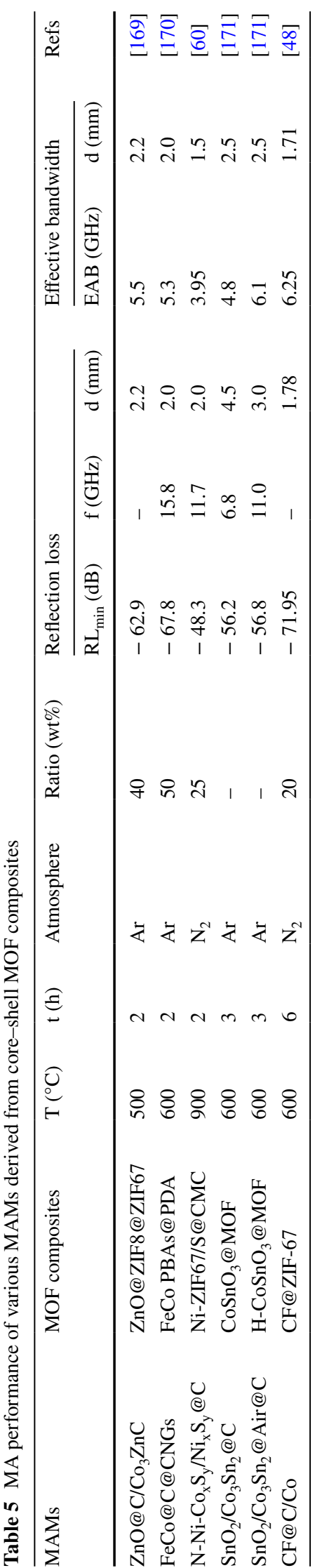


(a)

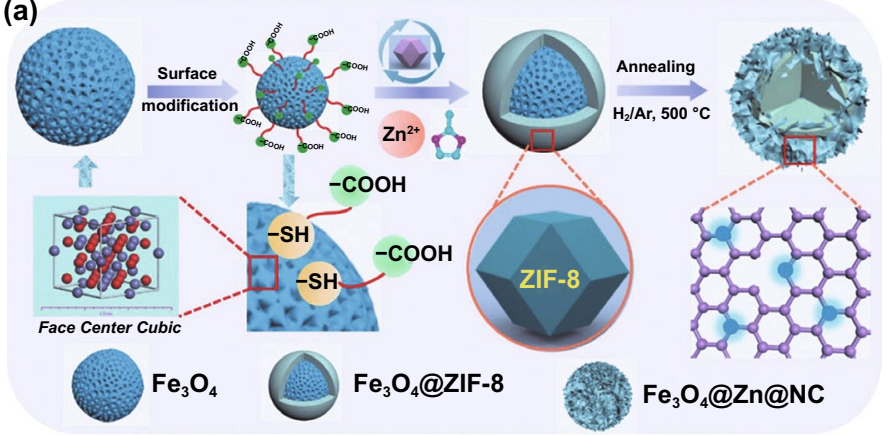

(b)

(c) 18
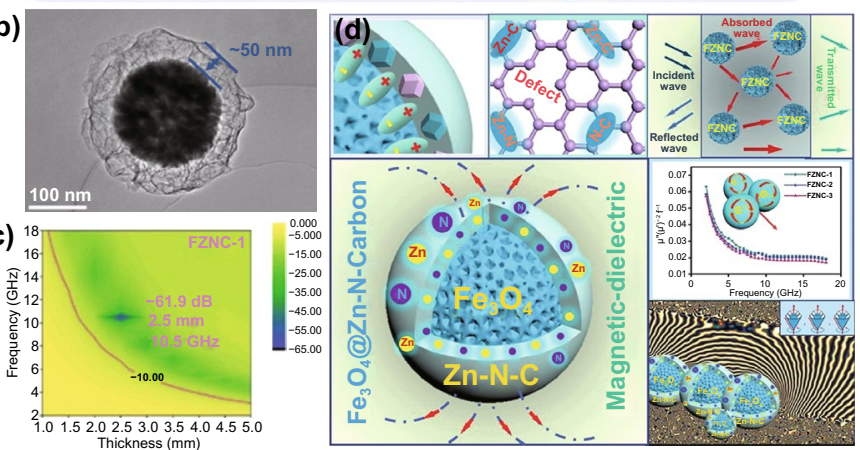

(e)

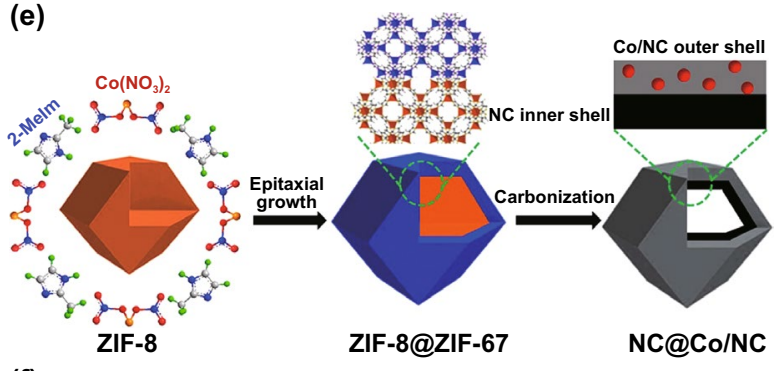

(f)
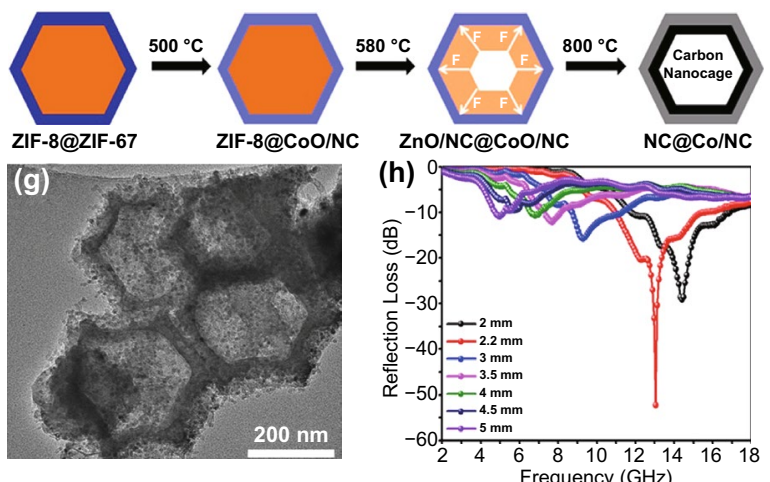

(i)
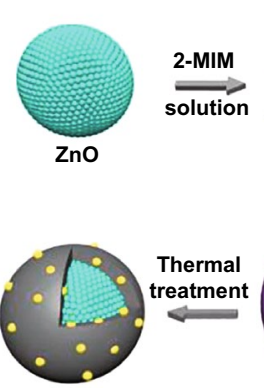
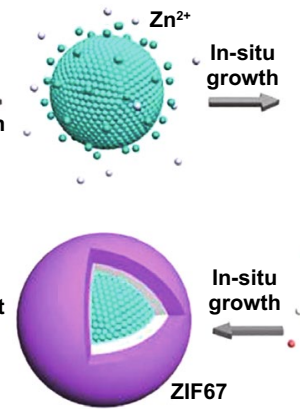
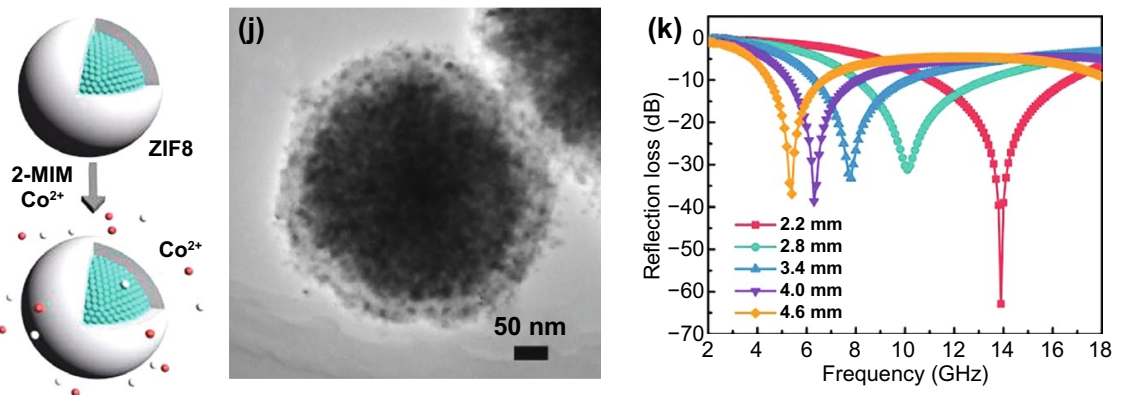

Fig. 18 a Diagrammatic sketch of the synthesis, b TEM image, $\mathbf{c} 2 \mathrm{D}$ RL-f mapping and $\mathbf{d}$ schematic diagram of MA mechanism of Fe $\mathrm{O}_{4} @$ $\mathrm{Zn}-\mathrm{N}$-Carbon. Reprinted with permission from Ref. [168]. e Schematic illustration of formation process, $\mathbf{f}$ synthetic mechanism, $\mathbf{g}$ TEM image and $\mathbf{h}$ RL-curves of NC@Co/NC carbon nanocages. Reprinted with permission from Ref. [172]. i Schematic illustration of the synthesis, $\mathbf{j}$ TEM image and $\mathbf{k}$ RL-curves of $\mathrm{ZnO} @$ carbon/ $\mathrm{Co}_{3} \mathrm{ZnC}$. Reprinted with permission from Ref. [169]

successfully after high-temperature treatment (Fig. 17e). The CoAl-LDO@Co-C achieved a relatively broad EAB of $8.48 \mathrm{GHz}$ at a thickness of $2.6 \mathrm{~mm}$, which surpassed a large amount of MOF-derived MAMs (Fig. 17f). The MA mechanism is shown in Fig. 17g, abundant heterojunction interfaces, defects and $\mathrm{N}$ atoms caused efficient polarization loss, graphitized carbon and uniformly dispersed Co magnetic loss provided great conduction loss and magnetic loss, and the honey-comb like structure supplied a favorable condition for the multiply reflection.

Some fibrous or rod-like materials with special properties are also studied as templates to load MOF precursors.
For instance, silicon carbide nanowires ( $\mathrm{SiC}$ NWs) with lightweight, large specific surface area and high stability were used as templates to grow ZIF-67 [136]. The pyrolysis product $\mathrm{Co} @ \mathrm{C} / \mathrm{SiC}$ displayed a $\mathrm{RL}_{\min }$ of $-47 \mathrm{~dB}$ and a EAB of $5.92 \mathrm{GHz}$, which mainly attributed to the multiple reflection loss derived from the great connectivity of $\mathrm{SiC}$ NWs and reasonable synergy of dielectric loss and magnetic loss. 


\subsection{Constructing Core-shell Structure}

Core-shell structure can assimilate the divided chemical and physical properties from both core and shell, easily overcoming the limitation of single component through various combinations of conductive materials and magnetic materials [167]. Table 5 lists the typical MAMs derived from core-shell MOF composites and their MA properties. For instance, Huang prepared a novel $\mathrm{Fe}_{3} \mathrm{O}_{4} @ \mathrm{Zn}-\mathrm{N}$-Carbon (FZNC) nanocomposite as a magnetic-dielectric synergy MAM [168]. As shown in Fig. $18 \mathrm{a}-\mathrm{b}, \mathrm{Fe}_{3} \mathrm{O}_{4}$ microsphere was modified with carboxyl group to provide deposition sites for $\mathrm{Zn}^{2+}$, and $\mathrm{ZIF}-8$ was further generated in situ to synthesis core-shell $\mathrm{Fe}_{3} \mathrm{O}_{4} @ \mathrm{ZIF}-8$. Then, the ZIF-8 shell transformed into $\mathrm{Zn}-\mathrm{N}$-Carbon layer coating the $\mathrm{Fe}_{3} \mathrm{O}_{4}$ magnetic core to obtain core-shell FZNC nanocomposite during the pyrolysis process. The FZNC achieved an optimized MA performance with a $\mathrm{RL}_{\min }$ of $-61.9 \mathrm{~dB}$ at $13.1 \mathrm{GHz}$ and a extremely wide EAB of $11.5 \mathrm{GHz}$ (Fig. 18c). The special structure constructed rich interfaces, polarization groups and defects, the graphitized carbon provided a pathway for electron migration, and the ingenious $\mathrm{FeO}-\mathrm{Fe}_{3} \mathrm{O}_{4}$ heterojunction interface improved the capacity of trapping electron/hole carriers and constructed the interfacial energy barrier (Fig. 18d).

It should be noted that the ZIF-67 could nucleate and grow on the ZIF- 8 because of their same crystal structures. In 2020, Liu designed a extraordinary $\mathrm{N}$-doped carbon nanocage with $\mathrm{NC}$ as inner shell and $\mathrm{Co} / \mathrm{NC}$ as outer shell (NC@ Co/NC) using the core-shell ZIF-8@ZIF-67 as precursor (Fig. 18e) [172]. The difference in thermal stability between ZIF-8 and ZIF-67 was the key to the successful preparation of NC@Co/NC carbon nanocage. In the carbonization process, ZIF-67 decomposed firstly to form the stable $\mathrm{Co} / \mathrm{NC}$ shell, which produced an outward adhesion force to inhibit the inward contraction of $\mathrm{NC}$ derived from ZIF-8 (Fig. 18f-g). The micro-meso-macropores improved impedance matching and reflection loss of NC@Co/NC, which exhibited outstanding MA property with a $\mathrm{RL}_{\min }$ of $-52.5 \mathrm{~dB}$ at $13.1 \mathrm{GHz}$ and EAB of $4.4 \mathrm{GHz}$ (Fig. 18h). Feng synthesized carbon-coated $\mathrm{ZnO}$ with $\mathrm{Co}_{3} \mathrm{ZnC}$ nanoparticles ( $\mathrm{ZnO} @$ carbon/ $\mathrm{Co}_{3} \mathrm{ZnC}$ ) embedded in the carbon matrix for microwave absorption [169]. The $\mathrm{ZnO}$ was used as template, and the ZIF-8 in the middle layer and ZIF-67 in the outermost layer grew on $\mathrm{ZnO}$; then, the $\mathrm{ZnO} @$ carbon/ $\mathrm{Co}_{3} \mathrm{ZnC}$ nanocomposites were successfully synthesized after the high-temperature treatment (Fig. $18 \mathrm{i}-\mathrm{j})$. The $\mathrm{RL}_{\min }$ of
$\mathrm{ZnO} @$ carbon $/ \mathrm{Co}_{3} \mathrm{ZnC}$ nanocomposites was dramatically enhanced to $-62.9 \mathrm{~dB}$ at $7.8 \mathrm{GHz}$, and the $\mathrm{EAB}$ was up to $5.5 \mathrm{GHz}$ (Fig. 18k).

\section{Summary and Outlook}

Recently, MOF-derived MAMs have made a great progress in microwave absorption field, acting as promising candidates for high-performance MAMs owing to their superior properties. In this review, various MOF-derived MAMs are systematically introduced and discussed. The MOF precursors can be classified into two major categories: pure MOFs and MOF composites. Numerous MOF-derived MAMs exhibited high MA performance with the $\mathrm{RL}_{\min }$ less than $-50 \mathrm{~dB}$ and the $\mathrm{EAB}$ greater than $4 \mathrm{GHz}$, proving huge application potential value of MOF-derived MAMs in the field of microwave absorption. In brief, the MA properties of MAMs mainly depend on the impedance matching and attenuation constant, which both relate to the capacity of dielectric loss and magnetic loss; we detailedly analyze the adjustment methods of the electromagnetic parameters from the aspects of component and structure design and summarize some promising methods to improve the MA capacity of MAMs. For MOF-derived MAMs, heat treatment condition is one of the most important factors to improve microwave attenuation performance; this paper analyzes the influence of pyrolysis temperature and atmosphere in the process of heat treatment and provides the reference for the future experimental parameter design.

There are some creative methods for constructing highperformance MAMs. (i) Enhancing the shape anisotropy. Generally, the magnetic metal could maintain a high permeability in the frequency range of $\mathrm{GHz}$ if the Snoek's limit is broke, and the introduction of shape anisotropy is considered as a promising method to realize this purpose $[67,173]$. Enhancing shape anisotropy is beneficial for the improvement of natural resonance frequency and coercivity [174]. Therefore, the anisotropic magnetic materials such as thin film, flat plate and wire are worth trying to construct MAMs with high performance. For instant, Liu and co-workers synthesized porous $\mathrm{Co} / \mathrm{Ni} / \mathrm{C}$ nanosheets with CoNi-MOF-71 as precursors [76]. The $\mathrm{Co} /$ $\mathrm{Ni} / \mathrm{C}$ nanosheets exhibited enhanced magnetic loss due to the great shape anisotropy of $2 \mathrm{D} \mathrm{Co} / \mathrm{Ni}$, which provided higher coercivity and natural resonance frequency because of the break of Snoek's limit. (ii) Widening aperture 
distribution. According to waveguide theory, the microwave could be dissipated inside the waveguide when the wavelength is twice the length of waveguide's cross section [175]. Furthermore, different pore sizes correspond to different microwave length, which indicates that the gradient distribution of pore structure is beneficial to broaden the EAB [149]. Besides, macropores can facilitate the impedance match of MAMs, while mesopores and micropores can enhance the multiple reflection of microwave inside the MAMs [109]. There are many approaches to construct MAMs with multiple pore sizes such as etching [176] and template method [42]. (iii) Constructing Schottky contact structure. When the metals contact with the semiconductors, the semiconductor band at the interfaces will be bent to construct Schottky barrier, forming a significant region of space charge. The presence of Schottky barrier could hinder the smooth electron transfer, resulting in the accumulation of electrons at the interface and the enhancement of interface polarization [174]. Gao et al. prepared unique Fe-ZnO Schottky contact polycrystals by ligand exchange strategy and carbonization method [174]. The results shown that appropriate Schottky junction can improve impedance mismatch and enhance microwave loss of MAMs. (iv) Introducing sulfur in the pyrolysis process. Compared with metal oxides, corresponding metal sulfides have a greater variety of valence states and structures, as well as better electrical conductivity [177]. The sulfur powder sublimates at $450{ }^{\circ} \mathrm{C}$, and the sulfur vapor can react with the metal atoms in the material to form sulfides with different valence states. $\mathrm{Wu}$ ' group prepared $\mathrm{C} / \mathrm{Cu} / \mathrm{Cu}_{2} \mathrm{O} @ \mathrm{Cu}_{2-\mathrm{x}} \mathrm{S}$ and $\mathrm{C} / \mathrm{Cu}_{2-\mathrm{x}} \mathrm{S} / \mathrm{CuS}$ absorbers using $\mathrm{Cu}-\mathrm{MOF}-74$ as precursors, indicating that the introduction of sulfide sulfur increased the number of dipoles and improve the conduction loss [178].

Although some achievements have been realized in the research and development of MOF-derived MAMs, there are still many significant challenges at this stage. First, the influence of various synthetic parameters on the synthesis of MOF-derived MAMs needs to be further explored. During preparation of MOFs, the reaction temperature and dosage ratio of raw materials, all have great effects on the sizes and morphologies of MOF precursors, which are directly related to the MA performance. Second, the microwave absorption mechanism needs to be further explored deeply. Microwave absorption capacity comes from the synergy of multi-mechanisms including dielectric loss, magnetic loss, multiple reflection and impedance matching, while the quantitative estimates on the influence of each mechanism still lack in sufficient theoretical supports. Besides, more well-designed MOF composites need to be made an attempt and deeply explored. The synergistic effect of multiple components is beneficial to the connectivity and energy conversion of electrical/magnetic networks in materials; MAMs derived from MOF composites are expected to bring new breakthroughs in this direction. Furthermore, MAMs with various excellent properties are waiting to be developed. In addition to strong absorption and wide band, the recently demand for MAMs is gradually developing toward the direction of high stability, multi-function and low cost to meet the requirements of actual application scenarios. Last, there is still a long way from research and development to industrial production and practical application. Current studies of MOF-derived MAMs only exist in the laboratory stage, and the mass production has not yet been achieved. In a word, the research on MOF-derived MAMs is still in the primary stage. The researchers still need to further clarify the MA mechanism and enrich the properties gradually based on a large number of experiments. In the meantime, researchers can scale up the experiment on the basis of the laboratory scale test and modify the process parameters during the pilot scale test, preparing for industrial production.

In conclusion, MOF-derived MAMs inject new blood into the field of microwave absorption due to their special structure, adjustable components and good electromagnetic properties. However, the research of MOF-derived MAMs is still in the infancy and there is much room for further research on the novel MOF-derived MAMs and their MA performance, in which the problems of practical application need to be focused on. We hope this comprehensive review can provide guidelines for the subsequent generation of MOFderived MAMs and firmly believe that the challenges and issues will be solved unceasingly and MOF-derived MAMs has prodigious potential to achieve new breakthroughs in microwave absorption.

Funding Open access funding provided by Shanghai Jiao Tong University.

Open Access This article is licensed under a Creative Commons Attribution 4.0 International License, which permits use, sharing, adaptation, distribution and reproduction in any medium or format, as long as you give appropriate credit to the original author(s) and the source, provide a link to the Creative Commons licence, and indicate if changes were made. The images or other third party material in this article are included in the article's Creative 
Commons licence, unless indicated otherwise in a credit line to the material. If material is not included in the article's Creative Commons licence and your intended use is not permitted by statutory regulation or exceeds the permitted use, you will need to obtain permission directly from the copyright holder. To view a copy of this licence, visit http://creativecommons.org/licenses/by/4.0/.

\section{References}

1. I. Yu, J. Ko, T.W. Kim, D.S. Lee, N.D. Kim et al., Effect of sorted, homogeneous electronic grade single-walled carbon nanotube on the electromagnetic shielding effectiveness. Carbon 167, 523-529 (2020). https://doi.org/10.1016/j.carbon. 2020.06.047

2. T. Lin, H. Yu, L. Wang, S. Fahad, A. Khan et al., A review of recent advances in the preparation of polyaniline-based composites and their electromagnetic absorption properties. J. Mater. Sci. 56, 5449-5478 (2021). https://doi.org/10.1007/ s10853-020-05631-1

3. H. Zhang, Z. Heng, J. Zhou, Y. Shi, Y. Chen et al., In-situ cocontinuous conductive network induced by carbon nanotubes in epoxy composites with enhanced electromagnetic interference shielding performance. Chem. Eng. J. 398, 125559 (2020). https://doi.org/10.1016/j.cej.2020.125559

4. Y. Bai, F. Qin, Y. Lu, Multifunctional electromagnetic interference shielding ternary alloy (Ni-W-P) decorated fabric with wide-operating-range joule heating performances. ACS Appl. Mater. Interfaces 12, 48016-48026 (2020). https://doi. org/10.1021/acsami.0c15134

5. A. Nazir, H. Yu, L. Wang, S. Fahad, K.-U.-R. Naveed et al., Electrical conductivity and electromagnetic interference shielding properties of polymer/carbon composites. J. Mater. Sci. Mater. 30, 16636-16650 (2019). https://doi.org/10.1007/ s10854-019-02043-z

6. R. Cheng, Y. Wang, X. Di, Z. Lu, P. Wang et al., Construction of MOF-derived plum-like NiCo@C composite with enhanced multi-polarization for high-efficiency microwave absorption. J. Colloid Interface Sci. 609, 224-234 (2022). https://doi.org/10.1016/j.jcis.2021.11.197

7. H. Geng, X. Zhang, W. Xie, P. Zhao, G. Wang et al., Lightweight and broadband 2D $\mathrm{MoS}_{2}$ nanosheets/3D carbon nanofibers hybrid aerogel for high-efficiency microwave absorption. J. Colloid Interface Sci. 609, 33-42 (2022). https://doi.org/10.1016/j.jcis.2021.11.192

8. Z. Zeng, C. Wang, G. Siqueira, D. Han, A. Huch et al., Nanocellulose-MXene biomimetic aerogels with orientation-tunable electromagnetic interference shielding performance. Adv. Sci. 7, 2000979 (2020). https://doi.org/10.1002/advs.20200 0979

9. H. Xu, X. Yin, X. Li, M. Li, S. Liang et al., Lightweight $\mathrm{Ti}_{2} \mathrm{CT}_{\mathrm{x}} \mathrm{MXene/Poly}$ (vinylalcohol) composite foams for electromagnetic wave shielding with absorption-dominated feature. ACS Appl. Mater. Interfaces 11, 10198-10207 (2019). https://doi.org/10.1021/acsami.8b21671
10. Y. Zhang, Z. Yang, T. Pan, H. Gao, H. Guan et al., Construction of natural fiber/polyaniline core-shell heterostructures with tunable and excellent electromagnetic shielding capability via a facile secondary doping strategy. Compos. Part A Appl. Sci. Manuf. 137, 105994 (2020). https://doi.org/10. 1016/j.compositesa.2020.105994

11. Y. Shi, L. He, D. Chen, Q. Wang, J. Shen et al., Simultaneously improved electromagnetic interference shielding and flame retarding properties of poly (butylene succinate)/thermoplastic polyurethane blends by constructing segregated flame retardants and multi-walled carbon nanotubes double network. Compos. Part A Appl. Sci. Manuf. 137, 106037 (2020). https://doi.org/10.1016/j.compositesa.2020.106037

12. T. Lin, H. Yu, Y. Wang, L. Wang, S.Z. Vatsadze et al., Polypyrrole nanotube/ferrocene-modified graphene oxide composites: from fabrication to EMI shielding application. J. Mater. Sci. 56, 18093-18115 (2021). https://doi.org/10. 1007/s10853-021-06406-y

13. T. Lin, H. Yu, L. Wang, Q. Ma, H. Huang et al., A study on the fabrication and microwave shielding properties of PANI/ $\mathrm{C}_{60}$ heterostructures. Polym. Compos. 42, 1961-1976 (2021). https://doi.org/10.1002/pc.25948

14. C. Chang, X. Yue, B. Hao, D. Xing, P. Ma, Direct growth of carbon nanotubes on basalt fiber for the application of electromagnetic interference shielding. Carbon 167, 31-39 (2020). https://doi.org/10.1016/j.carbon.2020.05.074

15. Y. Huang, K. Yasuda, C. Wan, Intercalation: constructing nanolaminated reduced graphene oxide/silica ceramics for lightweight and mechanically reliable electromagnetic interference shielding applications. ACS Appl. Mater. Interfaces 12, 55148-55156 (2020). https://doi.org/10.1021/acsami. $0 \mathrm{c} 15193$

16. A. Nazir, H. Yu, L. Wang, Y. He, Q. Chen et al., Electromagnetic interference shielding properties of ferrocene-based polypyrrole/carbon material composites. Appl. Phys. A-Mater. (2020). https://doi.org/10.1007/ s00339-020-03927-2

17. Y.B. Feng, T. Qiu, C.Y. Shen, Absorbing properties and structural design of microwave absorbers based on carbonyl iron and barium ferrite. J. Magn. Magn. Mater. 318, 8-13 (2007). https://doi.org/10.1016/j.jmmm.2007.04.012

18. G. Sun, B. Dong, M. Cao, B. Wei, C. Hu, Hierarchical dendrite-like magnetic materials of $\mathrm{Fe}_{3} \mathrm{O}_{4}, \gamma-\mathrm{Fe}_{2} \mathrm{O}_{3}$, and $\mathrm{Fe}$ with high performance of microwave absorption. Chem. Mater. 23, 1587-1593 (2011). https://doi.org/10.1021/cm103441u

19. Z. Wu, X. Qian, K. Pei, W. You, X. Li et al., Drawing advanced electromagnetic functional composites with ultralow filler loading. Chem. Eng. J. 399, 125720 (2020). https:// doi.org/10.1016/j.cej.2020.125720

20. X. Li, G. Ji, H. Lv, M. Wang, Y. Du, Microwave absorbing properties and enhanced infrared reflectance of $\mathrm{Fe} / \mathrm{Cu}$ composites prepared by chemical plating. J. Magn. Magn. Mater. 355, 65-69 (2014). https://doi.org/10.1016/j.jmmm.2013.11. 055

21. H. Lv, G. Ji, X. Li, X. Chang, M. Wang et al., Microwave absorbing properties and enhanced infrared reflectance of 
FeAl mixture synthesized by two-step ball-milling method. J. Magn. Magn. Mater. 374, 225-229 (2015). https://doi.org/ 10.1016/j.jmmm.2014.08.006

22. A.J. Albaaji, E.G. Castle, M.J. Reece, J.P. Hall, S.L. Evans, Effect of ball-milling time on mechanical and magnetic properties of carbon nanotube reinforced FeCo alloy composites. Mater. Des. 122, 296-306 (2017). https://doi.org/10.1016/j. matdes.2017.02.091

23. R. Guil-Lopez, R.M. Navarro, J.L.G. Fierro, Controlling the impregnation of nickel on nanoporous aluminum oxide nanoliths as catalysts for partial oxidation of methane. Chem. Eng. J. 256, 458-467 (2014). https://doi.org/10.1016/j.cej.2014.05. 146

24. Z. Huang, H. Yu, L. Wang, X. Liu, T. Lin et al., Ferrocenecontained metal organic frameworks: from synthesis to applications. Coord. Chem. Rev. 430, 213737 (2021). https://doi. org/10.1016/j.ccr.2020.213737

25. J. Liu, Z. Deng, H. Yu, L. Wang, Ferrocene-based metalorganic framework for highly efficient recovery of gold from WEEE. Chem. Eng. J. 410, 128360 (2021). https://doi.org/ 10.1016/j.cej.2020.128360

26. Z. Deng, H. Yu, L. Wang, J. Liu, K.J. Shea, Ferrocene-based metal-organic framework nanosheets loaded with palladium as a super-high active hydrogenation catalyst. J. Mater. Chem. A 7, 15975-15980 (2019). https://doi.org/10.1039/c9ta0 $3403 \mathrm{j}$

27. J. Liu, L. Chen, H. Cui, J. Zhang, L. Zhang et al., Applications of metal-organic frameworks in heterogeneous supramolecular catalysis. Chem. Soc. Rev. 43, 6011-6061 (2014). https://doi.org/10.1039/c4cs00094c

28. W. Xia, A. Mahmood, R. Zou, Q. Xu, Metal-organic frameworks and their derived nanostructures for electrochemical energy storage and conversion. Energy Environ. Sci. 8, 18371866 (2015). https://doi.org/10.1039/C5EE00762C

29. X. Cao, C. Tan, M. Sindoro, H. Zhang, Hybrid micro- nanostructures derived from metal-organic frameworks: preparation and applications in energy storage and conversion. Chem. Soc. Rev. 46, 2660-2677 (2017). https://doi.org/10. 1039/C6CS00426a

30. T. Rodenas, I. Luz, G. Prieto, B. Seoane, H. Miro et al., Metal-organic framework nanosheets in polymer composite materials for gas separation. Nat. Mater. 14, 48-55 (2015). https://doi.org/10.1038/nmat4113

31. J.-R. Li, R.J. Kuppler, H.-C. Zhou, Selective gas adsorption and separation in metal-organic frameworks. Chem. Soc. Rev. 38, 1477-1504 (2009). https://doi.org/10.1039/b802426j

32. W.P. Lustig, S. Mukherjee, N.D. Rudd, A.V. Desai, J. Li et al., Metal-organic frameworks: functional luminescent and photonic materials for sensing applications. Chem. Soc. Rev. 46, 3242-3285 (2017). https://doi.org/10.1039/C6CS00930A

33. Z. Hu, B.J. Deibert, J. Li, Luminescent metal-organic frameworks for chemical sensing and explosive detection. Chem. Soc. Rev. 43, 5815-5840 (2014). https://doi.org/10.1039/ $\mathrm{c} 4 \mathrm{cs} 00010 \mathrm{~b}$

34. R. Jin, C. Zeng, M. Zhou, Y. Chen, Atomically precise colloidal metal nanoclusters and nanoparticles: fundamentals and opportunities. Chem. Rev. 116, 10346-10413 (2016). https:// doi.org/10.1021/acs.chemrev.5b00703

35. Y. Lu, Y. Wang, H. Li, Y. Lin, Z. Jiang et al., MOF-derived porous $\mathrm{Co} / \mathrm{C}$ nanocomposites with excellent electromagnetic wave absorption properties. ACS Appl. Mater. Interfaces 7, 13604-13611 (2015). https://doi.org/10.1021/acsami.5b031 77

36. J. Yan, Y. Huang, X. Han, X. Gao, P. Liu, Metal organic framework (ZIF-67)-derived hollow $\mathrm{CoS}_{2} / \mathrm{N}$-doped carbon nanotube composites for extraordinary electromagnetic wave absorption. Compos. B. Eng. 163, 67-76 (2019). https://doi. org/10.1016/j.compositesb.2018.11.008

37. L. Wang, X. Bai, B. Wen, Z. Du, Y. Lin, Honeycomb-like $\mathrm{Co} / \mathrm{C}$ composites derived from hierarchically nanoporous ZIF-67 as a lightweight and highly efficient microwave absorber. Compos. B Eng. 166, 464-471 (2019). https://doi. org/10.1016/j.compositesb.2019.02.054

38. W. Liu, Q. Shao, G. Ji, X. Liang, Y. Cheng et al., Metalorganic-frameworks derived porous carbon-wrapped $\mathrm{Ni}$ composites with optimized impedance matching as excellent lightweight electromagnetic wave absorber. Chem. Eng. J. 313, 734-744 (2017). https://doi.org/10.1016/j.cej.2016.12. 117

39. P. Miao, K. Cheng, H. Li, J. Gu, K. Chen et al., Poly(dimethylsilylene)diacetylene-guided ZIF-based heterostructures for full $\mathrm{Ku}$-band electromagnetic wave absorption. ACS Appl. Mater. Interfaces 11, 17706-17713 (2019). https://doi.org/10.1021/acsami.9b03944

40. L. Wang, X. Yu, X. Li, J. Zhang, M. Wang et al., MOFderived yolk-shell Ni@C@ZnO Schottky contact structure for enhanced microwave absorption. Chem. Eng. J. 383, 123099 (2020). https://doi.org/10.1016/j.cej.2019.123099

41. Y. Qiu, Y. Lin, H. Yang, L. Wang, M. Wang et al., Hollow $\mathrm{Ni} / \mathrm{C}$ microspheres derived from Ni-metal organic framework for electromagnetic wave absorption. Chem. Eng. J. 383, 123207 (2020). https://doi.org/10.1016/j.cej.2019.123207

42. Z. Li, X. Han, Y. Ma, D. Liu, Y. Wang et al., MOFs-derived hollow $\mathrm{Co} / \mathrm{C}$ microspheres with enhanced microwave absorption performance. ACS Sustain. Chem. Eng. 6, 8904-8913 (2018). https://doi.org/10.1021/acssuschemeng.8b01270

43. Z. Yang, H. Lv, R. Wu, Rational construction of graphene oxide with MOF-derived porous NiFe@C nanocubes for high-performance microwave attenuation. Nano Res. 9, 36713682 (2016). https://doi.org/10.1007/s12274-016-1238-Z

44. H. Fan, Z. Yao, J. Zhou, P. Yi, B. Wei et al., Enhanced microwave absorption of epoxy composite by constructing 3D Co-C-MWCNTs derived from metal organic frameworks. J. Mater. Sci. 56, 1426-1442 (2021). https://doi.org/10.1007/ s10853-020-05365-0

45. S. Song, A. Zhang, L. Chen, Q. Jia, C. Zhou et al., A novel multi-cavity structured MOF derivative-porous graphene hybrid for high performance microwave absorption. Carbon 176, 279-289 (2021). https://doi.org/10.1016/j.carbon.2021. 01.138

46. M. Kong, Z. Jia, B. Wang, J. Dou, X. Liu et al., Construction of metal-organic framework derived $\mathrm{Co} / \mathrm{ZnO} / \mathrm{Ti}_{3} \mathrm{C}_{2} \mathrm{~T}_{\mathrm{x}}$ 
composites for excellent microwave absorption. Sustain. Mater. Technol. 26, e00219 (2020). https://doi.org/10.1016/j. susmat.2020.e00219

47. W. Liu, S. Tan, Z. Yang, G. Ji, Enhanced low-frequency electromagnetic properties of MOF-derived cobalt through interface design. ACS Appl. Mater. Interfaces 10, 31610-31622 (2018). https://doi.org/10.1021/acsami.8b10685

48. J. Tao, Z. Jiao, L. Xu, P. Yi, Z. Yao et al., Construction of MOF-derived $\mathrm{Co} / \mathrm{C}$ shell on carbon fiber surface to enhance multi-polarization effect towards efficient broadband electromagnetic wave absorption. Carbon 184, 571-582 (2021). https://doi.org/10.1016/j.carbon.2021.08.064

49. X. Xu, F. Ran, Z. Fan, Z. Cheng, T. Lv et al., Bimetallic metal-organic framework-derived pomegranate-like nanoclusters coupled with $\mathrm{CoNi}$-doped graphene for strong wideband microwave absorption. ACS Appl. Mater. Interfaces 12, 17870-17880 (2020). https://doi.org/10.1021/acsami.0c015 72

50. Z. Zhang, Z. Cai, Z. Wang, Y. Peng, L. Xia et al., A review on metal-organic framework derived porous carbon-based novel microwave absorption materials. Nano-Micro Lett. 13, 56 (2021). https://doi.org/10.1007/s40820-020-00582-3

51. N. Wu, D. Xu, Z. Wang, F. Wang, J. Liu et al., Achieving superior electromagnetic wave absorbers through the novel metal-organic frameworks derived magnetic porous carbon nanorods. Carbon 145, 433-444 (2019). https://doi.org/10. 1016/j.carbon.2019.01.028

52. K. Sushmita, G. Madras, S. Bose, Polymer nanocomposites containing semiconductors as advanced materials for EMI shielding. ACS Omega 5, 4705-4718 (2020). https://doi.org/ 10.1021/acsomega.9b03641

53. D. Jiang, V. Murugadoss, Y. Wang, J. Lin, T. Ding et al., Electromagnetic interference shielding polymers and nanocomposites-a review. Polym. Rev. 59, 280-337 (2019). https://doi. org/10.1080/15583724.2018.1546737

54. Z. Jia, M. Zhang, B. Liu, F. Wang, G. Wei et al., Graphene foams for electromagnetic interference shielding: a review. ACS Appl. Nano Mater. 3, 6140-6155 (2020). https://doi. org/10.1021/acsanm.0c00835

55. R. Pastore, A. Delfini, D. Micheli, A. Vricella, M. Marchetti et al., Carbon foam electromagnetic mm-wave absorption in reverberation chamber. Carbon 144, 63-71 (2019). https:// doi.org/10.1016/j.carbon.2018.12.026

56. A. Iqbal, P. Sambyal, C.M. Koo, 2D MXenes for electromagnetic shielding: a review. Adv. Funct. Mater. 30, 2000883 (2020). https://doi.org/10.1002/adfm.202000883

57. X. Li, X. Yin, C. Song, M. Han, H. Xu et al., Self-assembly core-shell graphene-bridged hollow MXenes spheres 3D foam with ultrahigh specific EM absorption performance. Adv. Funct. Mater. 28, 1803938 (2018). https://doi.org/10. 1002/adfm.201803938

58. L. Li, S. Zhao, X. Luo, H. Zhang, Z. Yu, Smart MXene-based janus films with multi-responsive actuation capability and high electromagnetic interference shielding performances. Carbon 175, 594-602 (2021). https://doi.org/10.1016/j.carbon.2020.10.090
59. F. Wu, Z. Liu, J. Wang, T. Shah, P. Liu et al., Template-free self-assembly of MXene and CoNi-bimetal MOF into intertwined one-dimensional heterostructure and its microwave absorbing properties. Chem. Eng. J. 422, 130591 (2021). https://doi.org/10.1016/j.cej.2021.130591

60. G. Song, K. Yang, L. Gai, Y. Li, Q. An et al., ZIF-67-CMCderived 3D N-doped hierarchical porous carbon with in-situ encapsulated bimetallic sulfide and Ni NPs for synergistic microwave absorption. Compos. Part A Appl. Sci. Manuf. 149, 106584 (2021). https://doi.org/10.1016/j.compositesa. 2021.106584

61. Y. Zhou, S. Wang, D. Li, L. Jiang, Lightweight and recoverable ANF/rGO/PI composite aerogels for broad and highperformance microwave absorption. Compos. B Eng. 213, 108701 (2021). https://doi.org/10.1016/j.compositesb.2021. 108701

62. Z. Liu, F. Pan, B. Deng, Z. Xiang, W. Lu, Self-assembled $\mathrm{MoS}_{2}$-3D worm-like expanded graphite hybrids for highefficiency microwave absorption. Carbon 174, 59-69 (2021). https://doi.org/10.1016/j.carbon.2020.12.019

63. X. Xu, F. Ran, Z. Fan, H. Lai, Z. Cheng et al., Cactus-inspired bimetallic metal-organic framework-derived 1D-2D hierarchical $\mathrm{Co} / \mathrm{N}$-decorated carbon architecture toward enhanced electromagnetic wave absorbing performance. ACS Appl. Mater. Interfaces 11, 13564-13573 (2019). https://doi.org/ 10.1021/acsami.9b00356

64. J. Chen, J. Zheng, F. Wang, Q. Huang, G. Ji, Carbon fibers embedded with $\mathrm{Fe}^{\mathrm{III}}$-MOF-5-derived composites for enhanced microwave absorption. Carbon 174, 509-517 (2021). https://doi.org/10.1016/j.carbon.2020.12.077

65. X. Zhang, J. Xu, X. Liu, S. Zhang, H. Yuan et al., Metal organic framework-derived three-dimensional graphenesupported nitrogen-doped carbon nanotube spheres for electromagnetic wave absorption with ultralow filler mass loading. Carbon 155, 233-242 (2019). https://doi.org/10.1016/j. carbon.2019.08.074

66. L. Wang, M. Huang, X. Yu, W. You, J. Zhang et al., MOFderived $\mathrm{Ni}_{1-\mathrm{x}} \mathrm{Co}_{\mathrm{x}} @$ Carbon with tunable nano-microstructure as lightweight and highly efficient electromagnetic wave absorber. Nano-Micro Lett. 12, 150 (2020). https://doi.org/ 10.1007/s40820-020-00488-0

67. C. Wu, K. Bi, M. Yan, Scalable self-supported $\mathrm{FeNi}_{3} / \mathrm{Mo}_{2} \mathrm{C}$ flexible paper for enhanced electromagnetic wave absorption evaluated via coaxial, waveguide and arch methods. J. Mater. Chem. C 8, 10204-10212 (2020). https://doi.org/10.1039/ d0tc01881c

68. Y. Yin, X. Liu, X. Wei, Y. Li, X. Nie et al., Magnetically aligned Co-C/MWCNTs composite derived from MWCNTinterconnected zeolitic imidazolate frameworks for a lightweight and highly efficient electromagnetic wave absorber. ACS Appl. Mater. Interfaces 9, 30850-30861 (2017). https:// doi.org/10.1021/acsami.7b10067

69. J. Chen, J. Zheng, Q. Huang, G. Wang, G. Ji, Carbon fibers@ Co-ZIFs derivations composites as highly efficient electromagnetic wave absorbers. J. Mater. Sci. Technol. 94, 239-246 (2021). https://doi.org/10.1016/j.jmst.2021.03.072 
70. M. Kong, X. Liu, Z. Jia, B. Wang, X. Wu et al., Porous magnetic carbon CoFe alloys@ZnO@C composites based on $\mathrm{Zn} / \mathrm{Co}$-based bimetallic MOF with efficient electromagnetic wave absorption. J. Colloid Interface Sci. 604, 39-51 (2021). https://doi.org/10.1016/j.jcis.2021.07.003

71. B. Zhao, Y. Li, H. Ji, P. Bai, S. Wang et al., Lightweight graphene aerogels by decoration of 1D CoNi chains and CNTs to achieve ultra-wide microwave absorption. Carbon 176, 411-420 (2021). https://doi.org/10.1016/j.carbon.2021.01. 136

72. Y. Zhao, W. Wang, Q. Wang, H. Zhao, P. Li et al., Construction of excellent electromagnetic wave absorber from multiheterostructure materials derived from $\mathrm{ZnCo}_{2} \mathrm{O}_{4}$ and $\mathrm{ZIF}-67$ composite. Carbon 185, 514-525 (2021). https://doi.org/10. 1016/j.carbon.2021.09.049

73. W. Wang, H. Zhang, Y. Zhao, J. Wang, H. Zhao et al., A novel MOF-drived self-decomposition strategy for $\mathrm{CoO} @ \mathrm{~N} / \mathrm{C}-\mathrm{Co} /$ $\mathrm{Ni}-\mathrm{NiCo}_{2} \mathrm{O}_{4}$ multi-heterostructure composite as high-performance electromagnetic wave absorbing materials. Chem. Eng. J. 426, 131667 (2021). https://doi.org/10.1016/j.cej. 2021.131667

74. J. Wang, M. Zhou, Z. Xie, X. Hao, S. Tang et al., Enhanced interfacial polarization of biomass-derived porous carbon with a low radar cross-section. J. Colloid Interface Sci. 612, 146-155 (2021). https://doi.org/10.1016/j.jcis.2021.12.162

75. H. Lv, C. Wu, F. Qin, H. Peng, M. Yan, Extra-wide bandwidth via complementary exchange resonance and dielectric polarization of sandwiched FeNi@SnO nanosheets for electromagnetic wave absorption. J. Mater. Sci. Technol. 90, 1-8 (2021). https://doi.org/10.1016/j.jmst.2020.12.083

76. G. Liu, J. Tu, C. Wu, Y. Fu, C. Chu et al., High-yield twodimensional metal-organic framework derivatives for wideband electromagnetic wave absorption. ACS Appl. Mater. Interfaces 13, 20459-20466 (2021). https://doi.org/10.1021/ acsami. $1 \mathrm{c} 00281$

77. Y. Wang, H. Wang, J. Ye, L. Shi, X. Feng, Magnetic CoFe alloy@C nanocomposites derived from $\mathrm{ZnCo-MOF}$ for electromagnetic wave absorption. Chem. Eng. J. 383, 123096 (2020). https://doi.org/10.1016/j.cej.2019.123096

78. Q. Wang, J. Wang, Y. Zhao, Y. Zhao, J. Yan et al., NiO/ $\mathrm{NiFe}_{2} \mathrm{O}_{4} @ \mathrm{~N}$-doped reduced graphene oxide aerogel towards the wideband electromagnetic wave absorption: experimental and theoretical study. Chem. Eng. J. 430, 132814 (2022). https://doi.org/10.1016/j.cej.2021.132814

79. X. Yang, B. Fan, X. Tang, J. Wang, G. Tong et al., Interface modulation of chiral $\mathrm{PPy} / \mathrm{Fe}_{3} \mathrm{O}_{4}$ planar microhelixes to achieve electric/magnetic-coupling and wide-band microwave absorption. Chem. Eng. J. 430, 132747 (2022). https://doi. org/10.1016/j.cej.2021.132747

80. Y. Wang, W. Zhou, G. Zeng, H. Chen, H. Luo et al., Rational design of multi-shell hollow carbon submicrospheres for high-performance microwave absorbers. Carbon 175, 233242 (2021). https://doi.org/10.1016/j.carbon.2021.01.001

81. J. Wang, F. Wu, Y. Cui, A. Zhang, Q. Zhang et al., Efficient synthesis of $\mathrm{N}$-doped porous carbon nanoribbon composites with selective microwave absorption performance in common wavebands. Carbon 175, 164-175 (2021). https://doi.org/10. 1016/j.carbon.2021.01.005

82. S. Peng, S. Wang, G. Hao, C. Zhu, Y. Zhang et al., Preparation of magnetic flower-like carbon-matrix composites with efficient electromagnetic wave absorption properties by carbonization of MIL-101(Fe). J. Magn. Magn. Mater. 487, 165306 (2019). https://doi.org/10.1016/j.jmmm.2019.165306

83. Z. Xiang, Y. Song, J. Xiong, Z. Pan, X. Wang et al., Enhanced electromagnetic wave absorption of nanoporous $\mathrm{Fe}_{3} \mathrm{O}_{4} @$ carbon composites derived from metal-organic frameworks. Carbon 142, 20-31 (2019). https://doi.org/10.1016/j.carbon. 2018.10.014

84. K. Wang, Y. Chen, R. Tian, H. Li, Y. Zhou et al., Porous Co-C core-shell nanocomposites derived from Co-MOF-74 with enhanced electromagnetic wave absorption performance. ACS Appl. Mater. Interfaces 10, 11333-11342 (2018). https://doi.org/10.1021/acsami.8b00965

85. M. Huang, L. Wang, K. Pei, W. You, X. Yu et al., Multidimension-controllable synthesis of MOF-derived Co@Ndoped carbon composite with magnetic-dielectric synergy toward strong microwave absorption. Small 16, 2000158 (2020). https://doi.org/10.1002/smll.202000158

86. Q. Zeng, L. Wang, X. Li, W. You, J. Zhang et al., Double ligand MOF-derived pomegranate-like $\mathrm{Ni} @ \mathrm{C}$ microspheres as high-performance microwave absorber. Appl. Surf. Sci. 538, 148051 (2021). https://doi.org/10.1016/j.apsusc.2020. 148051

87. J. Yan, Y. Huang, Y. Yan, L. Ding, P. Liu, High-performance electromagnetic wave absorbers based on two kinds of nickelbased MOF-derived Ni@C microspheres. ACS Appl. Mater. Interfaces 11, 40781-40792 (2019). https://doi.org/10.1021/ acsami.9b12850

88. Z. Yang, Y. Zhang, M. Li, L. Yang, J. Liu et al., Surface architecture of Ni-based metal organic framework hollow spheres for adjustable microwave absorption. ACS Appl. Nano Mater. 2, 7888-7897 (2019). https://doi.org/10.1021/acsanm.9b018 81

89. S. Zhong, C. Zhan, D. Cao, Zeolitic imidazolate frameworkderived nitrogen-doped porous carbons as high performance supercapacitor electrode materials. Carbon 85, 51-59 (2015). https://doi.org/10.1016/j.carbon.2014.12.064

90. J. Ma, W. Liu, X. Liang, B. Quan, Y. Cheng et al., Nanoporous $\mathrm{TiO}_{2} / \mathrm{C}$ composites synthesized from directly pyrolysis of a Ti-based MOFs MIL-125(Ti) for efficient microwave absorption. J. Alloys Compd. 728, 138-144 (2017). https:// doi.org/10.1016/j.jallcom.2017.08.274

91. X. Zhang, J. Qiao, C. Liu, F. Wang, Y. Jiang et al., A MOFderived $\mathrm{ZrO}_{2} / \mathrm{C}$ nanocomposite for efficient electromagnetic wave absorption. Inorg. Chem. Front. 7, 385-393 (2020). https://doi.org/10.1039/c9qi01259a

92. R. Qiang, Y. Du, H. Zhao, Y. Wang, C. Tian et al., Metal organic framework-derived Fe-C nanocubes toward efficient microwave absorption. J. Mater. Chem. A 3, 13426-13434 (2015). https://doi.org/10.1039/C5TA01457C

93. P. Miao, R. Zhou, K. Chen, J. Liang, Q. Ban et al., Tunable electromagnetic wave absorption of supramolecular 
isomer-derived nanocomposites with different morphology. Adv. Mater. Interfaces 7, 1901820 (2020). https://doi.org/10. 1002/admi.201901820

94. P. Li, D.E. Miser, S. Rabiei, R.T. Yadav, M.R. Hajaligol, The removal of carbon monoxide by iron oxide nanoparticles. Appl. Catal. B 43, 151-162 (2003). https://doi.org/10.1016/ s0926-3373(02)00297-7

95. W. Liu, L. Liu, G. Ji, D. Li, Y. Zhang et al., Composition design and structural characterization of MOF-derived composites with controllable electromagnetic properties. ACS Sustain. Chem. Eng. 5, 7961-7971 (2017). https://doi.org/ 10.1021/acssuschemeng.7b01514

96. C. Peng, Y. Zhang, B. Zhang, MOF-derived jujube pit shaped $\mathrm{C} / \mathrm{Co}$ composites with hierarchical structure for electromagnetic absorption. J. Alloys Compd. 826, 154203 (2020). https://doi.org/10.1016/j.jallcom.2020.154203

97. Q. Wu, H. Jin, W. Chen, S. Huo, X. Chen et al., Graphitized nitrogen-doped porous carbon composites derived from ZIF-8 as efficient microwave absorption materials. Mater. Res. Express 5, 065602 (2018). https://doi.org/10.1088/20531591/aac67e

98. Y. Dong, X. Zhu, F. Pan, B. Deng, Z. Liu et al., Mace-like carbon fiber/ZnO nanorod composite derived from typha orientalis for lightweight and high-efficient electromagnetic wave absorber. Adv. Compos. Hybrid Ma. 4, 1002-1014 (2021). https://doi.org/10.1007/s42114-021-00277-2

99. J. Wang, B. Wang, Z. Wang, L. Chen, C. Gao et al., Synthesis of $3 \mathrm{D}$ flower-like $\mathrm{ZnO} / \mathrm{ZnCo}_{2} \mathrm{O}_{4}$ composites with the heterogeneous interface for excellent electromagnetic wave absorption properties. J. Colloid Interface Sci. 586, 479-490 (2021). https://doi.org/10.1016/j.jcis.2020.10.111

100. G. He, Y. Duan, H. Pang, J. Hu, Superior microwave absorption based on $\mathrm{ZnO}$ capped $\mathrm{MnO}_{2}$ nanostructures. Adv. Mater. Interfaces 7, 2000407 (2020). https://doi.org/10.1002/admi. 202000407

101. J. Qiao, X. Zhang, C. Liu, L. Lyu, Y. Yang et al., Non-magnetic bimetallic MOF-derived porous carbon-wrapped $\mathrm{TiO}_{2} /$ $\mathrm{ZrTiO}_{4}$ composites for efficient electromagnetic wave absorption. Nano-Micro Lett. 13, 75 (2021). https://doi.org/10.1007/ s40820-021-00606-6

102. S. Gao, G.-S. Wang, L. Guo, S.-H. Yu, Tunable and ultraefficient microwave absorption properties of trace N-doped two-dimensional carbon-based nanocomposites loaded with multi-rare earth oxides. Small 16, 1906668 (2020). https:// doi.org/10.1002/smll.201906668

103. Z. Shen, H. Peng, Z. Xiong, H. Yang, Z. Huang et al., Facile fabrication of $\mathrm{Nd}_{2} \mathrm{O}_{2} \mathrm{~S} / \mathrm{C}$ nanocomposite with enhanced microwave absorption induced by defects. J. Am. Ceram. Soc. (2021). https://doi.org/10.1111/jace.18220

104. Y. Qiu, B. Wen, H. Yang, Y. Lin, Y. Cheng et al., MOFs derived $\mathrm{Co} @ \mathrm{C} @ \mathrm{MnO}$ nanorods with enhanced interfacial polarization for boosting the electromagnetic wave absorption. J. Colloid Interface Sci. 602, 242-250 (2021). https:// doi.org/10.1016/j.jcis.2021.06.006

105. P. Hu, S. Dong, X. Li, J. Chen, X. Zhang et al., A lowcost strategy to synthesize $\mathrm{MnO}$ nanorods anchored on 3D biomass-derived carbon with superior microwave absorption properties. J. Mater. Chem. C 7, 9219-9228 (2019). https:// doi.org/10.1039/c9tc02182e

106. D. Xu, J. Qiao, N. Wu, W. Liu, F. Wang et al., Facile synthesis of three-dimensional porous $\mathrm{Co} / \mathrm{MnO}$ composites derived from bimetal oxides for highly efficient electromagnetic wave absorption. ACS Sustain. Chem. Eng. 7, 8687-8695 (2019). https://doi.org/10.1021/acssuschemeng.9b00529

107. X. Chen, Z. Jia, A. Feng, B. Wang, X. Tong et al., Hierarchical $\mathrm{Fe}_{3} \mathrm{O}_{4} @$ carbon@ $\mathrm{MnO}_{2}$ hybrid for electromagnetic wave absorber. J. Colloid Interface Sci. 553, 465-474 (2019). https://doi.org/10.1016/j.jcis.2019.06.058

108. H. Yang, Z. Shen, H. Peng, Z. Xiong, C. Liu et al., 1D-3D mixed-dimensional $\mathrm{MnO}_{2} @$ nanoporous carbon composites derived from Mn-metal organic framework with full-band ultra-strong microwave absorption response. Chem. Eng. J. 417, 128087 (2021). https://doi.org/10.1016/j.cej.2020. 128087

109. S.X. Zhang, L. Xu, Z.H. Chen, S.T. Fan, Z.J. Qiu et al., Hierarchical porous carbon derived from green cyclodextrin metal-organic framework and its application in microwave absorption. J. Appl. Polym. Sci. 138, 50849 (2021). https:// doi.org/10.1002/app.50849

110. B. Quan, X. Liang, H. Yi, Y. Chen, J. Xiang et al., Thermal conversion of wheat-like metal organic frameworks to achieve $\mathrm{MgO} /$ carbon composites with tunable morphology and microwave response. J. Mater. Chem. C 6, 11659-11665 (2018). https://doi.org/10.1039/c8tc03628d

111. Q. Lai, L. Zheng, Y. Liang, J. He, J. Zhao et al., Metalorganic-framework-derived $\mathrm{Fe}-\mathrm{N} / \mathrm{C}$ electrocatalyst with five-coordinated $\mathrm{Fe}-\mathrm{N}_{\mathrm{x}}$ sites for advanced oxygen reduction in acid media. ACS Catal. 7, 1655-1663 (2017). https://doi. org/10.1021/acscatal.6b02966

112. T. Zhang, J. Wang, W. Zhang, C. Yang, L. Zhang et al., Amorphous Fe/Mn bimetal-organic frameworks: outer and inner structural designs for efficient arsenic(iii) removal. J. Mater. Chem. A 7, 2845-2854 (2019). https://doi.org/10. 1039/c8ta10394a

113. Y. Liu, Z. Chen, W. Xie, F. Qiu, Y. Zhang et al., Enhanced microwave absorption performance of porous and hollow CoNi@C microspheres with controlled component and morphology. J. Alloys Compd. 809, 151837 (2019). https://doi. org/10.1016/j.jallcom.2019.151837

114. L. Wang, B. Wen, X. Bai, C. Liu, H. Yang, NiCo alloy/carbon nanorods decorated with carbon nanotubes for microwave absorption. ACS Appl. Nano Mater. 2, 7827-7838 (2019). https://doi.org/10.1021/acsanm.9b01842

115. T. Zhu, Y. Sun, Y. Wang, H. Xing, Y. Zong et al., Controllable synthesis of MOF-derived $\mathrm{Fe}_{\mathrm{x}} \mathrm{Ni}_{1-\mathrm{x}} @ \mathrm{C}$ composites with dielectric-magnetic synergy toward optimized impedance matching and outstanding microwave absorption. J. Mater. Sci. 56, 592-606 (2021). https://doi.org/10.1007/ s10853-020-05307-w

116. J. Ouyang, Z. He, Y. Zhang, H. Yang, Q. Zhao, Trimetallic FeCoNi@C nanocomposite hollow spheres derived from metal-organic frameworks with superior electromagnetic 
wave absorption ability. ACS Appl. Mater. Interfaces 11, 39304-39314 (2019). https://doi.org/10.1021/acsami.9b114 30

117. W. Liu, J. Pan, G. Ji, X. Liang, Y. Cheng et al., Switching the electromagnetic properties of multicomponent porous carbon materials derived from bimetallic metal-organic frameworks: effect of composition. Dalton Trans. 46, 3700-3709 (2017). https://doi.org/10.1039/c7dt00156h

118. W. Feng, Y. Wang, J. Chen, B. Li, L. Guo et al., Metal organic framework-derived CoZn alloy/N-doped porous carbon nanocomposites: tunable surface area and electromagnetic wave absorption properties. J. Mater. Chem. C 6, 10-18 (2018). https://doi.org/10.1039/c7tc03784h

119. Q. Liao, M. He, Y. Zhou, S. Nie, Y. Wang et al., Highly cuboid-shaped heterobimetallic metal-organic frameworks derived from porous $\mathrm{Co} / \mathrm{ZnO} / \mathrm{C}$ microrods with improved electromagnetic wave absorption capabilities. ACS Appl. Mater. Interfaces 10, 29136-29144 (2018). https://doi.org/ 10.1021/acsami.8b09093

120. J. Pan, W. Xia, X. Sun, T. Wang, J. Li et al., Improvement of interfacial polarization and impedance matching for twodimensional leaf-like bimetallic (Co, $\mathrm{Zn}$ ) doped porous carbon nanocomposites with broadband microwave absorption. Appl. Surf. Sci. 512, 144894 (2020). https://doi.org/10. 1016/j.apsusc.2019.144894

121. B. Wen, H. Yang, Y. Lin, Y. Qiu, Y. Cheng et al., Novel bimetallic MOF derived hierarchical Co@C composites modified with carbon nanotubes and its excellent electromagnetic wave absorption properties. J. Colloid Interface Sci. 605, 657-666 (2022). https://doi.org/10.1016/j.jcis.2021.07.118

122. L. Wang, B. Wen, H. Yang, Y. Qiu, N. He, Hierarchical nestlike structure of $\mathrm{Co} / \mathrm{Fe} \mathrm{MOF}$ derived $\mathrm{CoFe} @ \mathrm{C}$ composite as wide-bandwidth microwave absorber. Compos. Part A Appl. Sci. Manuf. 135, 105958 (2020). https://doi.org/10.1016/j. compositesa.2020.105958

123. X. Liang, Z. Man, B. Quan, J. Zheng, W. Gu et al., Environment-stable $\mathrm{Co}_{\mathrm{x}} \mathrm{Ni}_{\mathrm{y}}$ encapsulation in stacked porous carbon nanosheets for enhanced microwave absorption. Nano-Micro Lett. 12, 102 (2020). https://doi.org/10.1007/ s40820-020-00432-2

124. L. Zhang, P. Yin, J. Wang, X. Feng, J. Dai, Low-frequency microwave absorption of MOF-derived $\mathrm{Co} / \mathrm{CoO} / \mathrm{SrCO}_{3} @ \mathrm{C}$ composites. Mater. Chem. Phys. 264, 124457 (2021). https:// doi.org/10.1016/j.matchemphys.2021.124457

125. H. Wang, L. Xiang, W. Wei, J. An, J. He et al., Efficient and lightweight electromagnetic wave absorber derived from metal organic framework-encapsulated cobalt nanoparticles. ACS Appl. Mater. Interfaces 9, 42102-42110 (2017). https:// doi.org/10.1021/acsami.7b13796

126. L. Huang, C. Chen, X. Huang, S. Ruan, Y.-J. Zeng, Enhanced electromagnetic absorbing performance of MOF-derived Ni/NiO/Cu@C composites. Compos. B Eng. 164, 583-589 (2019). https://doi.org/10.1016/j.compositesb.2019.01.081

127. X. Zhang, J. Qiao, J. Zhao, D. Xu, F. Wang et al., High-efficiency electromagnetic wave absorption of cobalt-decorated $\mathrm{NH}_{2}$-UIO-66-derived porous $\mathrm{ZrO}_{2} / \mathrm{C}$. ACS Appl. Mater.
Interfaces 11, 35959-35968 (2019). https://doi.org/10.1021/ acsami.9b10168

128. X. Zhang, G. Ji, W. Liu, B. Quan, X. Liang et al., Thermal conversion of an $\mathrm{Fe}_{3} \mathrm{O}_{4} @$ metal-organic framework: a new method for an efficient $\mathrm{Fe}-\mathrm{Co} /$ nanoporous carbon microwave absorbing material. Nanoscale 7, 12932-12942 (2015). https://doi.org/10.1039/c5nr03176a

129. J. Yu, J. Yu, T. Ying, X. Liu, X. Zhang et al., Zeolitic imidazolate framework derived $\mathrm{Fe}-\mathrm{N} / \mathrm{C}$ for efficient microwave absorbers. J. Alloys Compd. 838, 155629 (2020). https://doi. org/10.1016/j.jallcom.2020.155629

130. J. Yan, Y. Huang, Y. Yan, X. Zhao, P. Liu, The composition design of MOF-derived Co-Fe bimetallic autocatalysis carbon nanotubes with controllable electromagnetic properties. Compos. Part A Appl. Sci. Manuf. 139, 106107 (2020). https://doi.org/10.1016/j.compositesa.2020.106107

131. H. Zhao, Y. Cheng, J. Ma, Y. Zhang, G. Ji et al., A sustainable route from biomass cotton to construct lightweight and high-performance microwave absorber. Chem. Eng. J. 339, 432-441 (2018). https://doi.org/10.1016/j.cej.2018.01.151

132. M. Yang, Y. Yuan, Y. Li, X. Sun, S. Wang et al., Dramatically enhanced electromagnetic wave absorption of hierarchical $\mathrm{CNT} / \mathrm{Co} / \mathrm{C}$ fiber derived from cotton and metal-organicframework. Carbon 161, 517-527 (2020). https://doi.org/10. 1016/j.carbon.2020.01.073

133. K. Zhang, A. Xie, M. Sun, W. Jiang, F. Wu et al., Electromagnetic dissipation on the surface of metal organic framework (MOF)/reduced graphene oxide (RGO) hybrids. Mater. Chem. Phys. 199, 340-347 (2017). https://doi.org/10.1016/j. matchemphys.2017.07.026

134. S. Lu, Y. Meng, H. Wang, F. Wang, J. Yuan et al., Great enhancement of electromagnetic wave absorption of MWCNTs@ carbonaceous CoO composites derived from MWCNTs-interconnected zeolitic imidazole framework. Appl. Surf. Sci. 481, 99-107 (2019). https://doi.org/10.1016/j. apsusc.2019.03.018

135. J. Fang, Y. Ma, Z. Zhang, B. Yang, Y. Li et al., Metal-organic framework-derived carbon/carbon nanotubes mediate impedance matching for strong microwave absorption at fairly low temperatures. ACS Appl. Mater. Interfaces 13, 33496-33504 (2021). https://doi.org/10.1021/acsami.1c07792

136. K. Zhang, F. Wu, A. Xie, M. Sun, W. Dong, In situ stringing of metal organic frameworks by $\mathrm{SiC}$ nanowires for high-performance electromagnetic radiation elimination. ACS Appl. Mater. Interfaces 9, 33041-33048 (2017). https://doi.org/10. 1021/acsami.7b11592

137. B. Quan, X. Liang, X. Zhang, G. Xu, G. Ji et al., Functionalized carbon nanofibers enabling stable and flexible absorbers with effective microwave response at low thickness. ACS Appl. Mater. Interfaces 10, 41535-41543 (2018). https://doi. org/10.1021/acsami.8b16088

138. X. Xu, F. Ran, H. Lai, Z. Cheng, T. Lv et al., In situ confined bimetallic metal-organic framework derived nanostructure within 3D interconnected bamboo-like carbon nanotube networks for boosting electromagnetic wave absorbing 
performances. ACS Appl. Mater. Interfaces 11, 35999-36009 (2019). https://doi.org/10.1021/acsami.9b14754

139. L. Wang, X. Jia, Y. Li, F. Yang, L. Zhang et al., Synthesis and microwave absorption property of flexible magnetic film based on graphene oxide/carbon nanotubes and $\mathrm{Fe}_{3} \mathrm{O}_{4}$ nanoparticles. J. Mater. Chem. A 2, 14940-14946 (2014). https:// doi.org/10.1039/c4ta02815e

140. A. Nazir, H. Yu, L. Wang, M. Haroon, R.S. Ullah et al., Recent progress in the modification of carbon materials and their application in composites for electromagnetic interference shielding. J. Mater. Sci. 53, 8699-8719 (2018). https:// doi.org/10.1007/s10853-018-2122-X

141. A. Nazir, H. Yu, L. Wang, Y. He, Q. Chen et al., Preparation and properties of ferrocene-based polyfuran/carbon material composites for electromagnetic interference shielding. J. Electron. Mater. 49, 5647-5656 (2020). https://doi.org/10. 1007/s11664-020-08314-4

142. L. Lyu, S. Zheng, F. Wang, Y. Liu, J. Liu, High-performance microwave absorption of MOF-derived $\mathrm{Co}_{3} \mathrm{O}_{4} @ \mathrm{~N}$-doped carbon anchored on carbon foam. J. Colloid Interface Sci. 602, 197-206 (2021). https://doi.org/10.1016/j.jcis.2021.05.184

143. J. Zhang, Z. Yan, X. Liu, Y. Zhang, H. Zou et al., Conductive skeleton-heterostructure composites based on chrome shavings for enhanced electromagnetic interference shielding. ACS Appl. Mater. Interfaces 12, 53076-53087 (2020). https://doi.org/10.1021/acsami.0c14300

144. X. Sun, M. Yang, S. Yang, S. Wang, W. Yin et al., Ultrabroad band microwave absorption of carbonized waxberry with hierarchical structure. Small 15, 1902974 (2019). https:// doi.org/10.1002/smll.201902974

145. S. Wang, Q. Li, K. Hu, S. Wang, Q. Liu et al., A facile synthesis of bare biomass derived holey carbon absorbent for microwave absorption. Appl. Surf. Sci. 544, 148891 (2021). https://doi.org/10.1016/j.apsusc.2020.148891

146. Y. Zhou, W. Zhou, C. Ni, S. Yan, L. Yu et al., "Tree blossom" Ni/NC/C composites as high-efficiency microwave absorbents. Chem. Eng. J. 430, 132621 (2022). https://doi. org/10.1016/j.cej.2021.132621

147. C. Ji, Y. Liu, J. Xu, Y. Li, Y. Shang et al., Enhanced microwave absorption properties of biomass-derived carbon decorated with transition metal alloy at improved graphitization degree. J. Alloys Compd. 890, 161834 (2022). https://doi.org/ 10.1016/j.jallcom.2021.161834

148. X. Qiu, L. Wang, H. Zhu, Y. Guan, Q. Zhang, Lightweight and efficient microwave absorbing materials based on walnut shell-derived nano-porous carbon. Nanoscale 9, 7408-7418 (2017). https://doi.org/10.1039/c7nr02628e

149. Z. Wu, K. Tian, T. Huang, W. Hu, F. Xie et al., Hierarchically porous carbons derived from biomasses with excellent microwave absorption performance. ACS Appl. Mater. Interfaces 10, 11108-11115 (2018). https://doi.org/10.1021/acsami. $7 \mathrm{~b} 17264$

150. Y. Fei, M. Liang, T. Zhou, Y. Chen, H. Zou, Unique carbon nanofiber@Co/C aerogel derived bacterial cellulose embedded zeolitic imidazolate frameworks for high-performance electromagnetic interference shielding. Carbon 167, 575-584 (2020). https://doi.org/10.1016/j.carbon.2020.06.013

151. F. Shahzad, M. Alhabeb, C.B. Hatter, B. Anasori, S.M. Hong et al., Electromagnetic interference shielding with 2D transition metal carbides (MXenes). Science 353, 1137-1140 (2016). https://doi.org/10.1126/science.aag2421

152. X. Li, W. You, C. Xu, L. Wang, L. Yang et al., 3D seed-germination-like MXene with in situ growing CNTs/Ni heterojunction for enhanced microwave absorption via polarization and magnetization. Nano-Micro Lett. 13, 157 (2021). https:// doi.org/10.1007/s40820-021-00680-w

153. M. Yuan, M. Zhou, H. Fu, Synergistic microstructure of sandwich-like $\mathrm{NiFe}_{2} \mathrm{O}_{4} @ \mathrm{SiO}_{2} @ \mathrm{MXene}$ nanocomposites for enhancement of microwave absorption in the whole Ku-band. Compos. Part B-Eng. 224, 109178 (2021). https://doi.org/10. 1016/j.compositesb.2021.109178

154. X. Zhang, H. Wang, R. Hu, C. Huang, W. Zhong et al., Novel solvothermal preparation and enhanced microwave absorption properties of $\mathrm{Ti}_{3} \mathrm{C}_{2} \mathrm{~T}_{\mathrm{x}}$ MXene modified by in situ coated $\mathrm{Fe}_{3} \mathrm{O}_{4}$ nanoparticles. Appl. Surf. Sci. 484, 383-391 (2019). https://doi.org/10.1016/j.apsusc.2019.03.264

155. X. Han, Y. Huang, L. Ding, Y. Song, T. Li et al., $\mathrm{Ti}_{3} \mathrm{C}_{2} \mathrm{~T}_{\mathrm{x}}$ MXene nanosheet metal-organic framework composites for microwave absorption. ACS Appl. Nano Mater. 4, 691-701 (2021). https://doi.org/10.1021/acsanm.0c02983

156. B. Deng, Z. Xiang, J. Xiong, Z. Liu, L. Yu et al., Sandwich-Like $\mathrm{Fe}_{\mathrm{TiO}} @ \mathrm{C}$ nanocomposites derived from MXene/Fe-MOFs hybrids for electromagnetic absorption. Nano-Micro Lett. 12, 55 (2020). https://doi.org/10.1007/ s40820-020-0398-2

157. X. Zhu, H. Qiu, P. Chen, G. Chen, W. Min, Graphitic carbon nitride $\left(\mathrm{g}_{-} \mathrm{C}_{3} \mathrm{~N}_{4}\right)$ in situ polymerization to synthesize MOFCo@CNTs as efficient electromagnetic microwave absorption materials. Carbon 176, 530-539 (2021). https://doi.org/10. 1016/j.carbon.2021.02.044

158. H. Jin, J. Wang, S. Yang, ZIF-67-derived micron-sized cobalt-doped porous carbon-based microwave absorbers with g- $\mathrm{C}_{3} \mathrm{~N}_{4}$ as template. Ceram. Int. 47, 11506-11513 (2021). https://doi.org/10.1016/j.ceramint.2020.12.278

159. L. Yan, X. Wang, S. Zhao, Y. Li, Z. Gao et al., Highly efficient microwave absorption of magnetic nanospindle-conductive polymer hybrids by molecular layer deposition. ACS Appl. Mater. Interfaces 9, 11116-11125 (2017). https://doi. org/10.1021/acsami.6b16864

160. A. Nazir, H. Yu, L. Wang, J. Liu, S. Li et al., Electromagnetic interference shielding effectiveness of ferrocene-based polyimidazole/carbon material composites. Polym. Compos. 41, 2068-2081 (2020). https://doi.org/10.1002/pc.25521

161. X. Sun, X. Lv, M. Sui, X. Weng, X. Li et al., Decorating MOF-derived nanoporous $\mathrm{Co} / \mathrm{C}$ in chain-like polypyrrole (PPy) aerogel: a lightweight material with excellent electromagnetic absorption. Materials 11, 781 (2018). https://doi. org/10.3390/ma11050781

162. J. Luo, K. Zhang, M. Cheng, M. Gu, X. Sun, $\mathrm{MoS}_{2}$ spheres decorated on hollow porous $\mathrm{ZnO}$ microspheres with strong 
wideband microwave absorption. Chem. Eng. J. 380, 122625 (2020). https://doi.org/10.1016/j.cej.2019.122625

163. S. Wang, D. Li, Y. Zhou, L. Jiang, Hierarchical $\mathrm{Ti}_{3} \mathrm{C}_{2} \mathrm{~T}_{\mathrm{x}}$ MXene/Ni Chain/ZnO array hybrid nanostructures on cotton fabric for durable self-cleaning and enhanced microwave absorption. ACS Nano 14, 8634-8645 (2020). https://doi.org/ 10.1021/acsnano.0c03013

164. C. Xu, L. Wang, X. Li, X. Qian, Z. Wu et al., Hierarchical magnetic network constructed by CoFe nanoparticles suspended within "tubes on rods" matrix toward enhanced microwave absorption. Nano-Micro Lett. 13, 47 (2021). https://doi.org/10.1007/s40820-020-00572-5

165. X. Zhang, Z. Wang, L. Xu, K. Zuraiqi, T. Daeneke et al., Liquid metal derived MOF functionalized nanoarrays with ultra-wideband electromagnetic absorption. J. Colloid Interface Sci. 606, 1852-1865 (2022). https://doi.org/10.1016/j. jcis.2021.08.143

166. H. Wang, X. Sun, S. Yang, P. Zhao, X. Zhang et al., 3D ultralight hollow NiCo compound@MXene composites for tunable and high-efficient microwave absorption. Nano-Micro Lett. 13, 206 (2021). https://doi.org/10.1007/ s40820-021-00727-y

167. L. Wang, X. Li, X. Shi, M. Huang, X. Li et al., Recent progress of microwave absorption microspheres by magneticdielectric synergy. Nanoscale 13, 2136-2156 (2021). https:// doi.org/10.1039/d0nr06267g

168. M. Huang, L. Wang, W. You, R. Che, Single zinc atoms anchored on MOF-derived N-doped carbon shell cooperated with magnetic core as an ultrawideband microwave absorber. Small 17, 2101416 (2021). https://doi.org/10.1002/smll. 202101416

169. W. Feng, Y. Wang, Y. Zou, J. Chen, D. Jia et al., ZnO@Ndoped porous carbon $/ \mathrm{Co}_{3} \mathrm{ZnC}$ core-shell heterostructures with enhanced electromagnetic wave attenuation ability. Chem. Eng. J. 342, 364-371 (2018). https://doi.org/10.1016/j. cej.2018.02.078

170. F. Wang, N. Wang, X. Han, D. Liu, Y. Wang et al., Core-shell FeCo@carbon nanoparticles encapsulated in polydopaminederived carbon nanocages for efficient microwave absorption.
Carbon 145, 701-711 (2019). https://doi.org/10.1016/j.carbon.2019.01.082

171. S. Wang, S. Peng, S. Zhong, W. Jiang, Construction of $\mathrm{SnO}_{2} /$ $\mathrm{Co}_{3} \mathrm{Sn}_{2} @ \mathrm{C}$ and $\mathrm{SnO}_{2} / \mathrm{Co}_{3} \mathrm{Sn}_{2} @$ Air@C hierarchical heterostructures for efficient electromagnetic wave absorption. J. Mater. Chem. C 6, 9465-9474 (2018). https://doi.org/10. 1039/c8tc03260b

172. P. Liu, S. Gao, Y. Wang, Y. Huang, W. He et al., Carbon nanocages with $\mathrm{N}$-doped carbon inner shell and $\mathrm{Co} / \mathrm{N}$-doped carbon outer shell as electromagnetic wave absorption materials. Chem. Eng. J. 381, 122653 (2020). https://doi.org/10. 1016/j.cej.2019.122653

173. G. Liu, C. Wu, H. Lei, H. Xin, X. Zhang et al., Anisotropy engineering of metal organic framework derivatives for effective electromagnetic wave absorption. Carbon 181, 48-57 (2021). https://doi.org/10.1016/j.carbon.2021.05.015

174. Z. Gao, Y. Song, S. Zhang, D. Lan, Z. Zhao et al., Electromagnetic absorbers with Schottky contacts derived from interfacial ligand exchanging metal-organic frameworks. J. Colloid Interface Sci. 600, 288-298 (2021). https://doi.org/ 10.1016/j.jcis.2021.05.009

175. J. Xi, E. Zhou, Y. Liu, W. Gao, J. Ying et al., Wood-based straightway channel structure for high performance microwave absorption. Carbon 124, 492-498 (2017). https://doi. org/10.1016/j.carbon.2017.07.088

176. H. Xu, X. Yin, M. Zhu, M. Li, H. Zhang et al., Constructing hollow graphene nano-spheres confined in porous amorphous carbon particles for achieving full $\mathrm{X}$ band microwave absorption. Carbon 142, 346-353 (2019). https://doi.org/10.1016/j. carbon.2018.10.056

177. J. Liu, H. Liang, H. Wu, Hierarchical flower-like $\mathrm{Fe}_{3} \mathrm{O}_{4} / \mathrm{MoS}_{2}$ composites for selective broadband electromagnetic wave absorption performance. Compos. Part A Appl. Sci. Manuf. 130, 105760 (2020). https://doi.org/10.1016/j.compositesa. 2019.105760

178. Z. Zhao, K. Kou, L. Zhang, H. Wu, Optimal particle distribution induced interfacial polarization in bouquet-like hierarchical composites for electromagnetic wave absorption. Carbon 186, 323-332 (2022). https://doi.org/10.1016/j.carbon.2021.10.052 